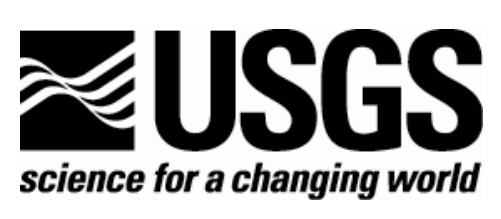

\title{
Wave Data Processing Toolbox Manual
}

By Charlene Sullivan', John Warner', Marinna Martin', Frances Lightsom', George Voulgaris², and Paul Work ${ }^{3}$

IUSGS, Woods Hole Science Center, Woods Hole, MA

2Dept. of Geological Sciences, University of South Carolina, Columbia, SC

${ }^{3}$ Georgia Institute of Technology, School of Civil and Environmental Engineering, Savannah Campus

\section{Open-File Report 2005-1211}

\section{6}

\author{
U.S. Department of the Interior \\ U.S. Geological Survey
}




\title{
U.S. Department of the Interior \\ Gale A. Norton, Secretary
}

\section{U.S. Geological Survey \\ P. Patrick Leahy, Acting Director}

\section{U.S. Geological Survey, Reston, Virginia}

\author{
Contact: \\ John Warner \\ U.S. Geological Survey \\ 384 Woods Hole Road \\ Woods Hole, MA 02543 \\ jcwarner@usgs.gov \\ Telephone: $508-457-237$ or $508-548-8700$
}

For product and ordering information:

World Wide Web: http://www.usgs.gov/pubprod

Telephone: 1-888-ASK-USGS

\author{
For more information on the USGS - the Federal source for science about the Earth, \\ its natural and living resources, natural hazards, and the environment: \\ World Wide Web: http://www.usgs.gov \\ Telephone: 1-888-ASK-USGS \\ Although this report is in the public domain, permission must be secured from the individual \\ copyright owners to reproduce any copyrighted material contained within this report.
}




\section{Contents}

1. Introduction

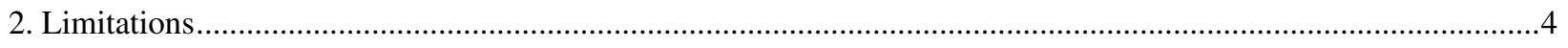

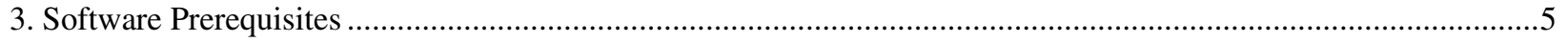

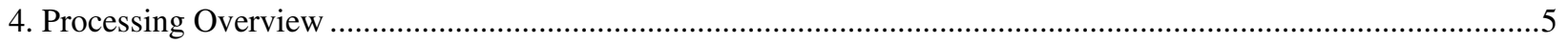

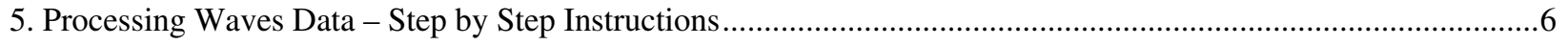

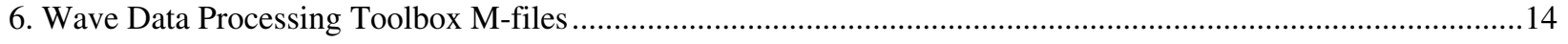

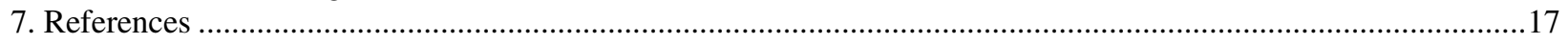

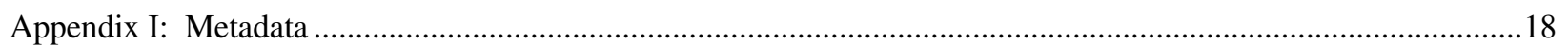

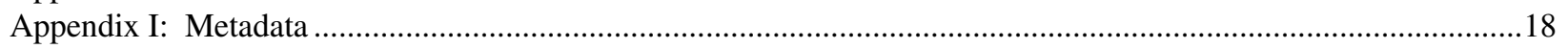

Appendix II: Description of variables in the burst NetCDF file ...............................................................

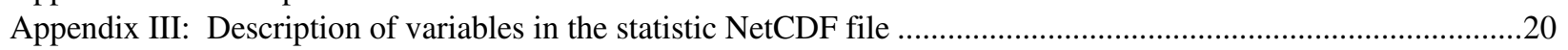

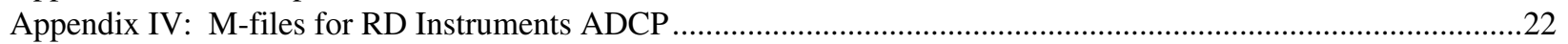




\title{
Wave Data Processing Toolbox Manual
}

\author{
By Charlene Sullivan, John Warner, Marinna Martini, Frances Lightsom, George Voulgaris, and Paul Work
}

\section{Introduction}

Researchers routinely deploy oceanographic equipment in estuaries, coastal nearshore environments, and shelf settings. These deployments usually include tripod-mounted instruments to measure a suite of physical parameters such as currents, waves, and pressure. Instruments such as the RD Instruments Acoustic Doppler Current Profiler (ADCP ${ }^{\mathrm{TM}}$ ), the Sontek Argonaut, and the Nortek Aquadopp ${ }^{\mathrm{TM}}$ Profiler (AP) can measure these parameters. The data from these instruments must be processed using proprietary software unique to each instrument to convert measurements to real physical values. These processed files are then available for dissemination and scientific evaluation. For example, the proprietary processing program used to process data from the RD Instruments ADCP for wave information is called WavesMon. Depending on the length of the deployment, WavesMon will typically produce thousands of processed data files. These files are difficult to archive and further analysis of the data becomes cumbersome. More imperative is that these files alone do not include sufficient information pertinent to that deployment (metadata), which could hinder future scientific interpretation.

This open-file report describes a toolbox developed to compile, archive, and disseminate the processed wave measurement data from an RD Instruments ADCP, a Sontek Argonaut, or a Nortek AP. This toolbox will be referred to as the Wave Data Processing Toolbox. The Wave Data Processing Toolbox congregates the processed files output from the proprietary software into two NetCDF files: one file contains the statistics of the burst data and the other file contains the raw burst data (additional details described below). One important advantage of this toolbox is that it converts the data into NetCDF format. Data in NetCDF format is easy to disseminate, is portable to any computer platform, and is viewable with public-domain freely-available software. Another important advantage is that a metadata structure is embedded with the data to document pertinent information regarding the deployment and the parameters used to process the data. Using this format ensures that the relevant information about how the data was collected and converted to physical units is maintained with the actual data. EPIC-standard variable names have been utilized where appropriate. These standards, developed by the NOAA Pacific Marine Environmental Laboratory (PMEL) (http://www.pmel.noaa.gov/epic/), provide a universal vernacular allowing researchers to share data without translation.

\section{Limitations}

- This Wave Data Processing Toolbox has been developed specifically for use with wave data from:

o RD Instruments Workhorse ADCP

o Sontek Argonaut

o Nortek Aquadopp AP

- The user must provide metadata information in an ASCII-file with specific formatting (templates provided, see discussion below). This information is read from the ASCII file and written to the NetCDF files.

- Wave information must be output from the instrument's proprietary software in ASCII format (details described below).

- For the RD Instruments ADCP, the Wave Data Processing Toolbox assumes the instrument is equipped with the optional waves acquisition firmware.

- For the Sontek Argonaut, the Wave Data Processing Toolbox assumes the wave spectra collection package, Sontek SonWave, is installed for collection of wave frequency spectra data. The user must also specify the LONG data format and metric units during deployment setup for data output. The Wave Data Processing Toolbox does not include provisions for reading the SHORT data format at this time. 


\section{Software Prerequisites}

It is expected that the user has obtained all the proprietary software and necessary files to process the data from their specific instrument. For example, this would include (but is not limited to) the user installing and running the RD Instruments WavesMon software package to process all wave data from the RD Instruments ADCP. Similarly, the user should install and run the ViewArgonaut software package for the Sontek Argonaut and the AquaPro software for the Nortek AP. This manual provides some suggested guidance on program settings for these proprietary software packages. However, the user is responsible for all processing and should consult the user's manual for their proprietary software to determine settings for their specific deployment.

The Wave Data Processing Toolbox described in this manual consists of a Matlab-based package of m-files written in The Mathworks Inc., Matlab ${ }^{\circledR}$ programming language. We assume users have installed The Mathworks Inc., Matlab® software on their operating system. Users must also have installed on their operating system the USGS NetCDF Toolbox. This toolbox was developed by Dr. Charles R. Denham and is freely available for download from: http://woodshole.er.usgs.gov/staffpages/cdenham/public html/MexCDF/nc4ml5.html or http://mexcdf.sourceforge.net/.

NetCDF is the file-format of choice for the USGS due to its platform independence and relative ease of use. Remote users on any number of computer platforms have the ability to load and visualize NetCDF data with a wide variety of open-source software applications, which are freely-available on the World Wide Web.

For more information on NetCDF users are referred to http://my.unidata.ucar.edu/content/software/netcdf/index.html.

ncBrowse is a utility for visualization of NetCDF data. It is freely-available at http://www.epic.noaa.gov/java/ncBrowse/

The Wave Data Processing Toolbox has been tested exclusively with The Mathworks Inc., Matlab® Release 14, Version 7, Service Pack 3 on a Windows XP operating system.

\section{Processing Overview}

\section{Proprietary Software Waves Processing}

Each instrument measures and records time series of velocity and pressure and processes these time series via spectral analysis to provide estimates of directional and/or non-directional energy density spectra and wave parameters. The wave height provided is Hmo, which is $4 \mathrm{x}$ the square root of the area under the non-directional energy density spectrum. It typically corresponds closely to the significant wave height, which is the average of the highest $1 / 3$ of the waves in a record.

Other reported parameters include the peak period, which is the period at which the energy density is maximized. If applicable, the peak wave direction is computed, which is the direction corresponding to the maximum energy in the directional spectrum. The direction is defined as that from which the wave is coming (nautical convention).

Each instrument manufacturer provides a software program for the user to extract measured wave data and to convert (process) the data to engineering units. For the RD Instruments ADCP this software is called WavesMon. For the Sontek Argonaut the software is called ViewArgonaut, and for the Nortek AP the software is called AquaPro. These programs convert the raw binary data files into a series of (typically ASCII) text files containing the time series of wave energy density spectra and wave parameters such as significant wave height, peak wave period, and peak wave direction.

We assume users are familiar with their instrument's proprietary software and will choose processing options that are specific to their project. However, the Wave Data Processing Toolbox assumes that users have saved all processed data to ASCII files. In this manual we include a detailed outline of the processing options chosen for 
each instrument. However, the actual parameters that a user selects are expected to vary with their particular application.

\section{Matlab ${ }^{\circledR}$ Processing}

The Wave Data Processing Toolbox m-files load the output from the proprietary processing software into Matlab®. The toolbox contains provisions for the removal of out-of-water data collected during instrument deployment and recovery. No additional data quality editing is provided at this stage. The statistical wave parameters are converted into EPIC-standard variables and written to a NetCDF file for distribution and archiving. Additionally, for the RD Instruments ADCP and the Nortek AP, individual bursts of velocity and pressure are written to a NetCDF file for archiving.

\section{Processing Waves Data - Step by Step Instructions}

\subsection{Install the Waves Data Processing System}

The Wave Data Processing Toolbox m-files are packed in the file WVTOOLS.zip, Unpack the contents of this zip file to a directory on your operating system's hard drive. This will place a folder named WVTOOLS on your hard drive. The m-files for this toolbox reside in the sub-folders named RDI, Sontek, Nortek, and AddOns. Then start Matlab® and add the WVTOOLS directories to your Matlab® path.

If, for example, you unpacked the WVTOOLS folder to:

c:IMFILES\WVTOOLS

you should type the following at the Matlab® command prompt to add the WVTOOLS directories to your Matlab® path:

> addpath(genpath('c:\MFILES\WVTOOLS'))

\subsection{Directory Setup}

Users should run the proprietary software for their instrument and the Wave Data Processing Toolbox mfiles in a directory that contains the raw binary data file downloaded from their instrument. If a directory with this file already exists on your operating system's hard drive, we advise you to create a sub-directory and copy the instrument's raw binary file to this sub-directory. This will isolate processing of the waves data from other processing that might occur using the file. As an example below, we create a sub-directory named 'wavesmon' in which we run the WavesMon software package and the Wave Data Processing Toolbox m-files for the RD Instruments ADCP. The raw binary data file from this instrument is copied into this directory from the directory above, '7221wh'. Similarly, we would create sub-directories named 'viewarg' and 'aquapro' for the Sontek Argonaut and Nortek AP, respectively.

Thus: C:17221wh is a directory with raw binary data file downloaded from your instrument

C:17221wh/wavesmon is a subdirectory with a copy of the raw binary data file, a metadata file, processed data output from the instrument's proprietary software, and NetCDF data files that will be output by this Wave Data Processing Toolbox. 


\subsection{The Metadata File}

Users should next create a metadata file and place it in the sub-directory created above in section 5.2. A metadata file is a simple text file containing important information regarding the collection of the data. For example, it will typically include the location, date, and time of data collection. This information is crucial for scientific data interpretation. Common metadata fields are described in Appendix I. The Wave Data Processing Toolbox is designed to read a metadata file that has specific formatting. These files are instrument-specific. We provide example metadata files for each instrument in the Wave Data Processing Toolbox package of $\mathrm{m}$-files. The files include metaRDI.txt for the RD Instruments ADCP, metaNortek.txt for the Nortek Aquadopp AP and metaSontek.txt for the Sontek Argonaut. It is recommended that users copy the included metadata file for their instrument into the subdirectory created in section 5.2 and use it as a template for their metadata file.

Once the metadata file is created, users should run the proprietary software package specific to their instrument in order to obtain ASCII file output of processed wave measurements. Step-by-step instructions for the proprietary software are provided in sections 5.4 for the RD Instruments ADCP, in 5.5 for the Sontek Argonaut and in 5.6 for the Nortek AP. Included in these instructions are suggested processing options (where available) for each instrument.

\subsection{Processing for an RD Instruments ADCP}

The RD Instrument ADCP records three different types of time series from which wave properties may be computed: pressure, range to the surface along each of its four beams (i.e., water level), and orbital velocities of the surface waves taken from three bins nearest the surface in each of the four beams. It is possible to estimate nondirectional wave energy spectra, and thus wave height and period, from any of the three time series, but the velocity time series are required for definition of the directional distribution of the wave energy.

The WavesMon software package processes the raw binary ADCP data file and produces a series of computed data files. Pressure time series are output to files named Pressyyyynnddhhmmssxx.txt, where Press is pressure, and the remaining characters are year (yyyy), month (nn), day(dd), hour (hh), minute (mm), second (ss), and milli-second (xx). Time series of the range to surface are output to files named Strkyyyynnddhhmmssxx.txt, whereas time series of orbital velocities are output to Velyyyynnddhhmmssxx.txt. The orbital velocity measurements are used to calculate directional wave spectra, which are saved in output files called DSpecyynnddhhmm.txt, where Dspec is directional spectra, and the remaining characters are year (yy), month (nn), day (dd), hour (hh), and minute ( $\mathrm{mm}$ ) of the sample average. Data from all three methods are used to compute nondirectional spectra, which are saved in files called PSpecyynnddhhmm.txt, SSpecyynnddhhmm.txt, and VSpecyynnddhhmm.txt where the PSpec* are derived from the pressure measurements, SSpec* are derived from the range to surface measurements, and VSpec* are derived from the orbital velocity measurements. Time series of wave parameters such as significant wave height, peak wave period, and peak wave direction are output in a file called *_LogData.000.

Users are cautioned against re-running WavesMon in a directory in which WavesMon was previously run. Doing so creates an additional time series of wave parameters called *_LogData.001, rather than overwriting the previous time series. The Wave Data Processing Toolbox assumes WavesMon was run once in a directory and that only one time series file called *_LogData.000 exists in the directory. Users are referred to RD Instrument's Waves User's Guide and the RDI Waves Primer for detailed WavesMon processing instructions and information regarding WavesMon's various processing options.

\subsubsection{Start WavesMon}

Double-click the WavesMon icon on your desktop to start WavesMon. Alternatively click the start menu and select all programs then RD Instruments and then WavesMon.

In the WavesMon File menu select New Setup. Then select Playback in the Choose a realtime or playback dialogue and click OK. Click OK again if a Tip pops up. 
In the Save Setup As dialog browse to the location of the directory you created during 'Directory Setup' in section 5.2. Then enter a filename for your setup file. We use a filename that corresponds to our mooring number and instrument, such as $722 \mathrm{wh}$, where ' 722 ' is the mooring number and 'wh' is for Workhorse ADCP. This file name is added by default to the *_LogData.000 file that contains the time series of wave parameters (i.e.

722wh_LogData.000).

In the Setup Playback screen select Advanced. The Setup Playback (Advanced) screen should now appear.

\subsubsection{Designate Input and Output Options}

In the Setup Playback (Advanced) screen select the Input tab. On the Input screen select Browse, then select your raw binary adcp data file and click Open.

Next, select the Output (fig. 1) tab from the Setup Playback (Advanced) screen. Under Processed Waves Data make sure both Processed Data and Save Processed Data to Text Files are checked (Required). Also make sure the Wave Log Format is 5 (Required).

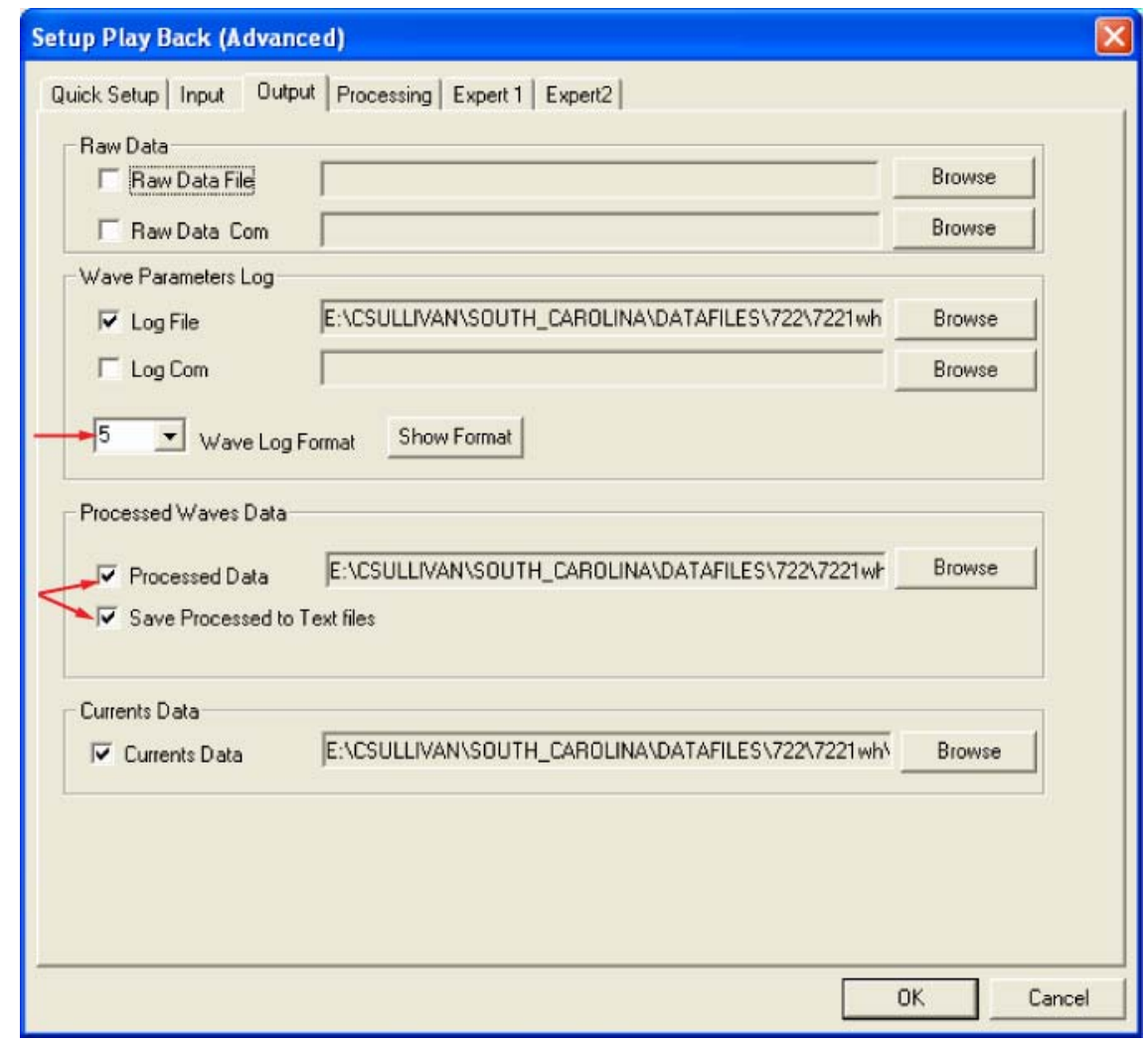

Figure 1: The Output tab from the WavesMon Setup Playback (Advanced) screen. Red arrows identify specific WavesMon processing options described in text.

\subsubsection{Select Processing Options}

Select the Processing (fig. 2) tab from the Setup Playback (Advanced) screen. Under What to Process make sure all boxes are checked. This will process and save the velocity, surface, and pressure time series and spectra, the directional spectra, and the wave parameters (Required). Under How to Process change Lower Freq Threshold from its default value (0.01) to 0.05. Change Upper Freq Threshold for velocity, V, from its default 
value (0.35) to 0.5. Under ADCP Environment check the Transducer Altitude box and enter the transducer's altitude in centimeters. If your pressure sensor was working and was not significantly biofouled, be sure that Pressure Sensor is selected next to Depth From. Otherwise select Surface Track. Under ADCP Heading check the Magnetic Variation box and enter your location's magnetic variation in degrees. Magnetic variation is positive east of true north and negative when west

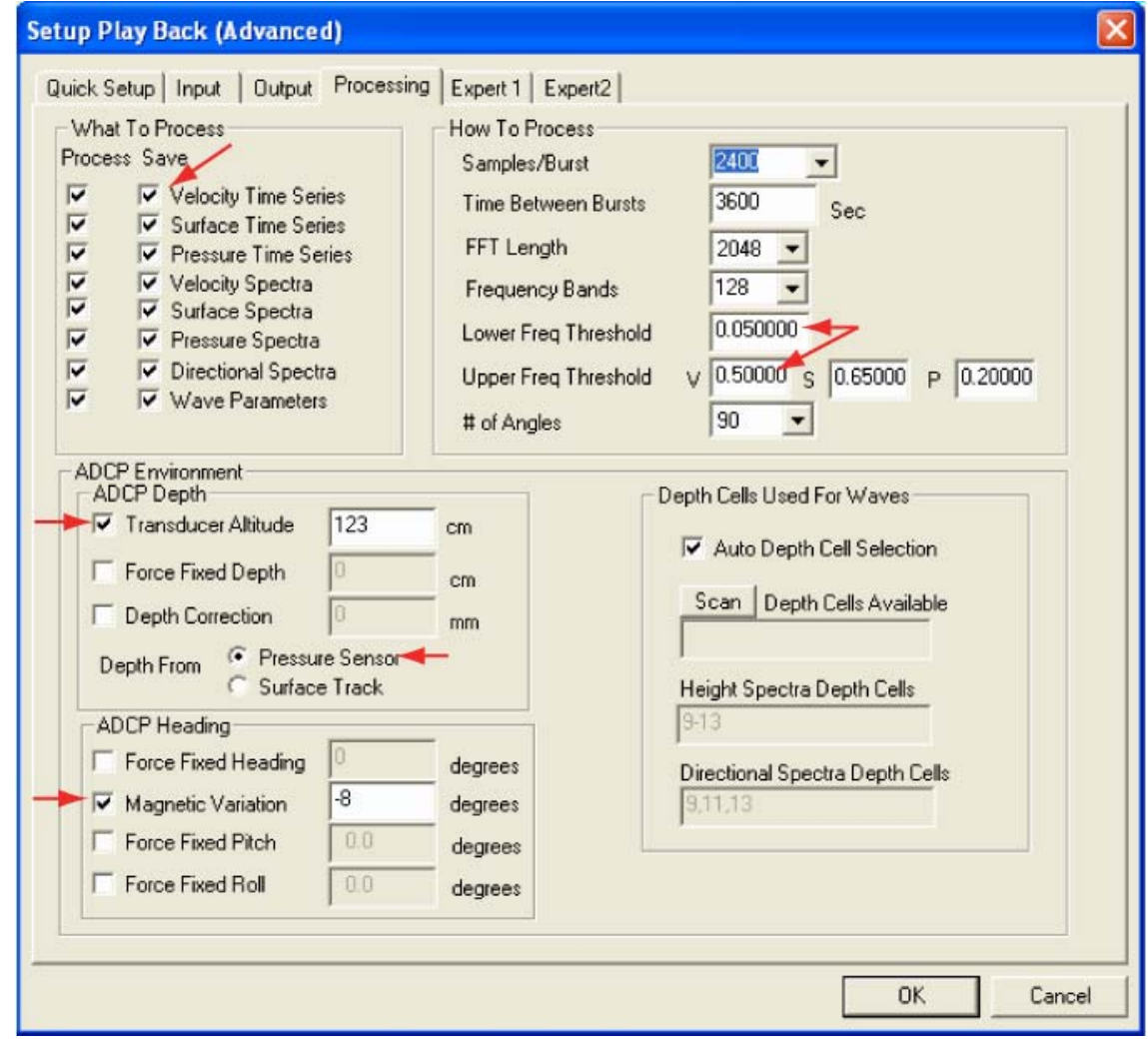

Figure 2: The Processing tab from the WavesMon Setup Playback (Advanced) screen. Red arrows identify specific WavesMon processing options described in text.

Next, select the Expert 1 (fig. 3) from the Setup Playback (Advanced) screen. In the box next to IMLM Iterations click the down arrow to change the value from its default (1) to 3 . Also check the box next to Output Dir Spec in Power x Power (Required). 


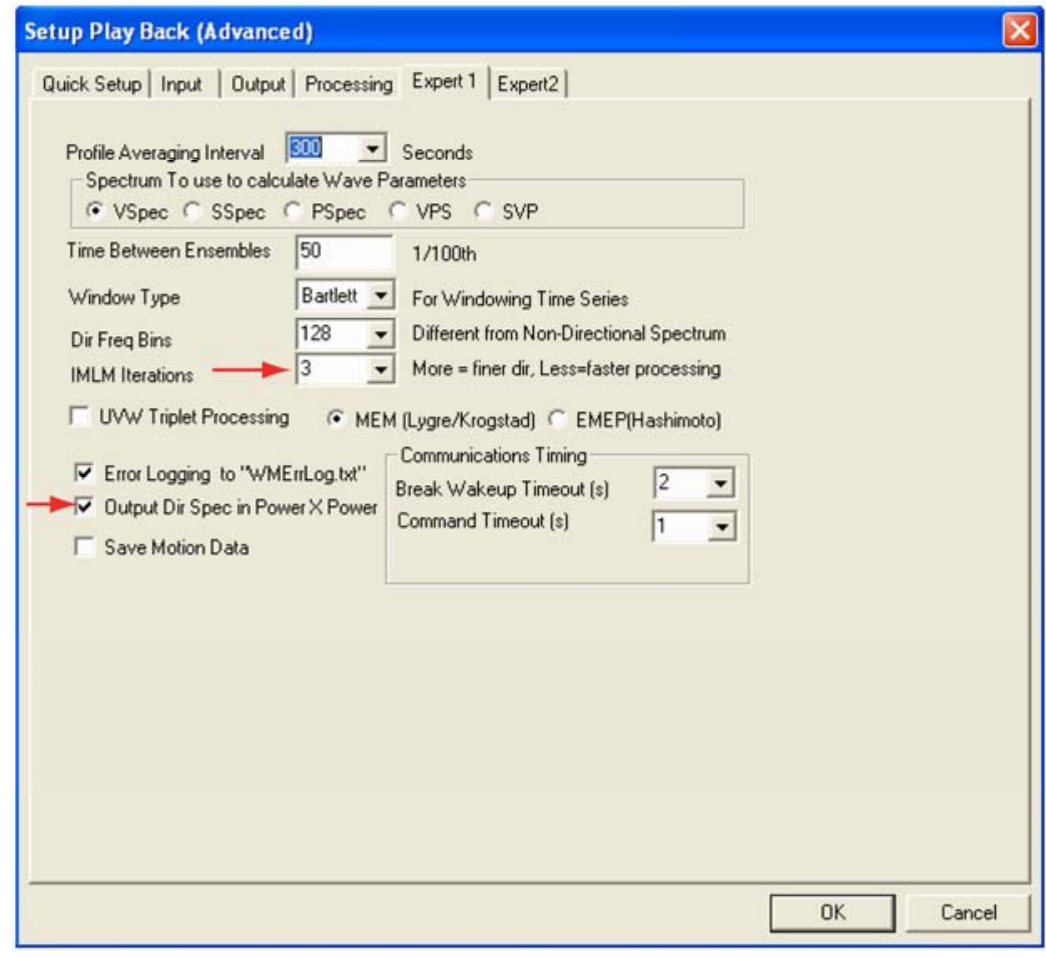

Figue 3: The Expert 1 tab from the WavesMon Setup Playback (Advanced) screen. Red arrows identify specific WavesMon processing options described in text.

Lastly, select the Expert 2 (fig. 4) from the Setup Playback (Advanced) screen. Under Data Screening check the box next to Use File Buffer for Data. Under Misc check the box next to Correct for Currents. Click OK to close the Expert 2 tab. Click OK again to close the Setup Playback (Advanced) screen. 


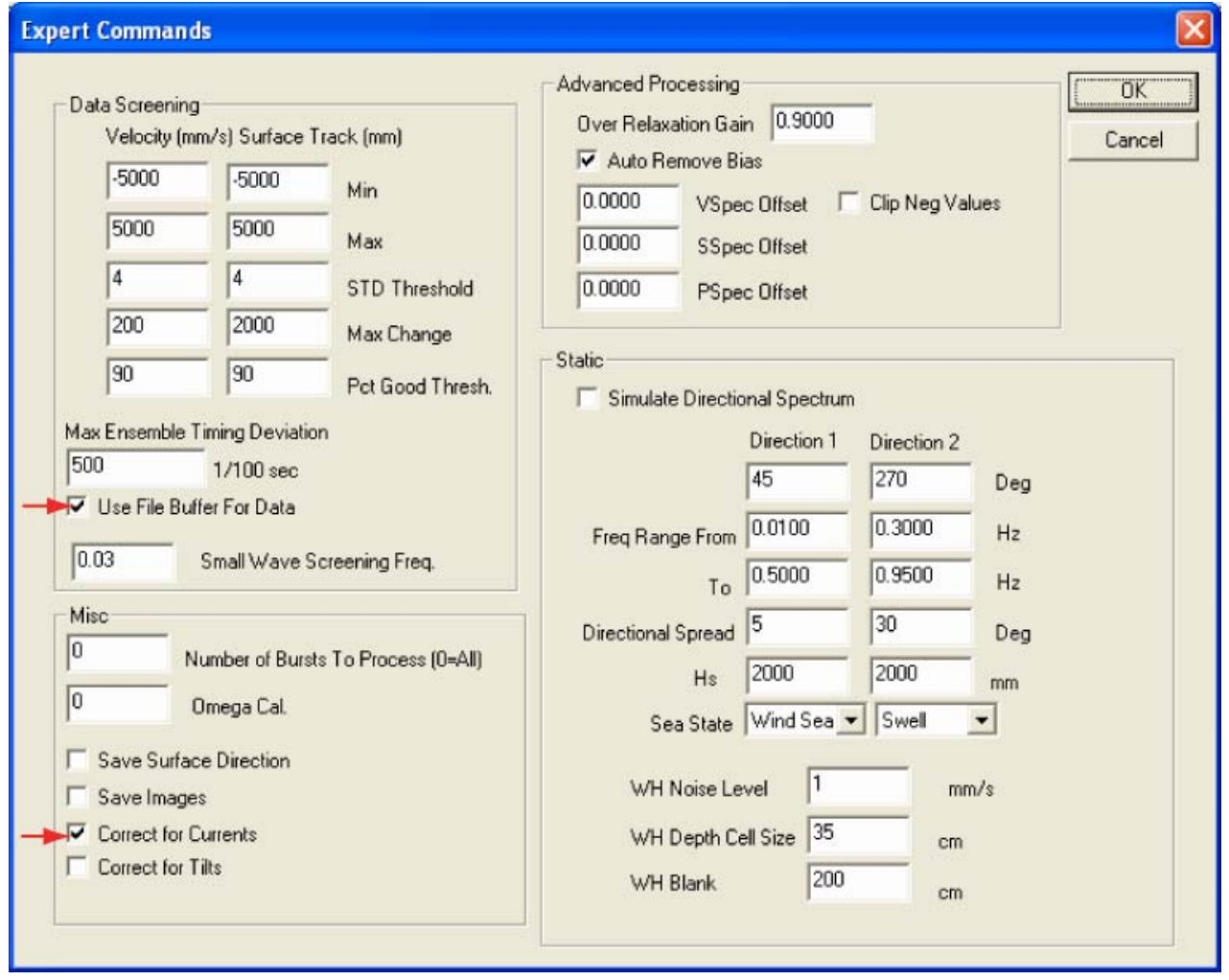

Figure 4: The Expert 2 tab from the WavesMon Setup Playback (Advanced) screen. Red arrows identify specific WavesMon processing options described in text.

\subsubsection{Start WavesMon Processing}

The Setup Playback screen should now be displayed. Select OK on this screen to close it. Select the green Go button on the main WavesMon screen to commence WavesMon processing. This processing produces the Press*.txt, Strk*.txt, Vel*.txt, DSpec*.txt, PSpec*.txt, VSpec*.txt, SSpec*.txt, and *_LogData.000 ASCII output files.

Once WavesMon has completed, the user should exit WavesMon and follow the step-by-step instructions outlined in section 5.7 to run the Waves Data Processing Toolbox.

\subsection{Processing for a Sontek Argonaut}

The Sontek Argonaut measures wave pressure and computes a non-directional wave energy spectra. The Waves Data Processing Toolbox assumes the user has specified the LONG data format with metric units during system configuration. It also assumes the user has the wave spectra collection package, Sontek SonWave, installed for collection of wave frequency spectra data. The software that is proprietary to the Sontek Argonaut is called ViewArgonaut. The pressure signal is used to estimate wave height.

ViewArgonaut is used to convert a raw binary data file (*.arg) from the instrument to ASCII text files. It produces output files called *.ctl, *.snr, *.std, *.vel, and *.dat, where '*' is the name given to your deployment when setting up the ADP for deployment. The file *.ctl is an ASCII file containing information on the system configuration, and the contents of the other data files. The files *.snr, *.std, and *.vel contain multi-cell (profiling) data output for all samples for all cells. The *.vel file includes the $\mathrm{x}$ - and $\mathrm{y}$ - components of mean velocity, and speed and direction of the mean current. The *.std file includes the standard error in $\mathrm{x}$ - and $\mathrm{y}$ - components of mean velocity. The *.snr file contains the signal to noise ratio and amplitude for each beam. The processed wave data is 
written to the *.dat file and includes significant wave height, peak period, and non-directional wave energy spectra information.

\subsubsection{Start ViewArgonaut}

Double-click the ViewArgonaut icon on your desktop to start the application. In the ViewArgonaut menu select Processing.

\subsubsection{Select Input}

Next, select File then Open. In the open dialogue box browse to the directory created in section 5.2.0 that contains the raw binary file from the instrument. Select the file and select Open. A box with Argonaut File information is displayed. Preview the information and select Ok. It is now possible for the user to view different displays of processed data if they choose to do so.

\subsubsection{Select Output}

In order to generate ASCII files with processed data, the user must export processed data from ViewArgonaut. In ViewArgonaut's File menu select Export Data. In the ASCII File Output box under Save Files to Path be sure to save files to the same directory in which the raw binary file from the instrument resides (this will be the directory created in section 5.2). It is important to check this path as the software defaults to the directory specified in the previous ViewArgonaut session. Under Output File Name use the default, which should be the same as your input file name without the .arg file extension. Under Samples make sure All Samples is checked (this is the default).

\subsubsection{Export Data}

Select Export All Variables in the lower right corner of the ASCII File Output box. If a box pops up regarding discharge data click ok to resume exporting data. A box titled Output Ascii File Messages should be displayed. In that box should be three messages stating the configuration data, the time series data, and the multicell data were successfully written. Click OK in this box. Then click Close on the ASCII File Output box.

At this point the user should exit ViewArgonaut and follow the step-by-step instructions outlined in section 5.7 to run the Waves Data Processing Toolbox.

\subsection{Processing for a Nortek AP}

The Nortek AP measures wave pressure and near-bed velocities. The proprietary software for the Nortek AP is called AquaPro and is used to convert binary data files to ASCII text files. The software produces output files called *.a1, *.a2, *.a3, *.hdr, *.sen, *.v1, *.v2, *.v3, *.whd, and *.wad. The files *.v1, *.v2, and *.v3 contain current velocities in user-defined coordinates, which can be as beam coordinates, as orthogonal coordinantes in relation to the sensor head or as geographic coordinates. The files *.a1, *.a2, and *.a3 contain amplitudes along the instrument's three beams. Instrument set-up information is located in the *.hdr file. A summary of each burst is supplied in the file *.wad. Burst-by-burst data is provided in the file *.whd. The file *.sen, contains a summary of sensor information, such as instrument heading, pitch, roll, and battery voltage. The m-files in this Wave Data Processing Toolbox use the PUV method to estimate wave parameters. If current velocities were collected in beam coordinates, they will be converted to geographical coordinates by the toolbox prior to the application of the PUV method. The pressure signal is used to estimate wave height and the measurements of the waves' orbital velocities provide an estimate of the wave direction, after correction is performed for depth attenuation. Additional information is provided at:

\section{http://www.nortek-as.com/technotes/PUVWaves.pdf}




\subsubsection{Start AquaPro}

Double-click the AquaPro icon on your desktop to start the application.

\subsubsection{Select Input}

Under the Deployment menu, select Data Conversion. In the Data Conversion pop-up box, select Add File. Browse to the folder created in section 5.2 that contains the instrument's raw binary data file (*.prf). Select this file and then select Open.

\subsubsection{Select Output}

In order to generate ASCII files using processed data, the user must export processed data from AquaPro. In the Data Conversion box next to Add prefix, enter a character string that will be pre-pended to the output files.

\subsubsection{Export Data}

Select the blue arrow in the center of the Data Conversion box. In the Data Conversion pop-up box, there should be check marks next to the following boxes: Header, Velocity, Amplitude, Sensors, and Wave. Select OK to commence exporting of data. When processing is completed, the output file names will be displayed under Converted files in the Data Conversion box. Select Done and then close the AquaPro software. The *.a1, *.a2, *.a3, *.hdr, *.sen, *.v1, *.v2, *.v3, *.whd, and *.wad should now exist in the directory containing the instrument's raw binary data file.

At this point the user should exit AquaPro and follow the step-by-step instructions outlined in section 5.7 to run the Wave Data Processing Toolbox.

\subsection{Matlab® Processing}

\subsubsection{Start Matlab®}

Double-click the Matlab icon on your desktop to start Matlab®. Alternatively, click the start menu and select all programs then Matlab x.xx, Matlab x.xx where 'x.xx' is the version of Matlab® installed on your computer.

\subsubsection{Change directories}

At the Matlab command prompt change directories to the directory you created in section 5.2. This directory should contain the raw binary data file from the instrument, the metadata file, and the ASCII output files from your instrument's proprietary software. For example, the directory created in section 5.2 contains the raw binary data file from an RD Instruments ADCP, a metadata file, and WavesMon-generated ASCII output files. Type the following at Matlab's command prompt in order to change directories to this directory:

>> cd C: :7221whlwavesmon

\subsubsection{Run the Wave Data Processing Toolbox}

The Wave Data Processing Toolbox is run by a driver $\mathrm{m}$-file that calls a series of $\mathrm{m}$-files (outlined below) to load proprietary software output files, to convert data into EPIC-compatible variables where appropriate, and to 
convert data to NetCDF. These m-files were developed by the authors, but they include calls to other usercontributed $\mathrm{m}$-files. We include all required $\mathrm{m}$-files in the Wave Data Processing Toolbox package.

The driver m-files for the instruments supported by this package are called adcpWvs2nc.m (for the RD Instruments adcp), argnWvs2nc.m (for the Sontek Argonaut), and aqdpWvs2nc.m (for the Nortek AP). The inputs for these driver $m$-files include the metadata file name and a character string that represents the NetCDF file names to which data will be written. To run the Wave Data Processing Toolbox the user would type the following at Matlab's command prompt:

For the RD Instruments ADCP

>> adcpWvs2nc(metaFile, outFileRoot)

For the Sontek Argonaut

> $\operatorname{argnWvs2nc(metaFile,~outFileRoot)~}$

For the Nortek AP

>> aqdpWvs2nc(metaFile, outFileRoot)

The input metaFile is a character string that specifies the name of your metadata file. This should be surrounded by single quotes and specified without the file extension .txt. The input outFileRoot is a character string that specifies the name of the netCDF files to which you would like to write the data. This string should be surrounded by single quotes, and the NetCDF file extension, .nc, is not necessary.

The Wave Data Processing Toolbox consumes a large amount of computer memory when it accumulates data into multi-dimensional arrays. We suggest users have no other programs running on their system while running the toolbox, and use a clean start of Matlab to prevent Matlab out-of-memory errors. Users may also wish to turn their hardware acceleration off before running the toolbox.

For the RD Instruments ADCP, bad data screening and removal are accomplished internally by WavesMon. The entire time series of pressure, velocities, and wave parameters are converted to NetCDF and no user-interaction is required. However, for both the Sontek Argonaut and the Nortek Aquadopp AP, user-interaction is required. The user is asked to view a plot of pressures and velocities (for the Nortek), or wave parameters (for the Sontek), and decide the first and last good bursts of data. All data between the first and last good bursts is converted to NetCDF. This provision was included to exclude out-of-water data, collected during deployment and recovery of the instruments, from the NetCDF files.

The NetCDF files outFileRootr-cal.nc and outFileRootp-cal.nc will be written to the directory you created in section 5.2. The first NetCDF file contains the time series of pressure and velocities. Please note that this NetCDF file is created only for the RD Instruments ADCP and the Nortek AP. We do not create this NetCDF file for the Sontek Argonaut, as the pressure time series from which wave parameters and spectra are calculated is not output by ViewArgonaut. The second NetCDF file contains the statistical wave parameters, and these files are created for all 3 supported instruments. Please refer to Appendix II and Appendix III for a listing of variables in the burst and statistic NetCDF files, respectively. This completes wave data processing with the Wave Data Processing Toolbox.

\section{Wave Data Processing Toolbox M-files}

The m-files called by the Wave Data Processing Toolbox are outlined below. These m-files do NOT require editing. Please report all bugs to John Warner at mailto:hjcwarner@usgs.gov. The authors would like to acknowledge NortekUSA for the inclusion of their m-files, wds.m, hs.m, logavg.m, and wavek.m in this processing package. All of Nortek's m-files were downloaded from http://www.nortekusa.com/principles/Waves.html\#MatlabToolkit. The mfiles hs.m, logavg.m, and wavek.m remain unmodified from their original form. The mfile wds.m contains one modification to prevent erroring in Matlab®. We would also like to acknowledge Dr. Richard P. Signell, who provided the functions julian.m, and gregorian.m. 


\subsection{M-files for RD Instruments ADCP}

M-files called for the RD Instruments ADCP are located in the sub-folder RDI of the WVTOOLS folder that was downloaded and installed in section 5.1.0. The m-files are also provided in this manual in Appendix IV. An outline and a short description of each $\mathrm{m}$-file are provided below.

A. adcpWvs2nc.m Primary driver mfile, controls calls to other m-files

B. nccreate_adcpWvs.m $\sim$ a function to create and define the raw and processed NetCDF files for RD Instruments ADCP wave data storage, archival, and dissemination

C. read_adcpWvs.m $\sim$ a function to load the WavesMon data file *_LogData.000 containing the time series of wave parameters

D. ncwrite_adcpWvs.m $\sim$ a function to write the time series of wave parameters to the processed NetCDF file

E. read_adcpWvs_spec.m a function to load the WavesMon data files Dspec*.txt, Pspec*.txt, Sspec*.txt, and Vspec*.txt containing the time series of directional and non-directional wave energy spectra

F. ncwrite_adcpWvs_spec.m $\sim$ a function to write the time series of directional and non-directional wave energy spectra to the processed NetCDF file

G. read_adcpWvs_raw.m a function to load the WavesMon data files Press*.txt, Strk*.txt, and $\mathrm{Vel}^{*}$.txt containing the raw time series of pressure, range to surface track, and orbital velocity data

H. ncwrite_adcpWvs_raw.m $\sim$ a function to write the raw time series of pressure, range to surface track, and orbital velocity data the raw NetCDF file 


\subsection{M-files for Sontek Argonaut}

M-files called for the Sontek Argonaut are located in the sub-folder Sontek of the WVTOOLS folder that was downloaded and installed in section 5.1. The m-files are also provided in this manual in Appendix V. An outline and a short description of each $\mathrm{m}$-file are provided below.

A. argnWvs2nc.m Primary driver mfile, controls calls to other $\mathrm{m}$ files

B. get_meta_sontek.m a function to load user-defined metadata and instrument setup information

C. nccreate_argnWvs.m a function to create and define the raw and processed NetCDF files for Sontek Argonaut wave data storage, archival, and dissemination

D. read_argnWvs.m a function to load the ViewArgonaut file *.dat containing the time series of wave parameters and non-directional wave energy spectra

E. ncwrite_argnWvs.m a function to write the time series of wave parameters and non-directional wave energy spectra to the processed NetCDF file

\subsection{M-files for Nortek AP}

M-files called for the Nortek AP are located in the sub-folder Nortek of the WVTOOLS folder that was downloaded and installed in section 5.1. The m-files are also provided in this manual in Appendix VI. An outline and a short description of each $\mathrm{m}$-file are provided below.

A. aqdpWvs2nc.m Primary driver mfile, controls calls to other $\mathrm{m}$ files

B. get_meta_nortek.m a function to load user-defined metadata and instrument setup information

C. wad2puv.m a function to read the Nortek Aquadopp AP file *.wad, split the file into individual bursts, and perform PUV analysis

D. nccreate_aqdpWvs.m a function to create and define the raw and processed NetCDF files for Nortek AP wave data storage, archival, and dissemination

E. ncwrite_aqdpWvs.m $\sim$ a function to write the time series of wave parameters and non-directional wave energy spectra to the processed NetCDF file

F. ncwrite_aqdpWvs_raw.m a function to write the time series of raw pressures and velocities to the raw NetCDF file 


\section{References}

RD Instruments Waves Primer: Wave Measurements and the RDI ADCP waves array technique. http://www.rdinstruments.com/waves.html.

SonTek/YSI Argonaut Acoustic Doppler Current Meter Technical Documentation. http://www.sontek.com/product/sw/viewarg/viewargonaut.htm.

Aquadopp Current Meter User manual.

http://www.nortek-as.com/support/manuals/Aquadopp Manual.pdf

PUV Wave Directional Spectra: How PUV Wave Analysis Works.

http://www.nortek-as.com/technotes/PUVWaves.pdf 


\section{Appendix I: Metadata}

The metadata file contains information regarding the instrument and the deployment. Example metadata files with specific formatting are provided in the Wave Data Processing Toolbox for each instrument. See metaRDI.txt, metaNortek.txt, and metaSontek.txt. Users may copy and edit these files to suit their project needs, or create their own metadata files. Users may choose to add or remove metadata fields. However, they must keep in mind that the metadata field's description must start at column 20 and not exceed column 83 of the file. Example metadata fields are described below.

\begin{tabular}{|c|c|c|}
\hline Metadata Field & Field Description & Example \\
\hline Mooring & mooring identification number & '7201' \\
\hline Deployment_date & date of instrument deployment & '28-Oct-2003' \\
\hline Recovery_date & date of instrument recovery & '21-Jan-2004 \\
\hline INST_TYPE & type of instrument and instrument manufacturer & 'RD Instruments ADCP' \\
\hline history & NetCDF file history & $\begin{array}{l}\text { 'ADCP wave data processed with RDI } \\
\text { WavesMon software' }\end{array}$ \\
\hline DATA_SUBTYPE & description of data type & 'MOORED' \\
\hline DATA_ORIGIN & data originating institution & 'USGS/WHSC' \\
\hline COORD_SYSTEM & coordinate system of the data & 'GEOGRAPHIC' \\
\hline WATER_MASS & $\begin{array}{l}\text { water mass flag used for EPIC contouring } \\
\text { programs }\end{array}$ & '?' \\
\hline POS_CONST & consistent position flag ( 1 = not consistent) & ‘ 0 ' \\
\hline DEPTH_CONST & consistent depth flag ( 1 = not consistent $)$ & ${ }^{\prime} 0$ ’ \\
\hline WATER_DEPTH & water depth in meters & '11.3 M' \\
\hline DRIFTER & drifter flag (=1 if drifter) & ' 0 ' \\
\hline VAR_FILL & missing or bad data value identifier & '1.0000000409184788E35' \\
\hline EXPERIMENT & experiment name & 'Myrtle Beach' \\
\hline PROJECT & project name & 'South Carolina Coastal Erosion Study' \\
\hline DESCRIPT & location and/or site of data collection & 'Site 1 ADCP' \\
\hline longitude & instrument location & $-78.7893 ’$ \\
\hline latitude & instrument location & '33.6497' \\
\hline FILL_FLAG & data fill flag (=1 if data has fill values) & '0’ \\
\hline COMPOSITE & number of pieces in composite series & ‘ 0 ' \\
\hline magnetic_variation & $\begin{array}{l}\text { degrees between magnetic and true north at data } \\
\text { location }\end{array}$ & $'-8.22$ ' \\
\hline
\end{tabular}




\section{Appendix II: Description of variables in the burst NetCDF file}

The following tables list a description of the variables output to the burst NetCDF file (outFileRootr-cal.nc) for the RD Instruments ADCP and the Nortek AP. A burst NetCDF file is not created for the Sontek Argonaut. Time is stored in the variables 'time' and 'time2'. The variable 'time' contains the Julian Day where Julian Day 2440000 begins at 0000 hours on May 23, 1968. The variable 'time2' contains milliseconds (msec) for each Julian Day. These two variables can be combined as in (1) to yield the time of each observation in Julian Days.

$$
\text { jday }=\text { time }+(\text { time } 2 / 1000 / 24 / 3600)
$$

\section{For RDI ADCP:}

\begin{tabular}{|l|l|l|}
\hline Variable & Description & Units \\
\hline time & Time in Julian Days & Julian Day \\
\hline time2 & Time in Julian Days & $\mathrm{msec}$ of each Julian Day \\
\hline burst & Burst number & counts \\
\hline lat & Latitude & degree_north \\
\hline lon & Longitude & degree_east \\
\hline sample & Sample number & counts \\
\hline press & Pressure sensor derived depth & $\mathrm{mm}$ \\
\hline strk & Along-beam surface track & $\mathrm{mm}$ \\
\hline vel & Along-beam velocity & $\mathrm{mm} / \mathrm{s}$ \\
\hline
\end{tabular}

\section{For Nortek AP:}

\begin{tabular}{|l|l|l|}
\hline Variable & Description & Units \\
\hline time & Time in Julian Days & Julian Day \\
\hline time2 & Time in Julian Days & msec of each Julian Day \\
\hline burst & Burst number & counts \\
\hline lat & Latitude & degree_north \\
\hline lon & Longitude & degree_east \\
\hline sample & Sample number & counts \\
\hline hght_18 & $\begin{array}{l}\text { Height of the sea surface relative to } \\
\text { sensor }\end{array}$ & $\mathrm{m}$ \\
\hline u_1205 & Eastward velocity & $\mathrm{cm} / \mathrm{s}$ \\
\hline v_1206 & Northward velocity & $\mathrm{cm} / \mathrm{s}$ \\
\hline w_1204 & Vertical velocity & $\mathrm{cm} / \mathrm{s}$ \\
\hline amp & Beam amplitude & $\mathrm{counts}$ \\
\hline
\end{tabular}




\section{Appendix III: Description of variables in the statistic NetCDF file}

The following tables list a description of the variables output to the statistic NetCDF file (outFileRootpcal.nc) for the RD Instruments ADCP, the Nortek AP, and the Sontek Argonaut. Time is stored in the variables 'time' and 'time2'. The variable 'time' contains the Julian Day where Julian Day 2440000 begins at 0000 hours on May 23, 1968. The variable 'time2' contains milliseconds (msec) for each Julian Day. These two variables can be combined as in (1) above to yield the time of each observation in Julian Days.

\section{For the RDI ADCP:}

\begin{tabular}{|c|c|c|}
\hline Variable & Description & Units \\
\hline time & Time in Julian Days & Julian Day \\
\hline time2 & Time in Julian Days & msec of each Julian Day \\
\hline burst & Burst number & counts \\
\hline lat & Latitude & degree_north \\
\hline lon & Longitude & degree_east \\
\hline wh_4061 & Significant wave height & $\mathrm{m}$ \\
\hline wp_4060 & Mean wave period & $\mathrm{s}$ \\
\hline hght_18 & $\begin{array}{l}\text { Height of the sea surface relative to } \\
\text { sensor }\end{array}$ & $\mathrm{m}$ \\
\hline frequency & $\begin{array}{l}\text { Frequency at the center of each } \\
\text { frequency band }\end{array}$ & $\mathrm{Hz}$ \\
\hline direction & $\begin{array}{l}\text { Direction at the center of each direction } \\
\text { slice }\end{array}$ & degrees True \\
\hline mwh_4064 & Maximum wave height & $\mathrm{m}$ \\
\hline wp_peak & Peak wave period & $\mathrm{s}$ \\
\hline wvdir & $\begin{array}{l}\text { Peak wave direction from which waves } \\
\text { are propagating }\end{array}$ & degrees True \\
\hline dspec & Directional wave energy spectrum & $\mathrm{mm}^{2} / \mathrm{Hz} /$ degree \\
\hline pspec & $\begin{array}{l}\text { Pressure-derived non-directional wave } \\
\text { height spectrum }\end{array}$ & $\mathrm{mm} / \mathrm{sqrt}(\mathrm{Hz})$ \\
\hline sspec & $\begin{array}{l}\text { Surface-derived non-directional wave } \\
\text { height spectrum }\end{array}$ & $\mathrm{mm} / \mathrm{sqrt}(\mathrm{Hz})$ \\
\hline vspec & $\begin{array}{l}\text { Velocity-derived non-directional wave } \\
\text { height spectrum }\end{array}$ & $\mathrm{mm} / \mathrm{sqrt}(\mathrm{Hz})$ \\
\hline
\end{tabular}

\section{For the Sontek Argonaut:}

\begin{tabular}{|l|l|l|}
\hline Variable & Description & Units \\
\hline time & Time in Julian Days & Julian Day \\
\hline time2 & Time in Julian Days & msec of each Julian Day \\
\hline burst & Burst number & counts \\
\hline lat & Latitude & degree_north \\
\hline lon & Longitude & degree_east \\
\hline wh_4061 & Significant wave height & $\mathrm{m}$ \\
\hline hght_18 & $\begin{array}{l}\text { Height of the sea surface relative to } \\
\text { sensor }\end{array}$ & $\mathrm{m}$ \\
\hline hght_std & $\begin{array}{l}\text { Standard deviation of height of the sea } \\
\text { surface }\end{array}$ & $\mathrm{m}$ \\
\hline frequency & $\begin{array}{l}\text { Frequency at the center of each } \\
\text { frequency band }\end{array}$ & Hz \\
\hline
\end{tabular}




\begin{tabular}{|l|l|l|}
\hline wp_peak & Peak wave period & $\mathrm{s}$ \\
\hline pspec & $\begin{array}{l}\text { Pressure-derived non-directional wave } \\
\text { height spectrum }\end{array}$ & $\mathrm{mm} / \mathrm{sqrt}(\mathrm{Hz})$ \\
\hline
\end{tabular}

\section{For the Nortek AP:}

\begin{tabular}{|l|l|l|}
\hline Variable & Description & Units \\
\hline time & Time in Julian Days & Julian Day \\
\hline time2 & Time in Julian Days & msec of each Julian Day \\
\hline burst & Burst number & counts \\
\hline lat & Latitude & degree_north \\
\hline lon & Longitude & degree_east \\
\hline wh_4061 & Significant wave height & $\mathrm{m}$ \\
\hline hght_18 & $\begin{array}{l}\text { Height of the sea surface relative to } \\
\text { sensor }\end{array}$ & $\mathrm{m}$ \\
\hline frequency & $\begin{array}{l}\text { Frequency at the center of each } \\
\text { frequency band }\end{array}$ & $\mathrm{Hz}$ \\
\hline dfreq & Frequency band width & $\mathrm{Hz}$ \\
\hline wp_peak & Peak wave period & $\mathrm{s}$ \\
\hline wvdir & $\begin{array}{l}\text { Peak wave direction from which waves } \\
\text { are propagating }\end{array}$ & $\mathrm{degrees} \mathrm{True}$ \\
\hline spread & Peak spreading & degrees \\
\hline pspec & $\begin{array}{l}\text { Pressure-derived non-directional wave } \\
\text { height spectrum }\end{array}$ & $\mathrm{mm} / \mathrm{sqrt}(\mathrm{Hz})$ \\
\hline vspec & $\begin{array}{l}\text { Velocity-derived non-directional wave } \\
\text { height spectrum }\end{array}$ & $\mathrm{mm} / \mathrm{sqrt}(\mathrm{Hz})$ \\
\hline
\end{tabular}




\section{Appendix IV: M-files for RD Instruments ADCP}

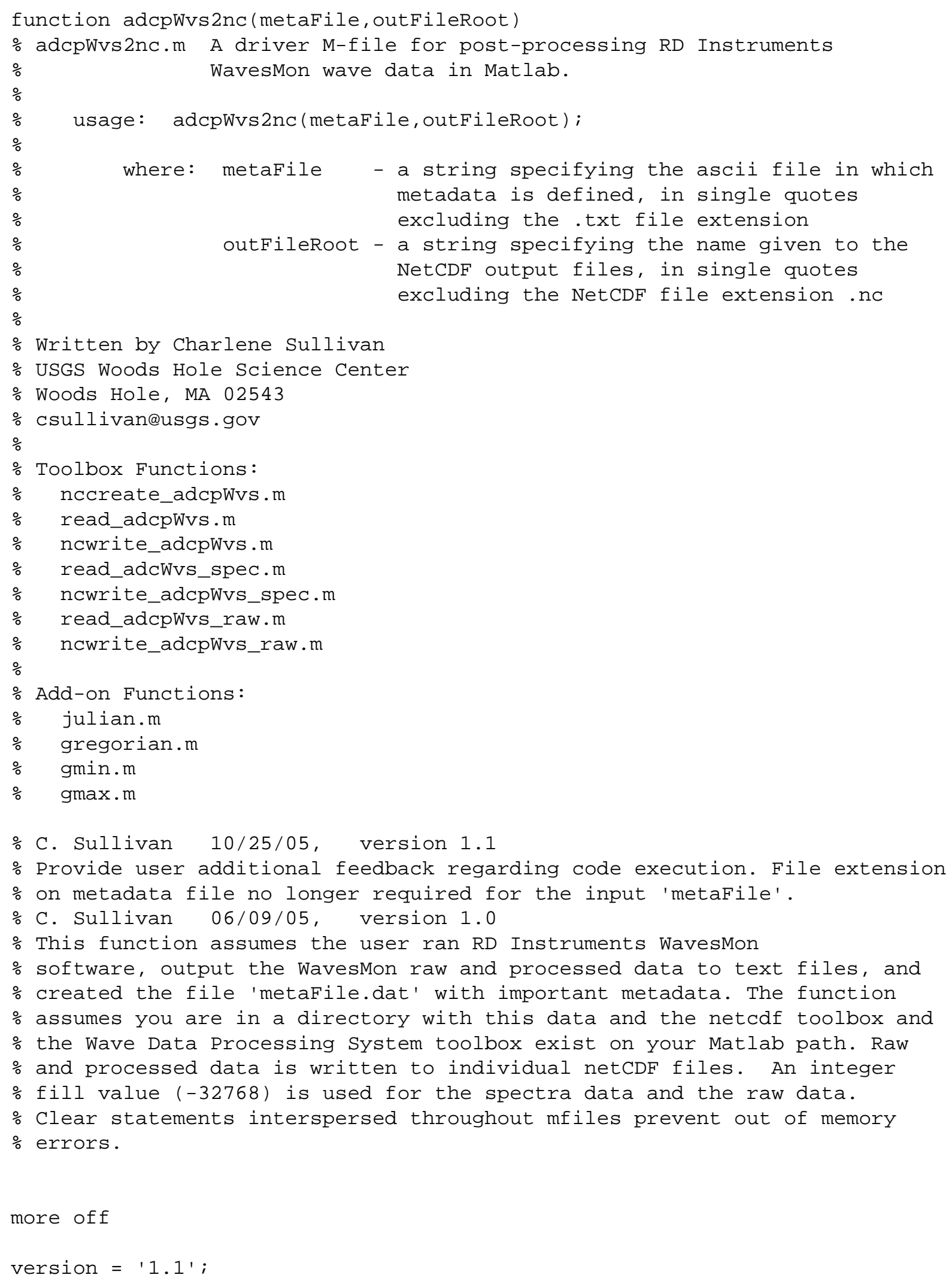




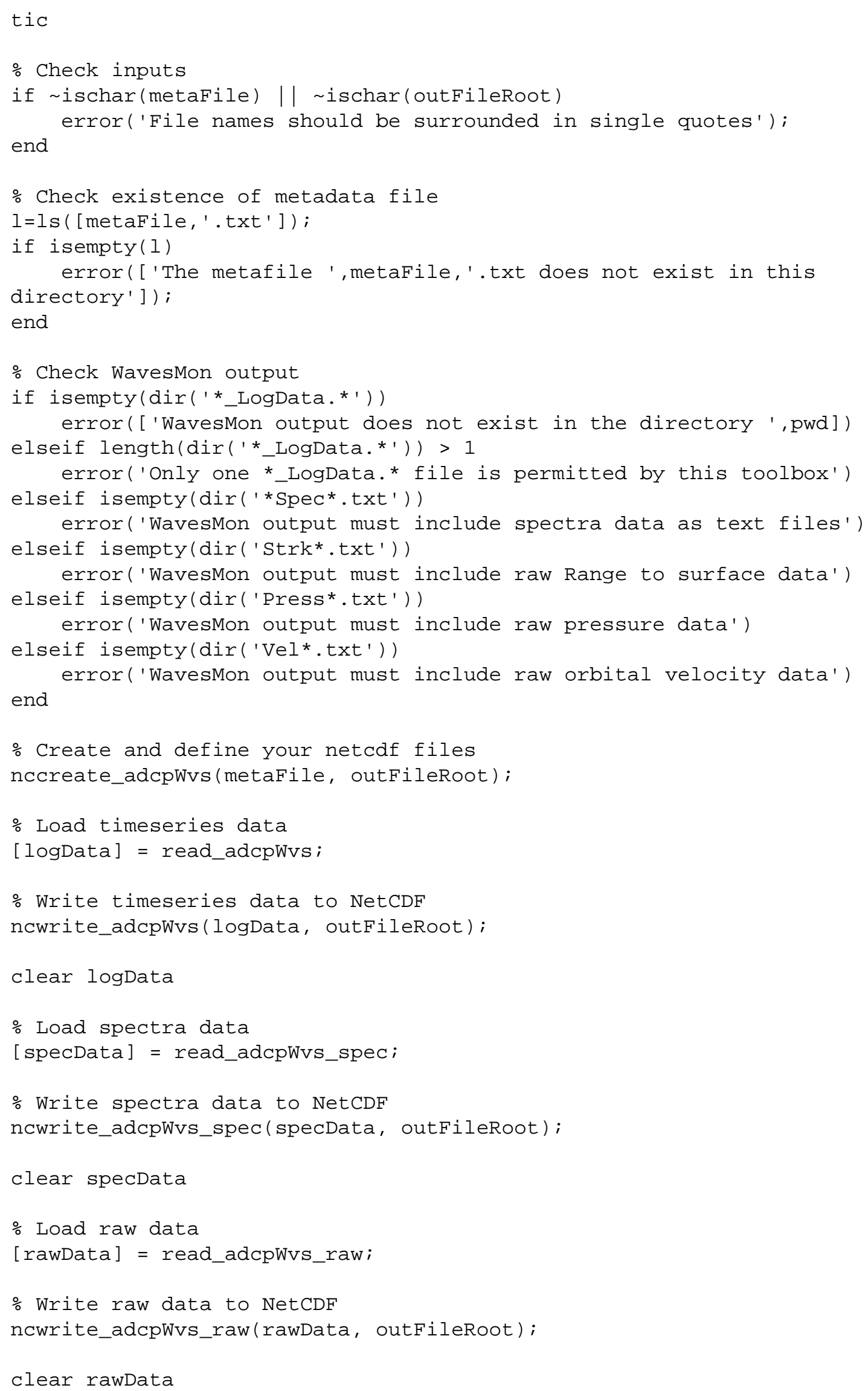


function nccreate_adcpWvs(metaFile, outFileRoot)

$\%$ nccreate_adcpWvs.m A function to create empty netCDF files that will $\%$ store RD Instruments ADCP wave data.

$\%$

usage: nccreate_adcpWvs(metaFile, outFileRoot);

where: metaFile - a string specifying the ascii file in which metadata is defined, in single quotes excluding the .txt file extension

outFileRoot - a string specifying the name given to the NetCDF output files, in single quotes excluding the NetCDF file extension .nc

Written by Charlene Sullivan

$\%$ USGS Woods Hole Science Center

$\%$ Woods Hole, MA 02543

\% csullivan@usgs.gov

$\%$ C. Sullivan $03 / 28 / 06$, version 1.2

\% Add EPIC keys for the variables: maximum wave height (new EPIC key 4064), $\%$ peak wave period (use existing EPIC key 4063), and peak wave direction $\%$ (use existing EPIC key 4062). Don't use EPIC keys for spectra variables, $\%$ because EPIC isn't suited for the spectral domain. Perhaps CF conventions $\%$ are better suited?

$\%$ C. Sullivan 10/25/05, version 1.1

$\%$ Provide user additional feedback regarding code execution. File extension on

$\%$ metadata file no longer required in the input metaFile. Changed DATA_TYPE $\%$ attribute description to be consistent with the documentation. Changed \% EPIC code and units on the variable lon to 502 and degree_east for $\%$ consistency $w /$ longitude as specified in the metafile where west is $\%$ negative. Add ADCP bin size attributes. Add selected bins for dspec and $\%$ vspec to attributes. Define direction variable for directional spectra. $\%$ Re-define frequency variable.

$\%$ C. Sullivan $06 / 09 / 05$, version 1.0 $\%$ Now defining both a raw data and processed data NetCDF files. Including $\%$ all information from the waves configuration file as metadata. Dimensions $\%$ depth $(=1)$, lat $(=1)$, and lon $(=1)$, beam $(=4)$, and beambin $(=12)$ are $\%$ hardwired.

version $=' 1.2$ ';

ncr $=$ netcdf $([$ outFileRoot, ' $r$-cal.nc'], 'clobber' $)$;

ncp $=\operatorname{netcdf}([$ outFileRoot, 'p-cal.nc'], 'clobber' $)$;

$\%$ Gather and write NetCDF metadata

[userMeta, userMetaDefs] = textread([metaFile, '.txt'],'\%s

$\% 63 c$ ', 'commentstyle', 'shell');

userMetaDefs = cellstr(userMetaDefs);

[wvmnMeta, wvmnMetaDefs] = read_WavesMon_config;

write_adcpWvs_meta(ncr, userMeta, userMetaDefs, wvmnMeta, wvmnMetaDefs) ncr.DATA_TYPE $=$ ncchar ('ADCP pressure and velocity timeseries');

ncr.VAR_DESC $=\operatorname{ncchar}($ 'press:vel:strk'); 
write_adcpWvs_meta(ncp, userMeta, userMetaDefs, wvmnMeta, wvmnMetaDefs) ncp.DATA_TYPE $=$ ncchar ('ADCP processed wave parameters and spectra'); ncp.VAR_DESC = ncchar ('Hs:Tp:Dp:Hmax:Tm:dspec:pspec: sspec: vspec ');

\% Define NetCDF dimensions

disp(['Defining NetCDF dimensions in ', outFileRoot, ' $r$-cal.nc and ', ... outFileRoot, 'p-cal.nc'])

define_adcpWvs_dims(ncr);

define_adcpWvs_dims(ncp);

$\%$ Define NetCDF variables

disp(['Defining NetCDF variables in ', outFileRoot, 'r-cal.nc and ', ...

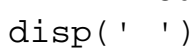
outFileRoot, 'p-cal.nc'])

define_adcpWvs_vars(ncr);

define_adcpWvs_vars(ncp);

endef(ncr);

ncr $=$ close $($ ncr $)$;

endef(ncp);

ncp $=\operatorname{close}($ ncp $)$;

return

\% -...-.- Subfunction: Gather WavesMon configuration data -........ \% function [wvmnMeta, wvmnMetaDefs]=read_WavesMon_config;

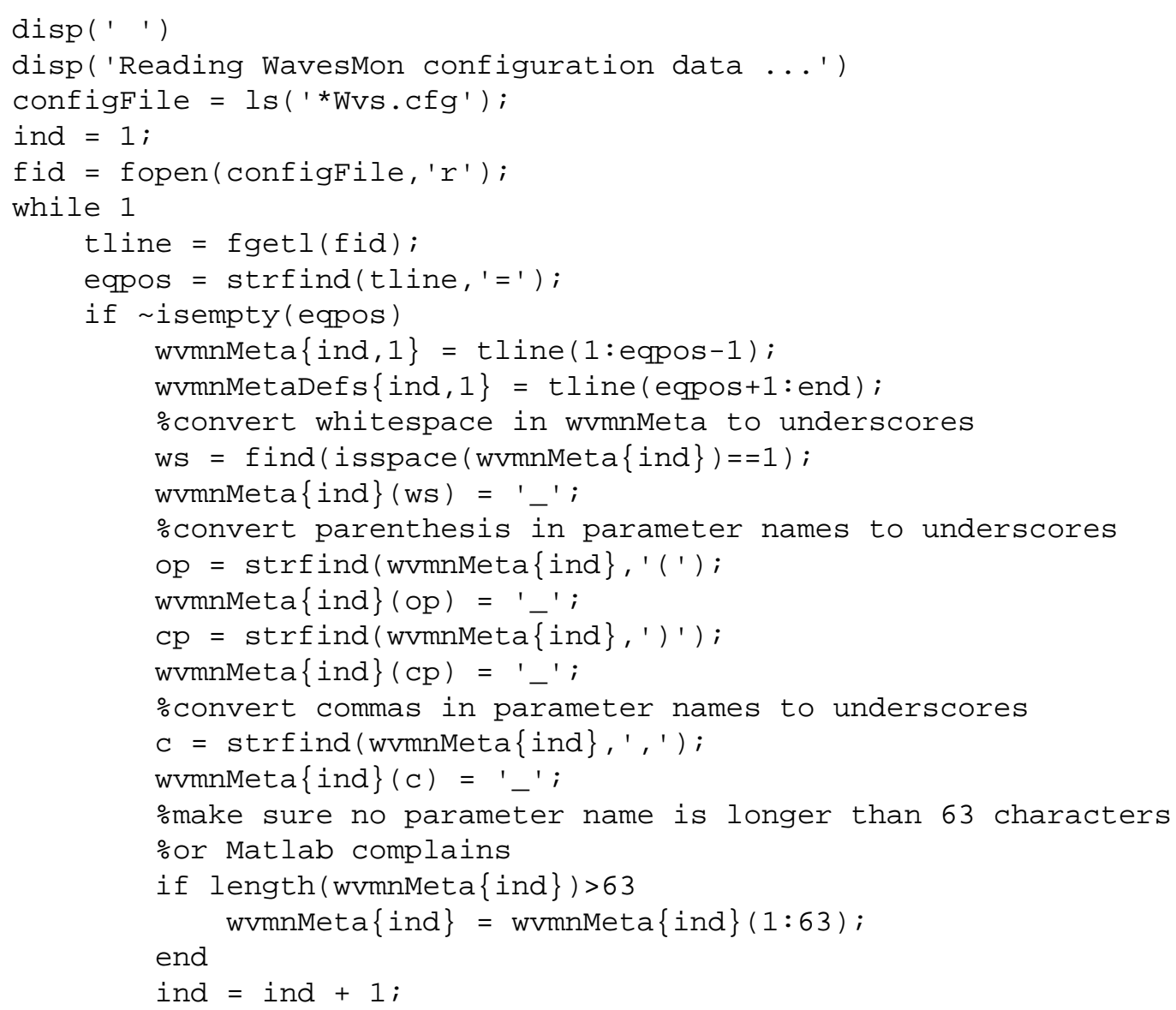




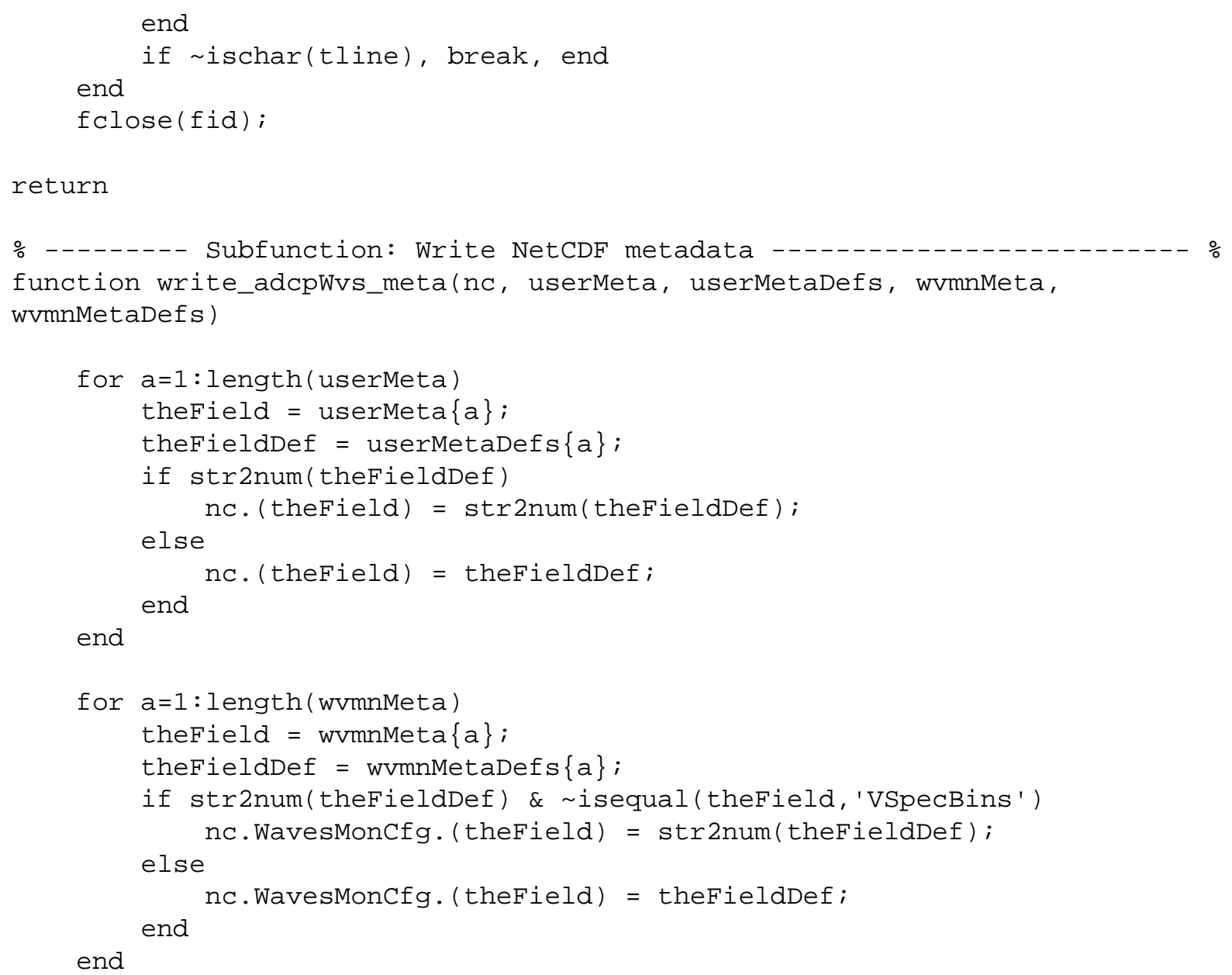




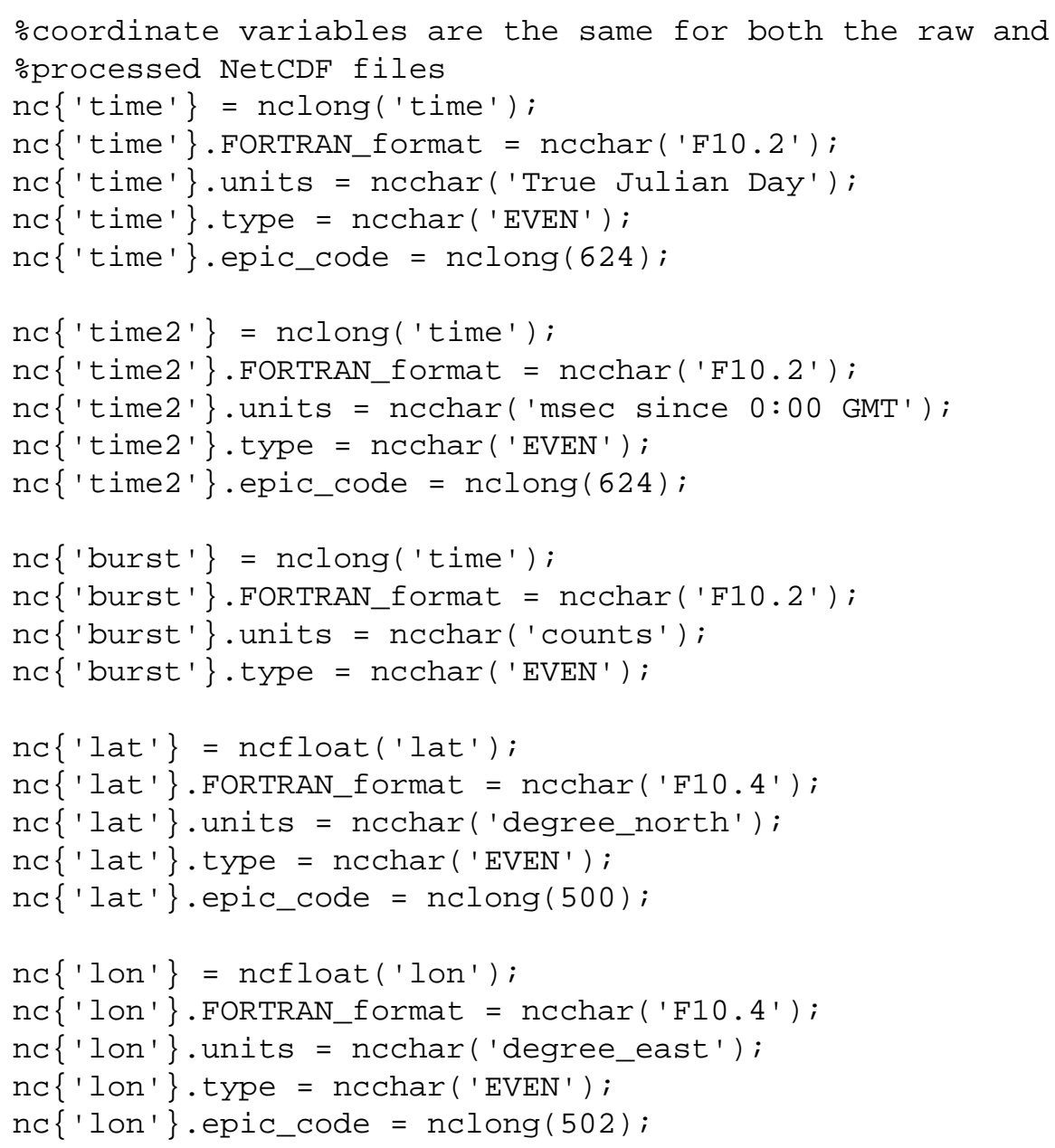

if strcmp(nc.DATA_TYPE(:), 'ADCP processed wave parameters and spectra') \%Record variables for ONLY the processed data NetCDF file. \%EPIC variables nc $\{$ 'wh_4061' $\}=$ ncdouble ('time', 'lat', 'lon'); nc $\{$ 'wh_4061'\}.long_name=ncchar ('Significant Wave Height $(m)$ '); 


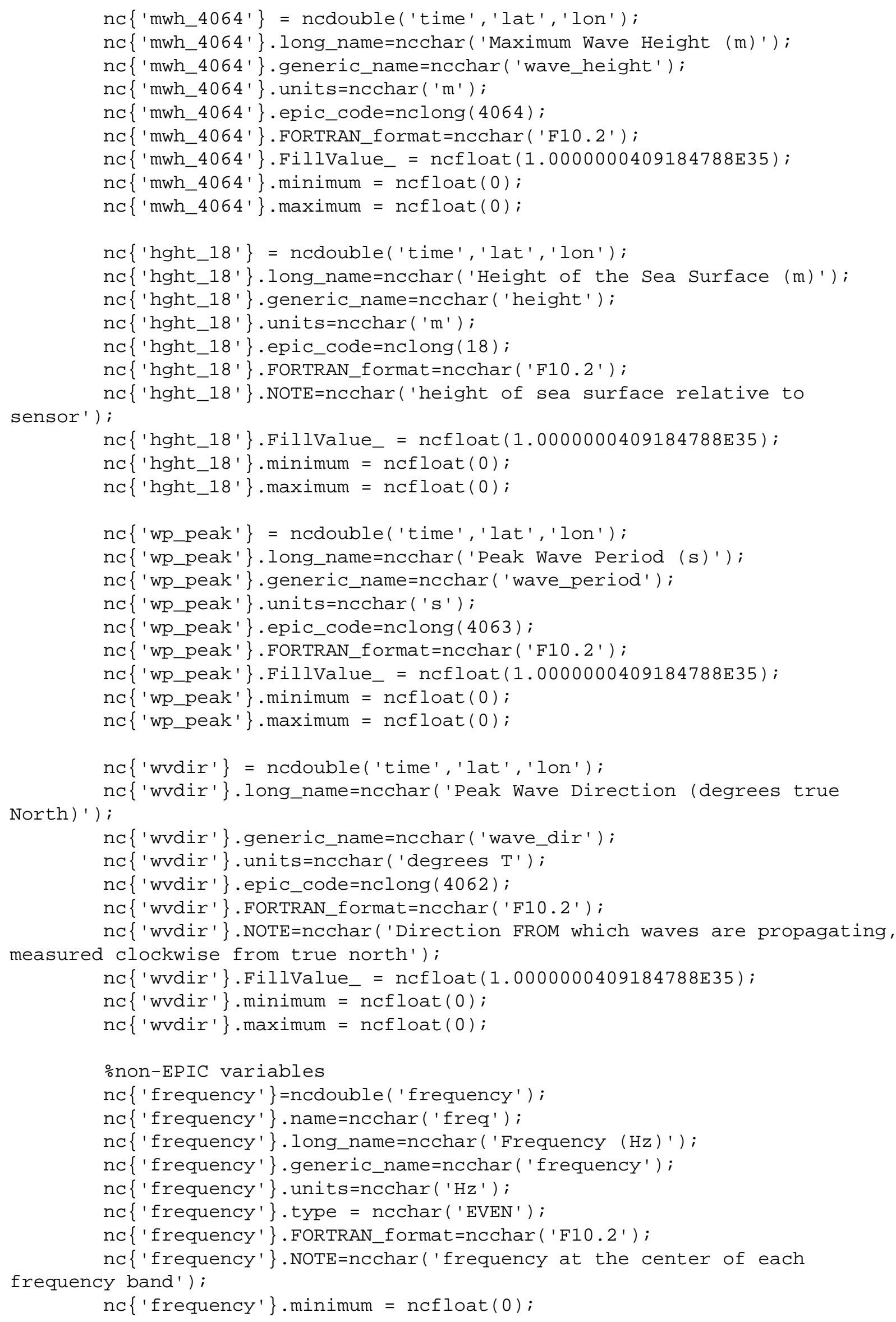


$\mathrm{nc}\{$ 'frequency' $\}$. maximum $=\operatorname{ncfloat}(\Theta)$;

nc $\{$ 'direction' $\}=$ ncdouble( 'direction' ');

nc $\{$ 'direction' $\}$. name=ncchar ('dir');

nc $\{$ 'direction'\}. long_name=ncchar ('Direction (degrees T)');

nc $\{$ 'direction' $\}$.generic_name=ncchar ('direction');

nc $\{$ 'direction' $\}$. units=ncchar ('degrees $\left.T^{\prime}\right)$;

nc $\{$ 'direction' $\}$.type = ncchar ('EVEN');

nc $\{$ 'direction' $\}$.FORTRAN_format=ncchar ('F10.2');

slice' );

nc $\{$ 'direction'\}. NOTE=ncchar ('direction at center of each direction

nc $\{$ 'direction' $\}$. minimum $=\operatorname{ncfloat}(\theta)$;

nc $\{$ 'direction' $\}$. maximum $=\operatorname{ncfloat}(\odot)$;

nc $\{$ 'dspec ' $\}=$ ncshort ('time' , 'frequency' , 'direction', 'lat ', 'lon ' );

$n c\{$ 'dspec' $\}$. name=ncchar ( 'dspec');

nc $\{$ 'dspec'\}.long_name=ncchar ('Directional wave Energy spectrum

$\left.(\mathrm{mm} \wedge 2 / \mathrm{Hz} / \text { degree })^{\prime}\right)$;

nc $\{$ 'dspec'\}.generic_name=ncchar ('directional spectrum');

$n c\{$ 'dspec' $\}$. units=ncchar $\left(\right.$ ' $m m^{\wedge} 2 / \mathrm{Hz} /$ degree' $)$;

nc $\{$ 'dspec' $\}$. DspecBins=nc. WavesMonCfg.DirSpecBins ( : );

nc $\{$ 'dspec'\}. bin_size=nc. ADCPBinSize( : );

nc $\{$ 'dspec' $\}$.FORTRAN_format=ncchar ( 'F10.2' );

nc $\{$ 'dspec' $\}$.Fillvalue $=$ ncshort $(-32768)$;

$\mathrm{nc}\{$ 'dspec' $\}$. minimum $=$ ncfloat $(\odot)$;

$n c\{$ 'dspec' $\} \cdot \operatorname{maximum}=\operatorname{ncfloat}(\Theta)$;

nc $\{$ 'pspec' $\}=$ ncshort ('time', 'frequency', 'lat ', 'lon');

nc $\{$ 'pspec' $\}$. name=ncchar ( 'pspec' );

nc $\{$ 'pspec'\}. long_name=ncchar ('Pressure-derived Non-directional Wave

Height Spectrum (mm/sqrt(Hz))');

nc $\{$ 'pspec'\}.generic_name=ncchar ('pressure spectrum');

nc $\{$ 'pspec' $\}$. units=ncchar ( 'mm/sqrt (Hz)' );

nc $\{$ 'pspec' $\}$.FORTRAN_format=ncchar ( 'F10.2' );

nc $\{$ 'pspec' $\}$. FillValue ${ }_{-}=\operatorname{ncshort}(-32768)$;

nc $\{$ 'pspec' $\}$. minimum $=\operatorname{ncfloat}(\Theta)$;

nc $\{$ 'pspec' $\}$. maximum $=\operatorname{ncfloat}(\odot)$;

nc $\{$ 'sspec' $\}=$ ncshort ('time', 'frequency', 'lat ', 'lon' );

nc $\{$ 'sspec' $\}$. name=ncchar ( 'sspec') ;

nc $\{$ 'sspec' $\}$.long_name=ncchar ( 'Surface-derived Non-directional Wave

Height Spectrum (mm/sqrt $\left.(\mathrm{Hz}))^{\prime}\right)$;

nc $\{$ 'sspec'\}.generic_name=ncchar ('surface spectrum');

nc $\{$ 'sspec' $\}$. units=ncchar ('mm/sqrt (Hz)' );

nc $\{$ 'sspec' $\}$.FORTRAN_format=ncchar ('F10.2');

nc $\{$ 'sspec' $\}$. FillValue $=$ ncshort $(-32768)$;

$\mathrm{nc}\{$ 'sspec' $\} \cdot \operatorname{minimum}=\operatorname{ncfloat}(0)$;

nc $\{$ 'sspec' $\}$. maximum $=\operatorname{ncfloat}(\odot)$;

nc $\{$ 'vspec' $\}=$ ncshort ('time', 'frequency', 'lat', 'lon');

nc $\{$ 'vspec' $\}$. name=ncchar ( 'vspec');

nc $\{$ 'vspec'\}. long_name=ncchar ('Velocity-derived Non-directional wave

Height Spectrum (mm/sqrt(Hz))');

nc $\{$ 'vspec'\}.generic_name=ncchar ('velocity spectrum');

nc $\{$ 'vspec' $\}$. units=ncchar ( 'mm/sqrt (Hz)' );

nc $\{$ 'vspec' $\}$. VspecBins=nc. WavesMonCfg.VSpecBins ( : ); 
nc $\{$ 'vspec' $\}$.bin_size=nc.ADCPBinSize ( : );

nc $\{$ 'vspec' $\}$. FORTRAN_format=ncchar ( 'F10.2');

nc $\{$ 'vspec' $\}$. Fillvalue $=\operatorname{ncshort}(-32768)$;

$\mathrm{nc}\{$ 'vspec' $\}$. minimum $=\operatorname{ncfloat}(\Theta)$;

$\mathrm{nc}\{$ 'vspec' $\} \cdot \operatorname{maximum}=\operatorname{ncfloat}(\odot)$;

elseif strcmp(nc.DATA_TYPE(:), 'ADCP pressure and velocity timeseries') \%Record variables for ONLY the raw data NetCDF file. There are no \%EPIC-compatible variables for these quantities.

nc $\{$ 'sample' $\}=$ nclong ('sample');

nc $\{$ 'sample' $\}$. FORTRAN_format $=$ ncchar ('F10.2');

nc $\{$ 'sample' $\}$. units = ncchar ('counts');

nc $\{$ 'sample' $\}$.type = ncchar ( 'EVEN' $)$;

nc $\{$ 'press' $\}=$ ncshort ('time', 'sample', 'lat', 'lon');

nc $\{$ 'press' $\}$. name $=$ ncchar $($ 'press' $)$;

nc $\{$ 'press'\}. long_name=ncchar ('Pressure Sensor Derived Depth (mm)');

nc $\{$ 'press'\}.generic_name=ncchar ('pressure time series');

nc $\{$ 'press' $\}$. units=ncchar $($ 'mm' $)$;

nc $\{$ 'press' $\}$.FORTRAN_format=ncchar ('F10.2');

nc $\{$ 'press' $\}$.FillValue ${ }_{-}=\operatorname{ncshort}(-32768)$;

nc $\{$ 'press' $\}$. minimum $=$ ncfloat $(0)$;

nc $\{$ 'press' $\}$. maximum $=\operatorname{ncfloat}(\odot)$;

nc $\{$ 'strk' $\}=$ ncshort ('time', 'sample', 'beam', 'lat', 'lon');

nc $\{$ 'strk'\} . name=ncchar ( 'strk');

nc $\{$ 'strk'\} . long_name=ncchar ('Along-Beam Surface Track (mm)');

nc $\{$ 'strk'\}.generic_name=ncchar ('surface track time series');

nc $\{$ 'strk' $\}$. units=ncchar ( 'mm' );

nc $\{$ 'strk'\}.FORTRAN_format=ncchar ( 'F10.2' );

nc $\left\{\right.$ 'strk'\}.Fillvalue ${ }_{-}=\operatorname{ncshort}(-32768)$;

nc $\{$ 'strk' $\}$. minimum $=\operatorname{ncfloat}(\Theta)$;

nc $\{$ 'strk' $\}$. maximum $=\operatorname{ncfloat}(\Theta)$;

nc $\{$ 'vel' $\}=$ ncshort( 'time', 'sample', 'beambin', 'lat', 'lon');

nc $\{$ 'vel' $\}$. name=ncchar ('vspec');

nc $\{$ 'vel'\}. long_name=ncchar ('Along-Beam Velocity (mm/s)');

nc $\{$ 'vel'\}.generic_name=ncchar ('velocity time series');

nc $\{$ 'vel' $\}$. units=ncchar ( 'mm/s');

nc $\{$ 'vel' $\}$. bin_size=nc. ADCPBinSize ( : );

nc $\{$ 'vel' $\}$.FORTRAN_format=ncchar ( 'F10.2' );

nc $\{$ 'vel' $\}$.Fillvalue $=\operatorname{ncshort}(-32768)$;

nc $\{$ 'vel' $\}$. minimum $=\operatorname{ncfloat}(\Theta)$;

$n c\{$ 'vel' $\} \cdot \operatorname{maximum}=\operatorname{ncfloat}(\Theta)$;

end

return 


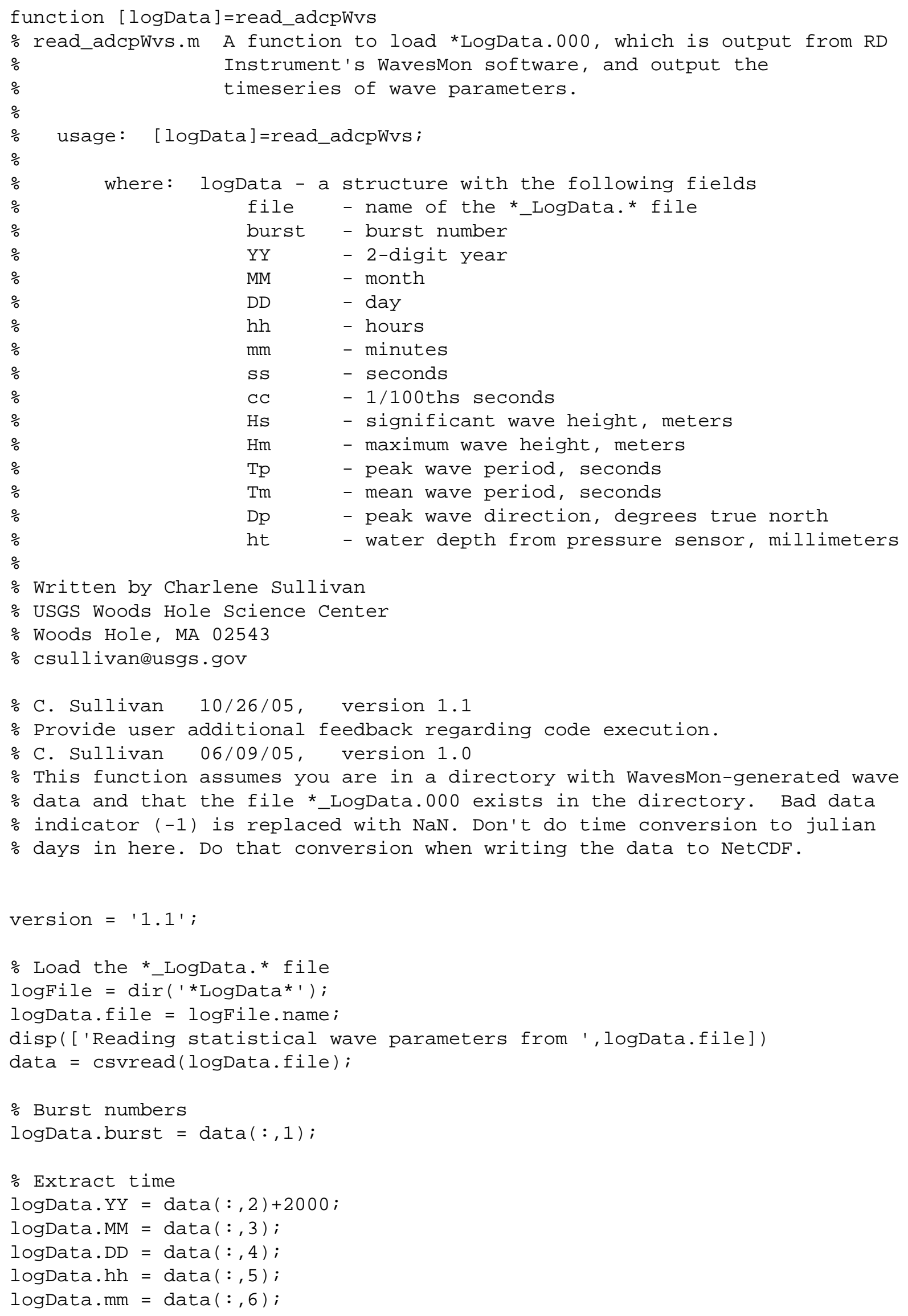




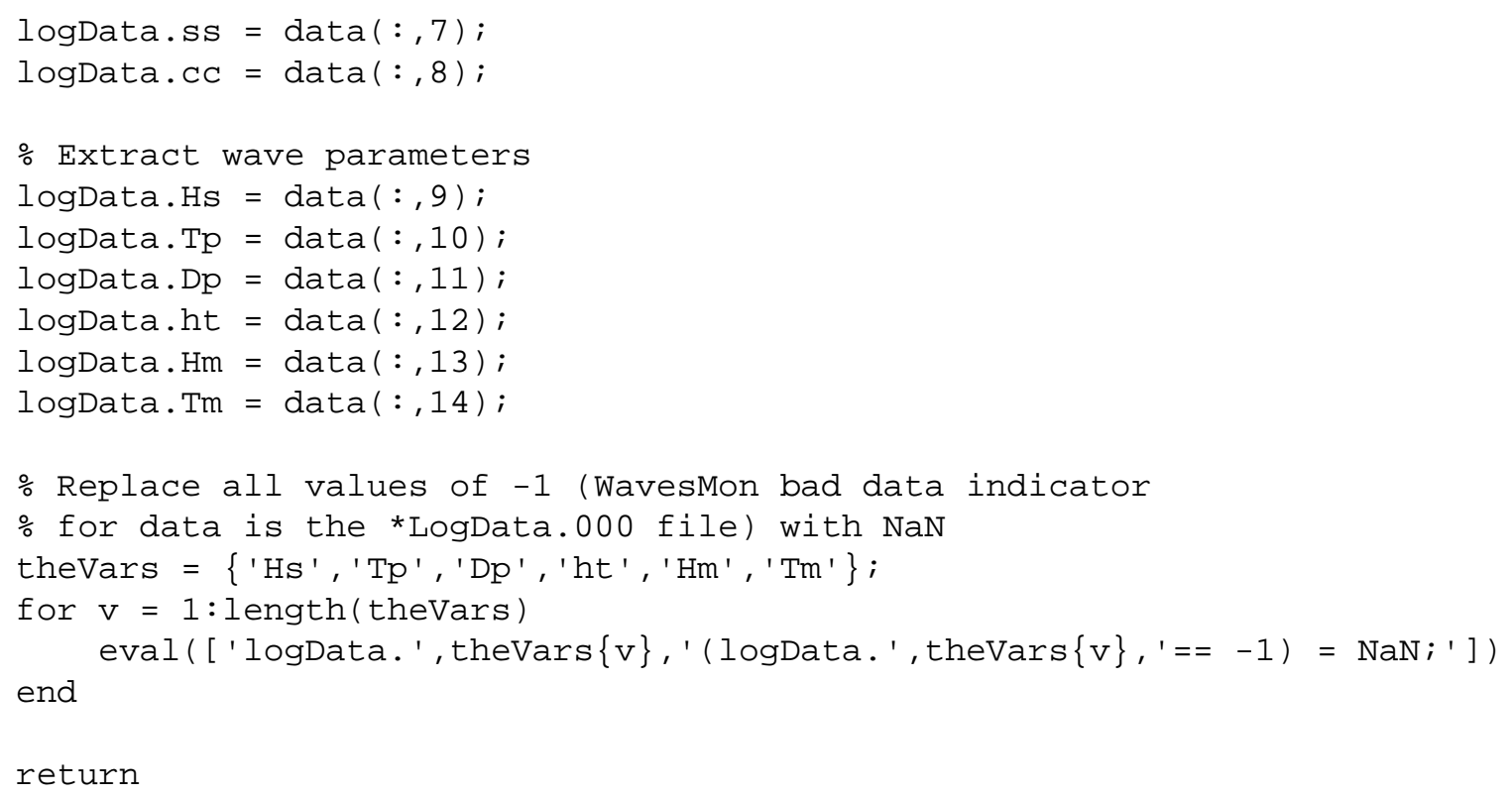




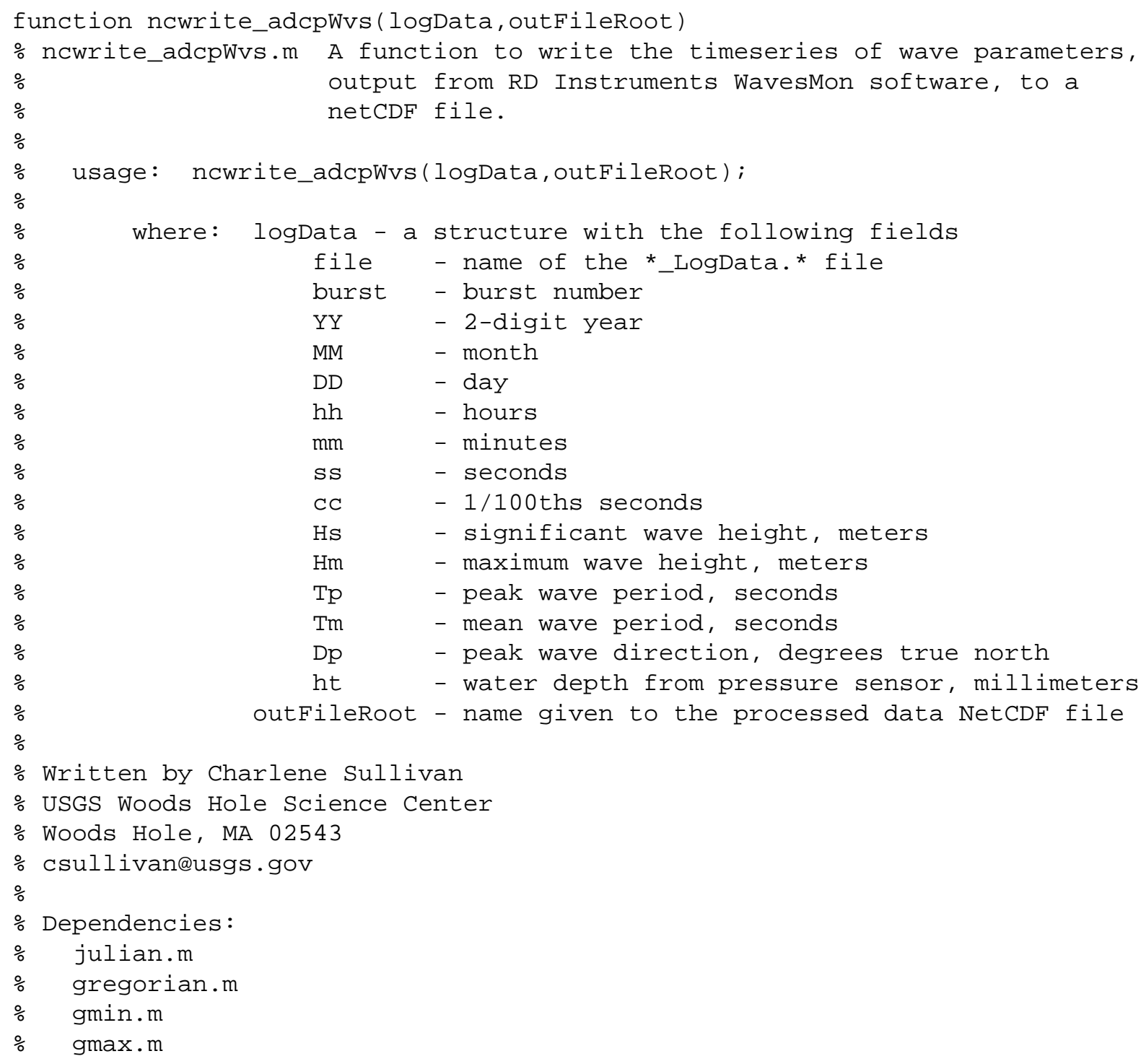

$\%$ C. Sullivan $03 / 29 / 06$, version 1.2

\% Now using EPIC variable mwh_4064 for maximum wave height. Reverse the

$\%$ chronology on the history attribute so the most recent processing step is $\%$ listed first.

$\%$ C. Sullivan 10/26/05, version 1.1

$\%$ Provide user additional feedback regarding code execution. Use NetCDF

$\%$ variable objects to handle statistical wave parameters.

$\%$ C. Sullivan $06 / 09 / 05$, version 1.0

$\%$ Do time conversion to julian days in here. Calculate min/max values and $\%$ replacing nan's with the NetCDF fill value.

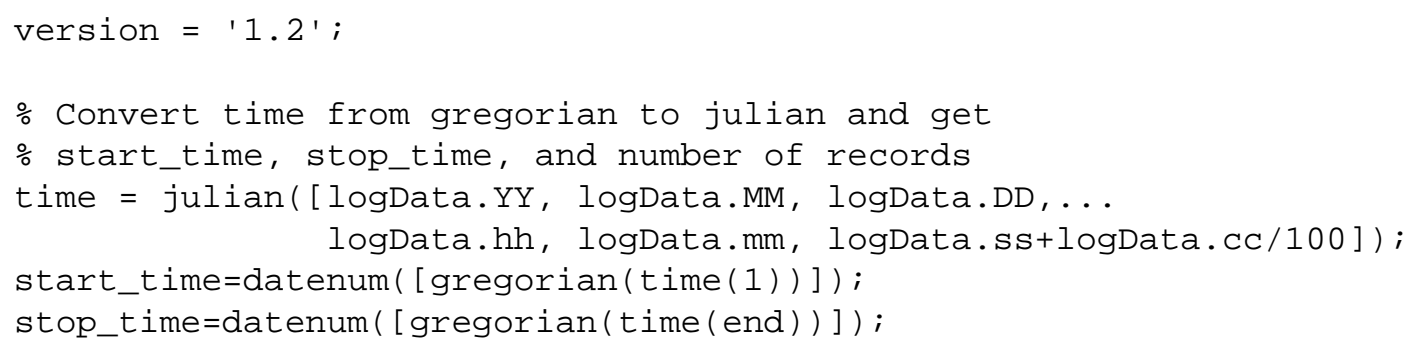




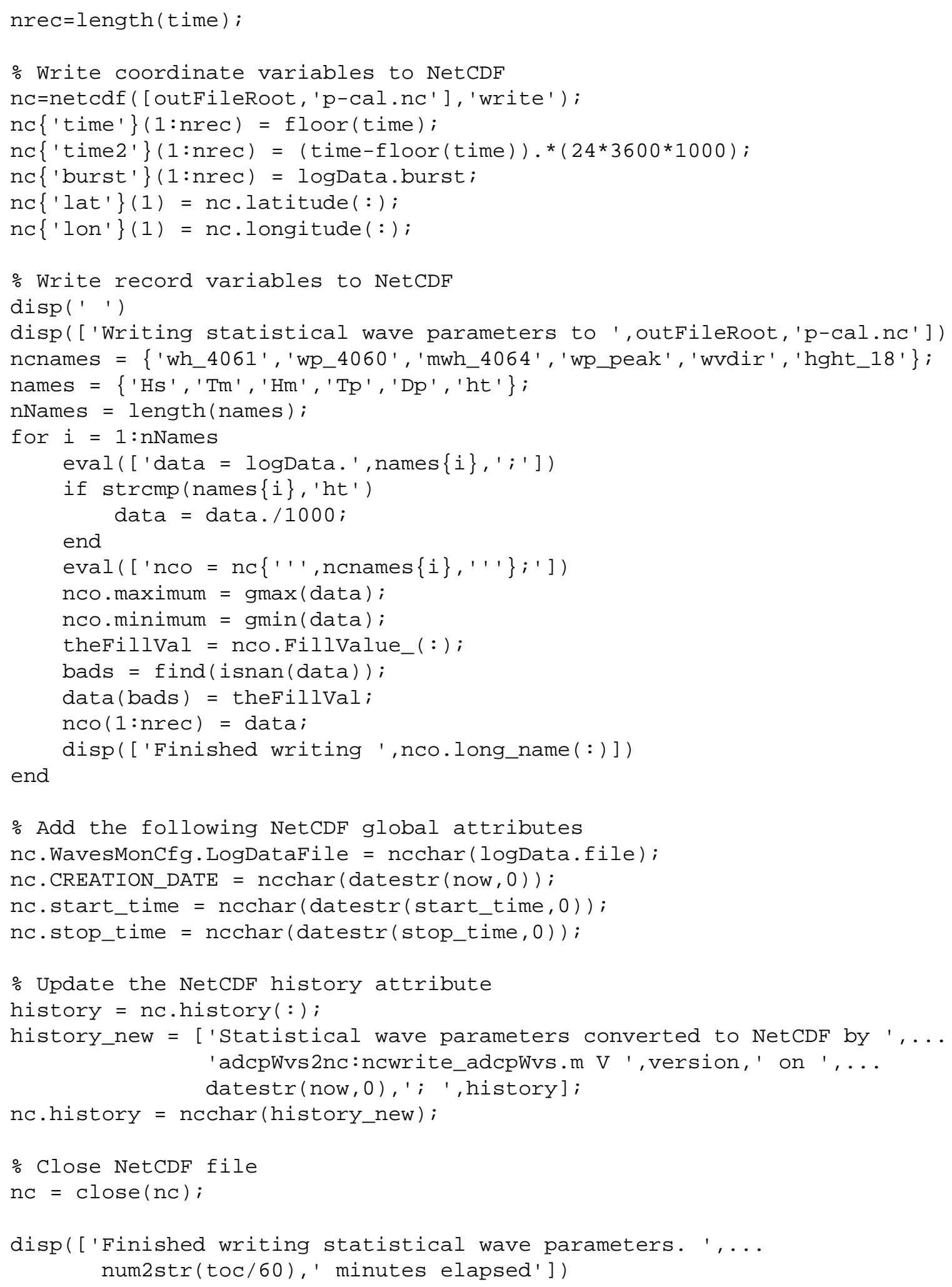

return 


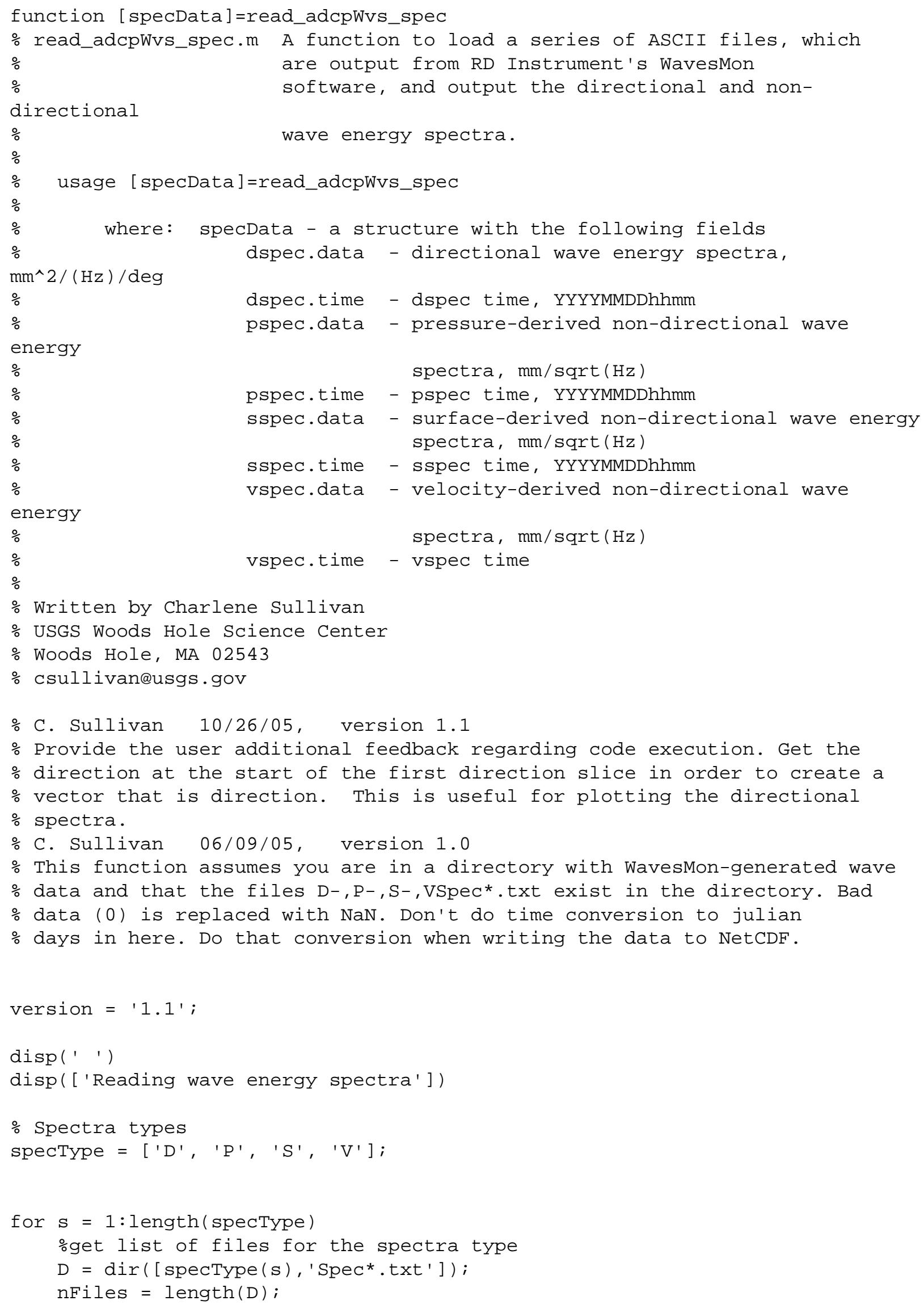




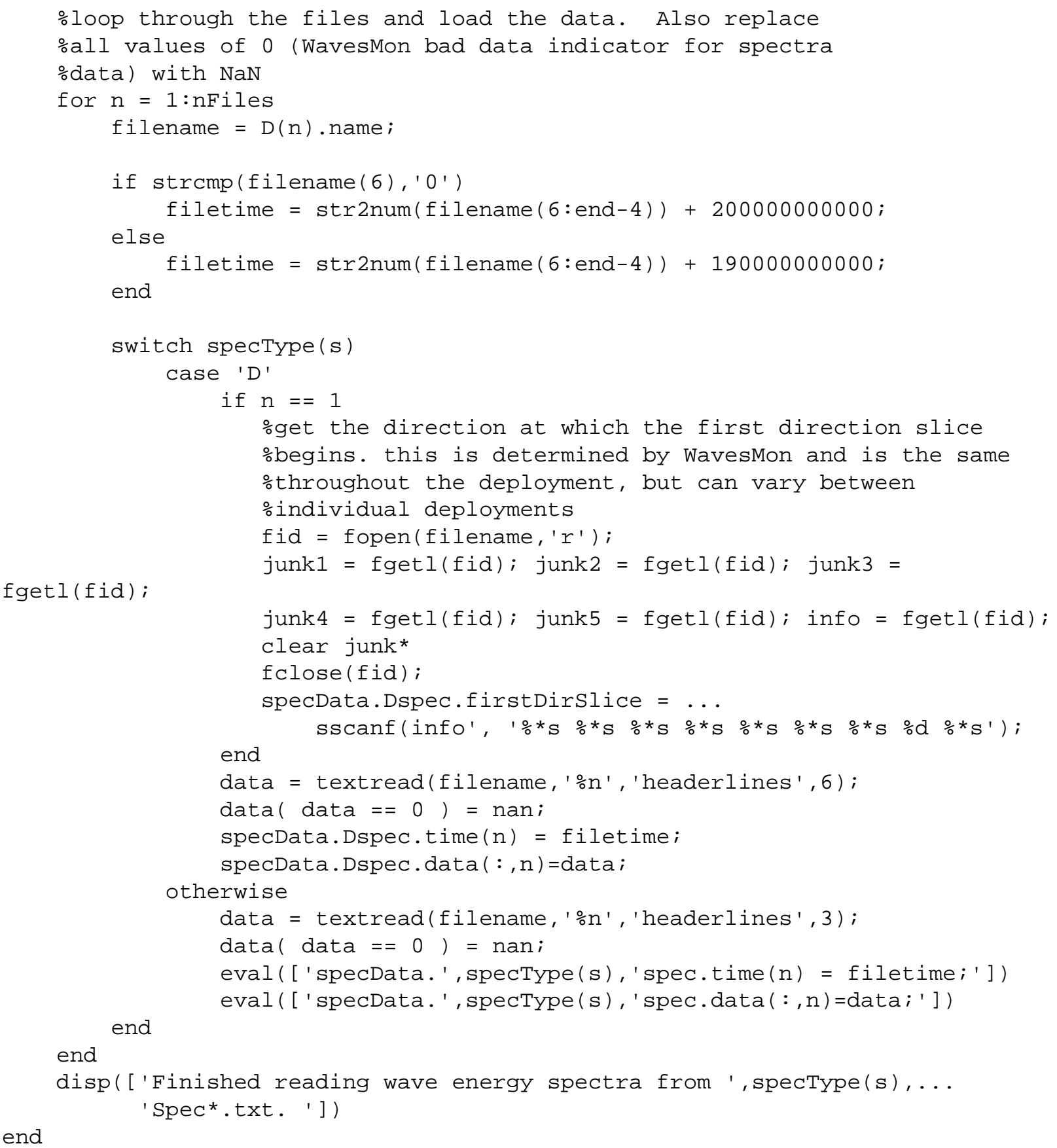

return 


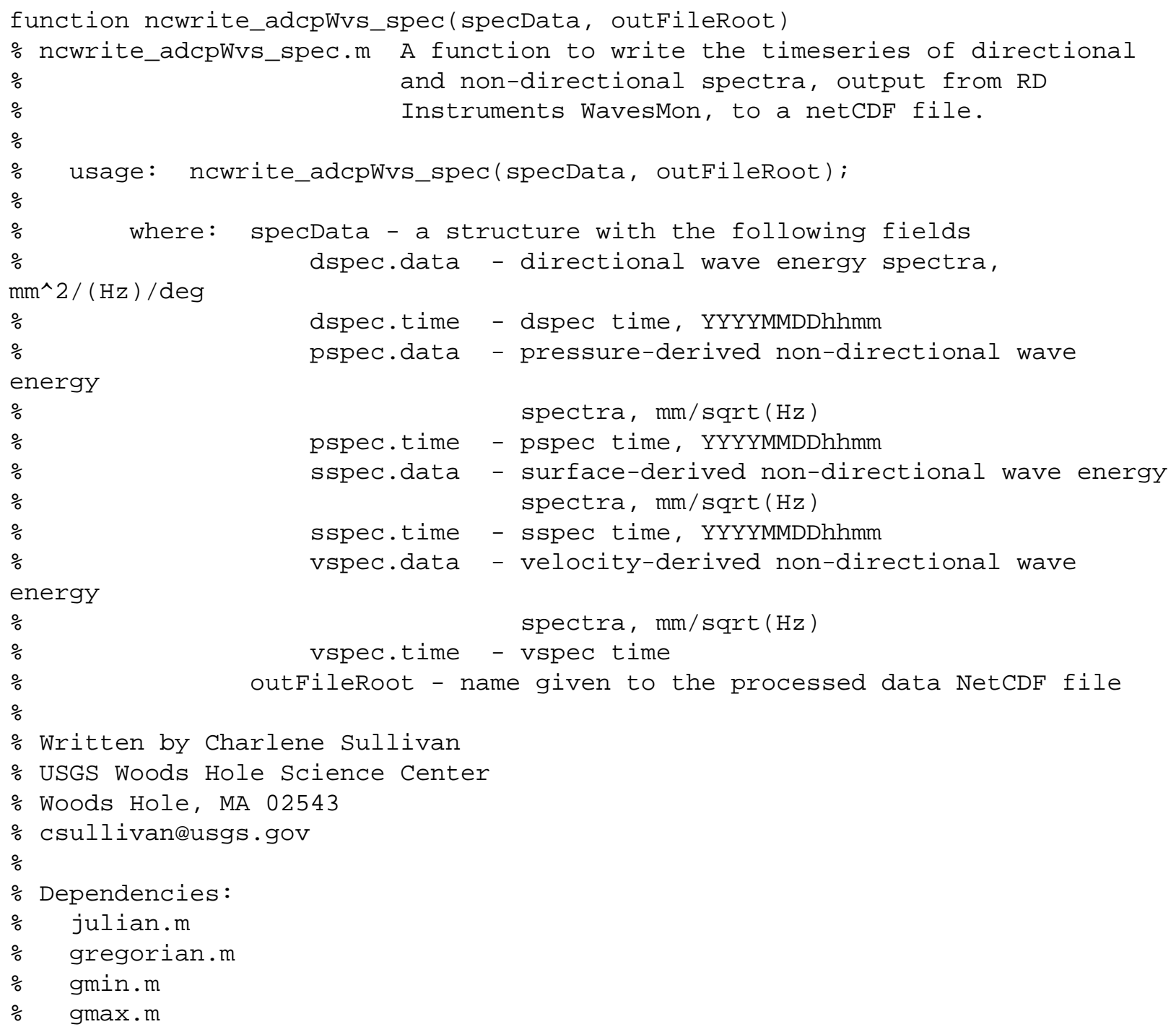

\% C. Sullivan 03/29/06, version 1.3

$\%$ Reverse the chronology on the history attribute so the most recent $\%$ processing step is listed first. In calculating max and min attributes $\%$ for the spectra, calculate the max and min over time for each frequency $\%$ bin.

\% C. Sullivan $03 / 08 / 06$, version 1.2

$\%$ The function no longer assumes the times of the spectra data include $\%$ seconds. When aligning spectra data with wave parameters just use year, $\%$ month, day, hour, and minute.

$\%$ C. Sullivan 10/26/05, version 1.1

$\%$ Provide the user more feedback regarding code execution. Define the $\%$ variable direction for the directional spectra. This direction is the $\%$ direction at the center of each directional slice for the directional $\%$ spectra.

\% C. Sullivan $06 / 09 / 05$, version 1.0

$\%$ This function assumes times on the spectra data include the 30 seconds $\%$ that the times in the *_LogData* files have.

version $=' 1.3 '$; 


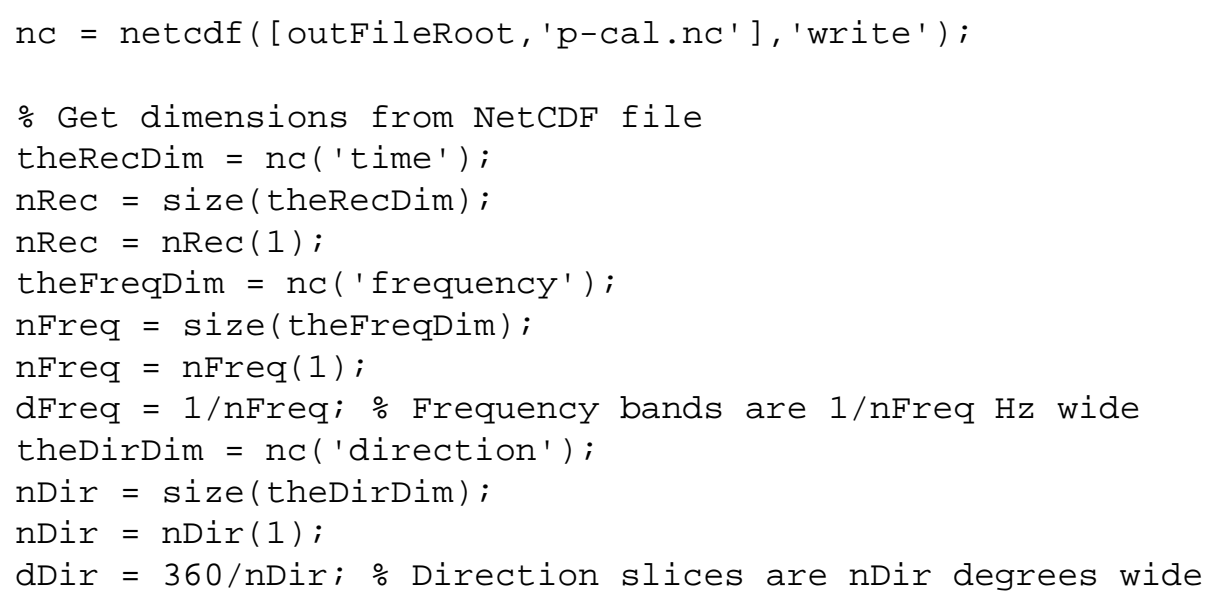


nFreq)' ;

dimension

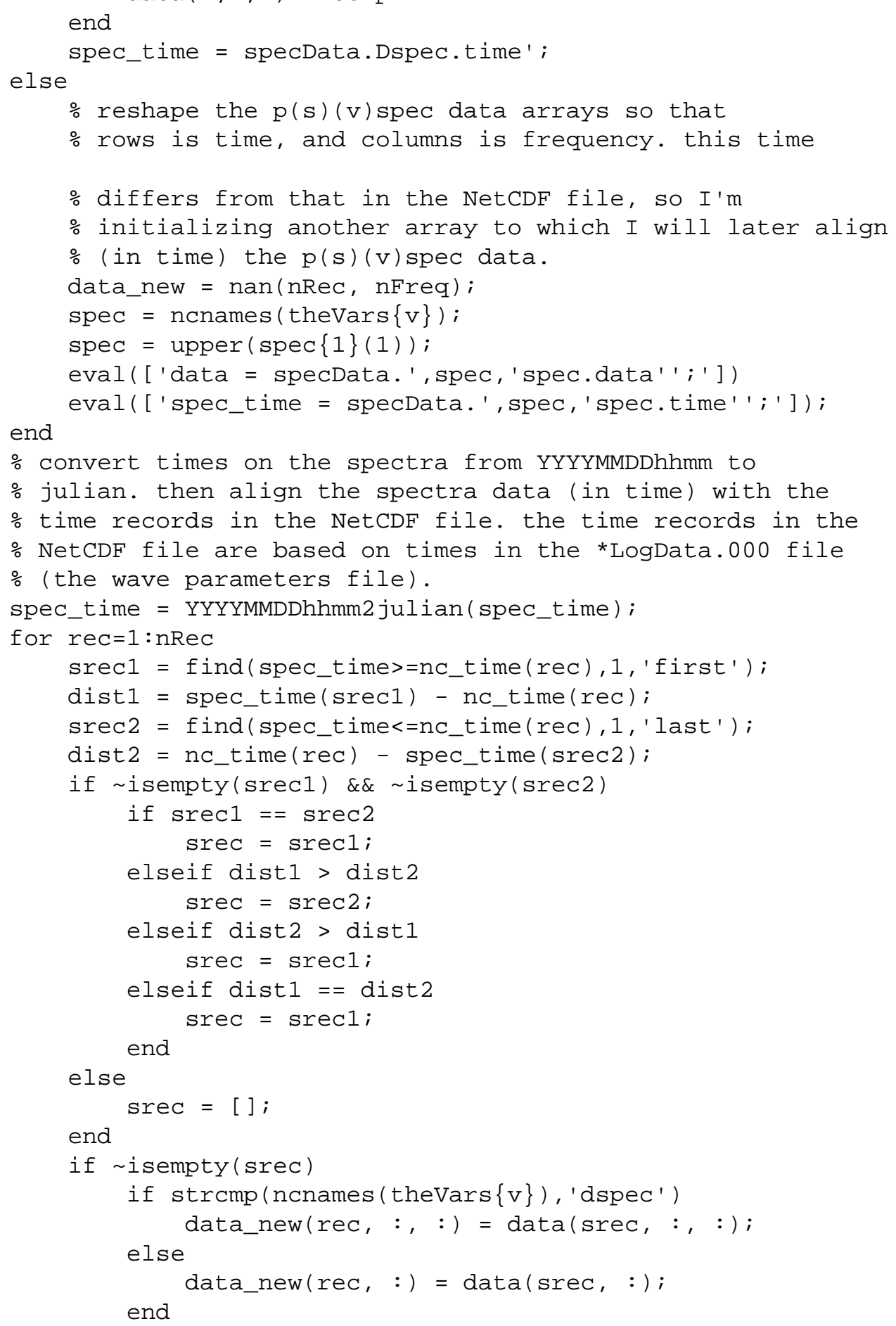




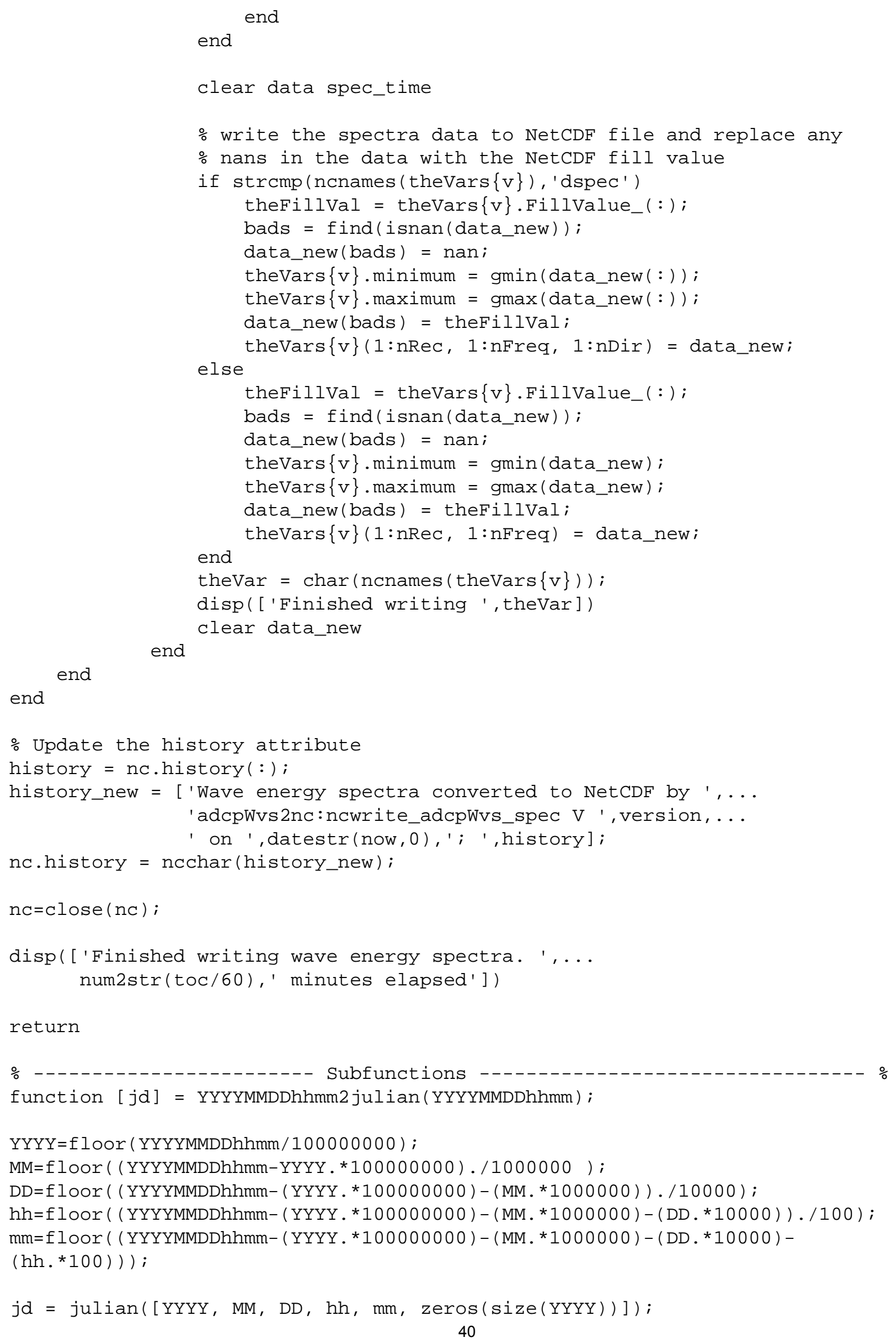


return 


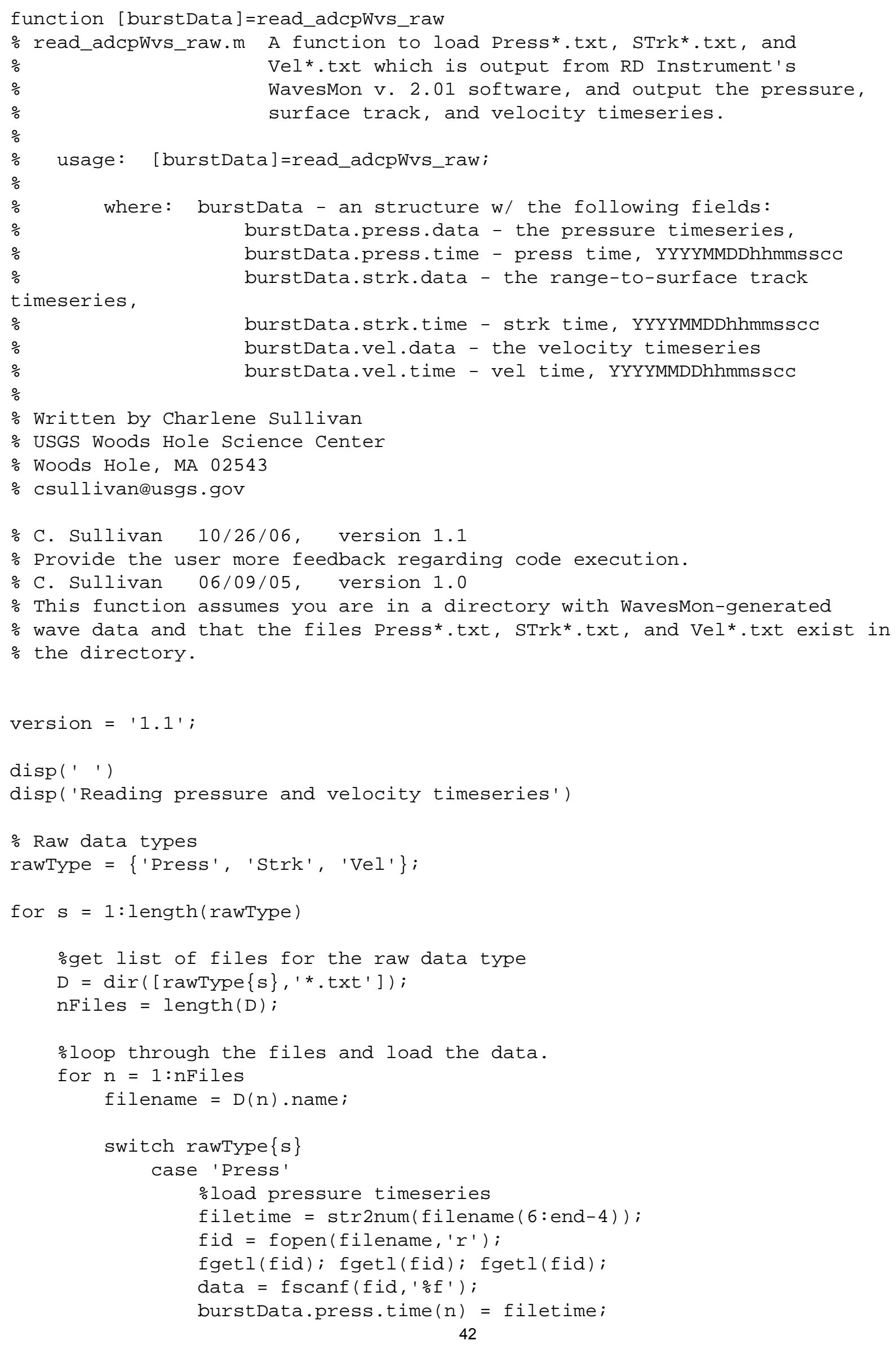




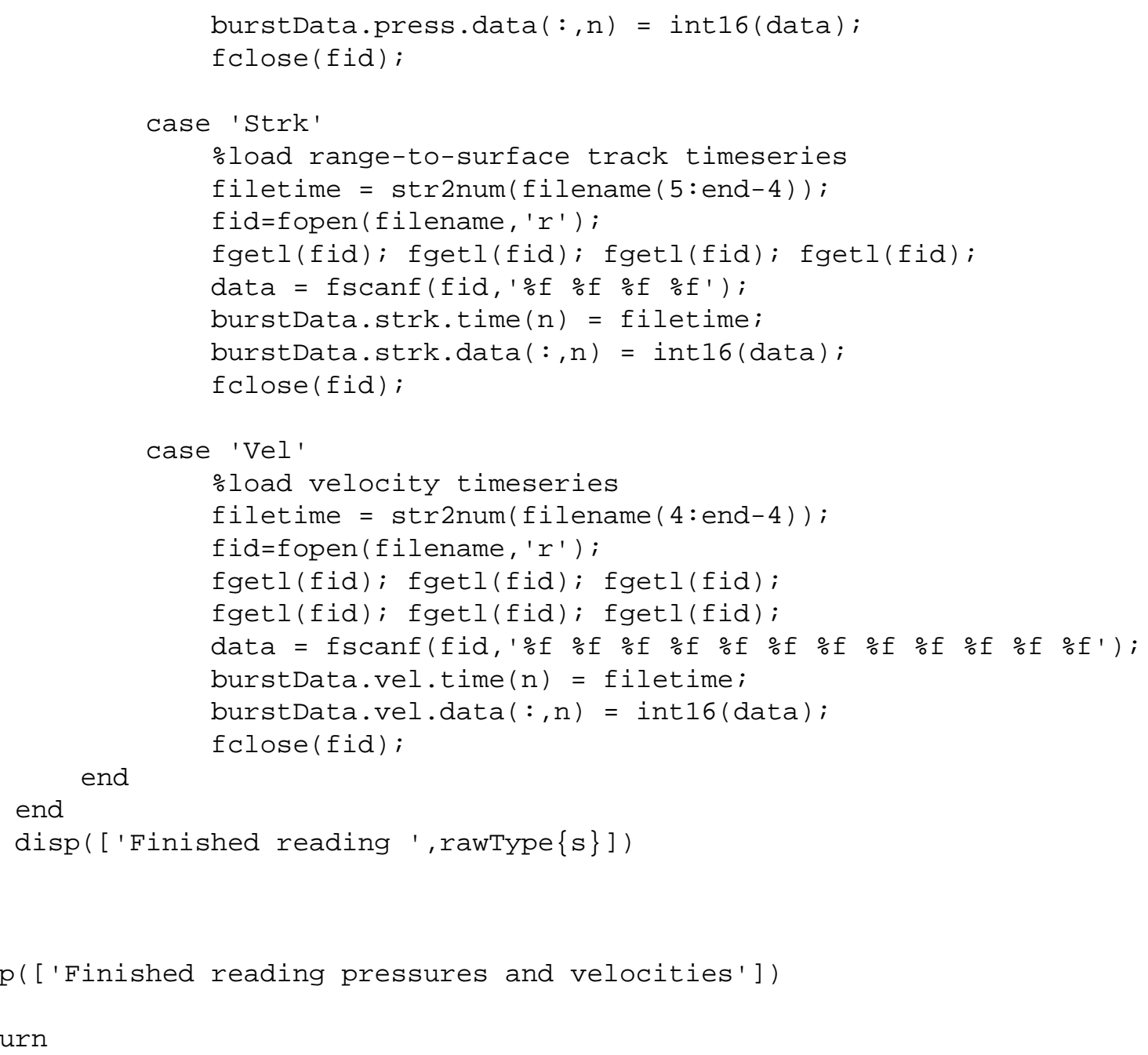




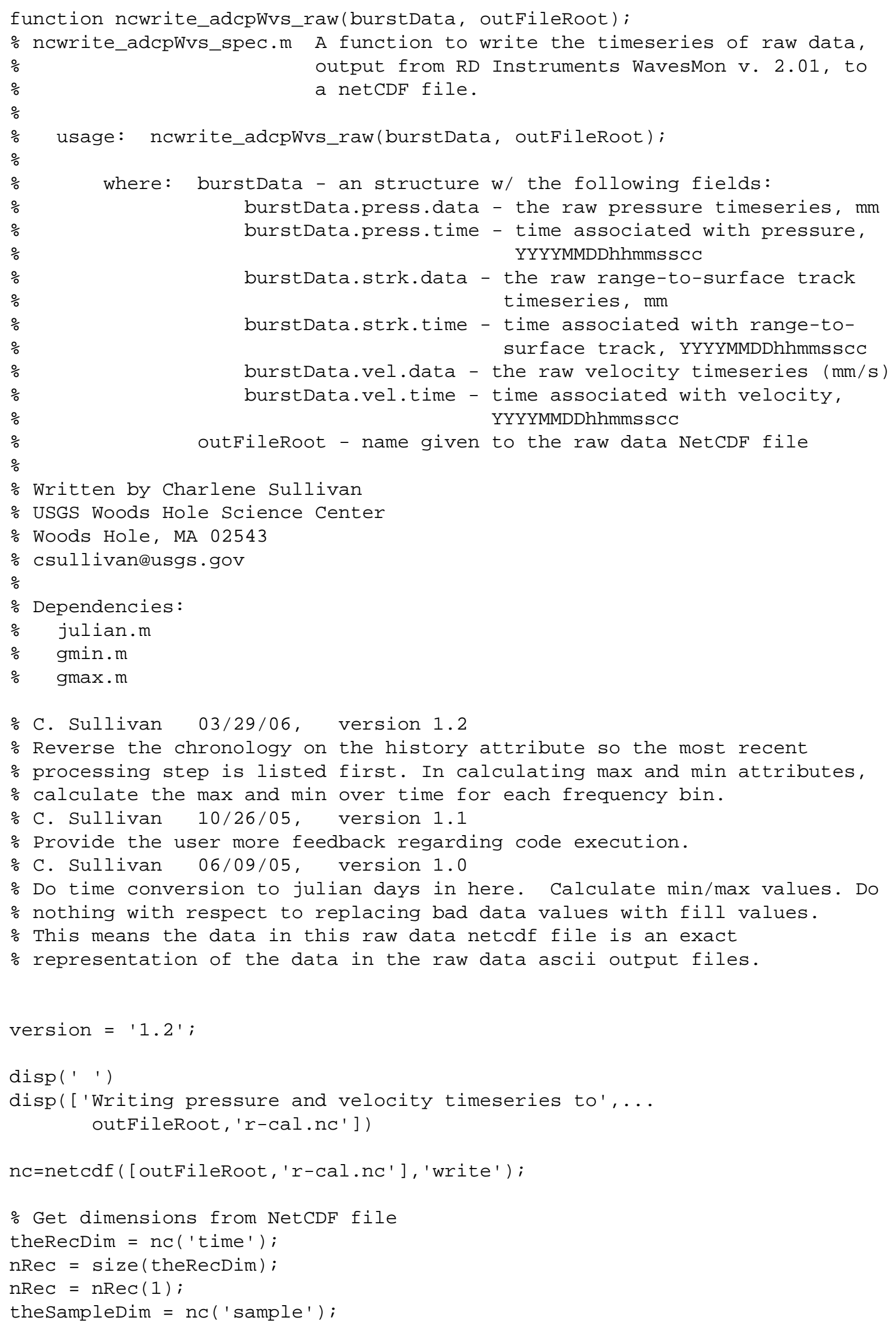




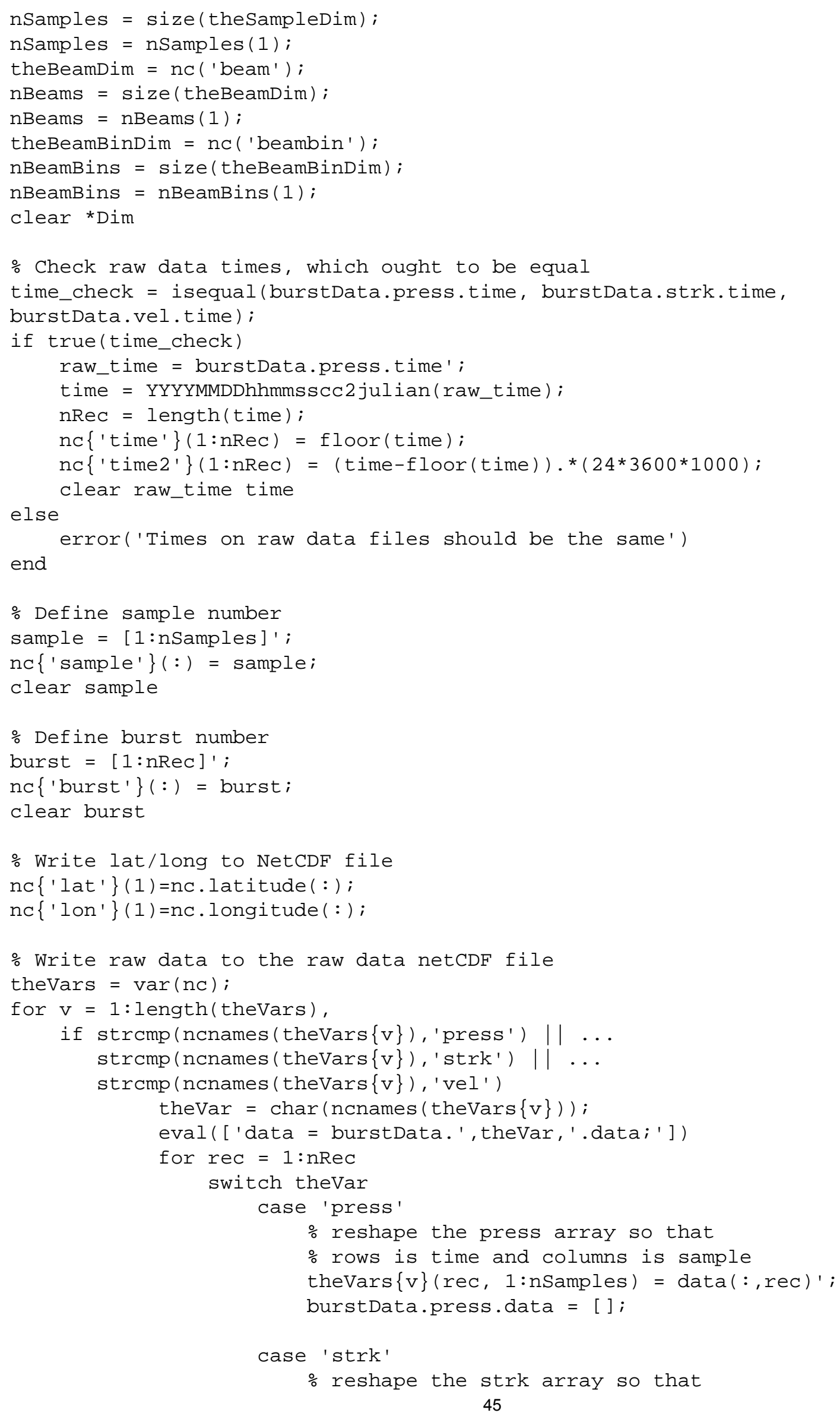


length (data $(:$, rec $)) /$ nBeams )' ;

$\%$ rows is time, columns is sample, and

$\%$ the $3 r d$ dimension is number of beams (4)

temp $=$ reshape $(\operatorname{data}(:$, rec $)$, nBeams,

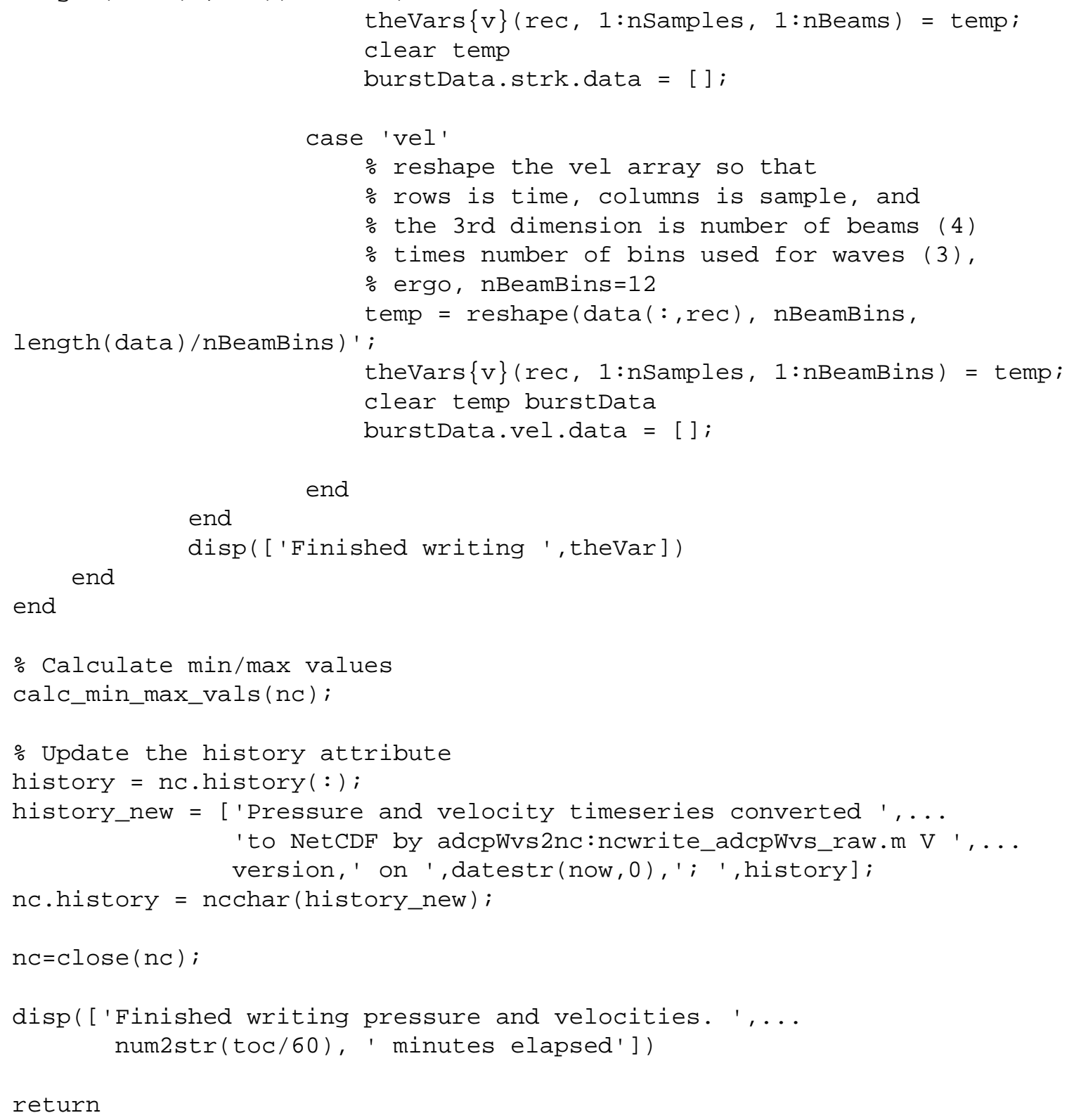




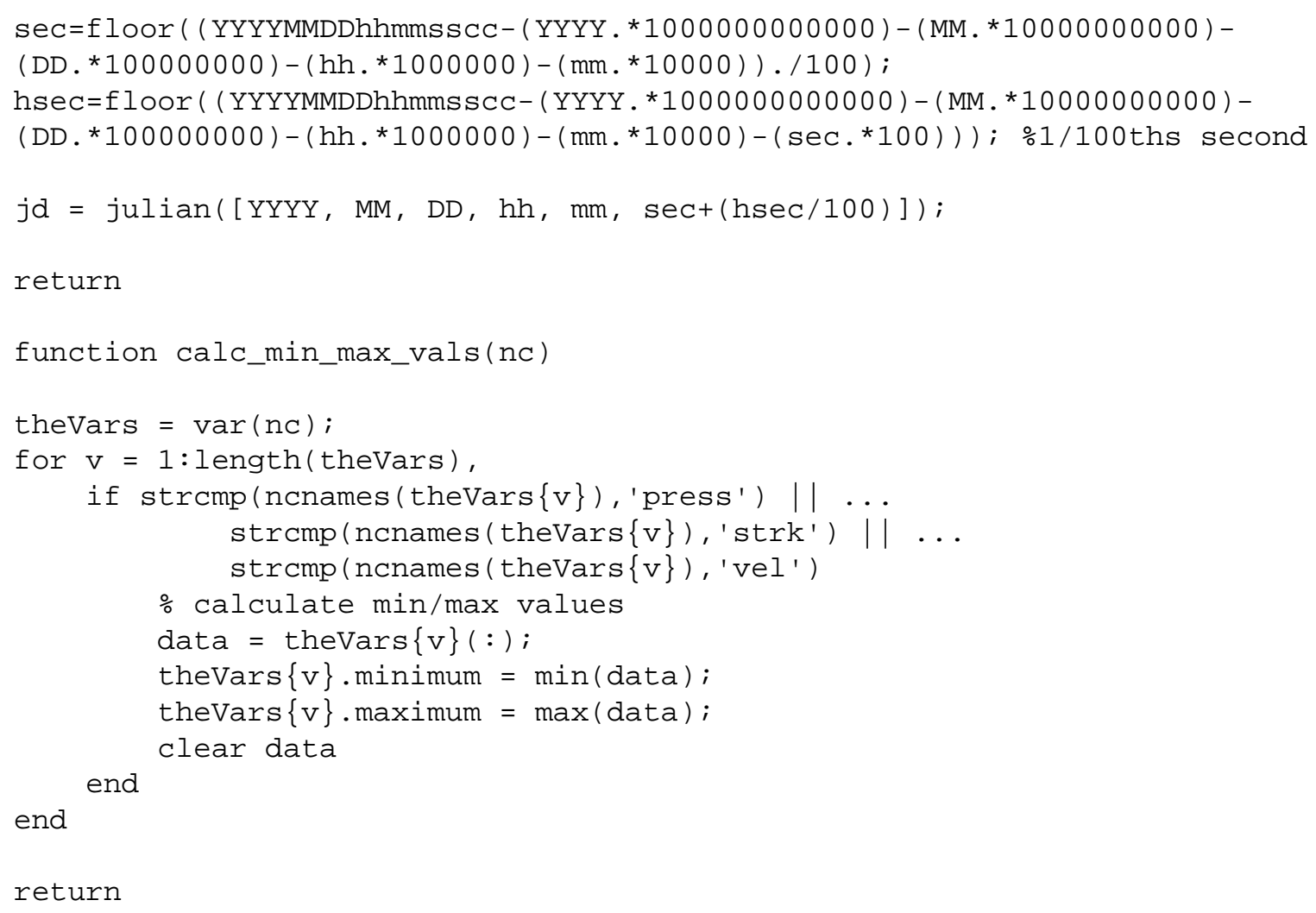




\section{Appendix V: M-files for Sontek Argonaut}

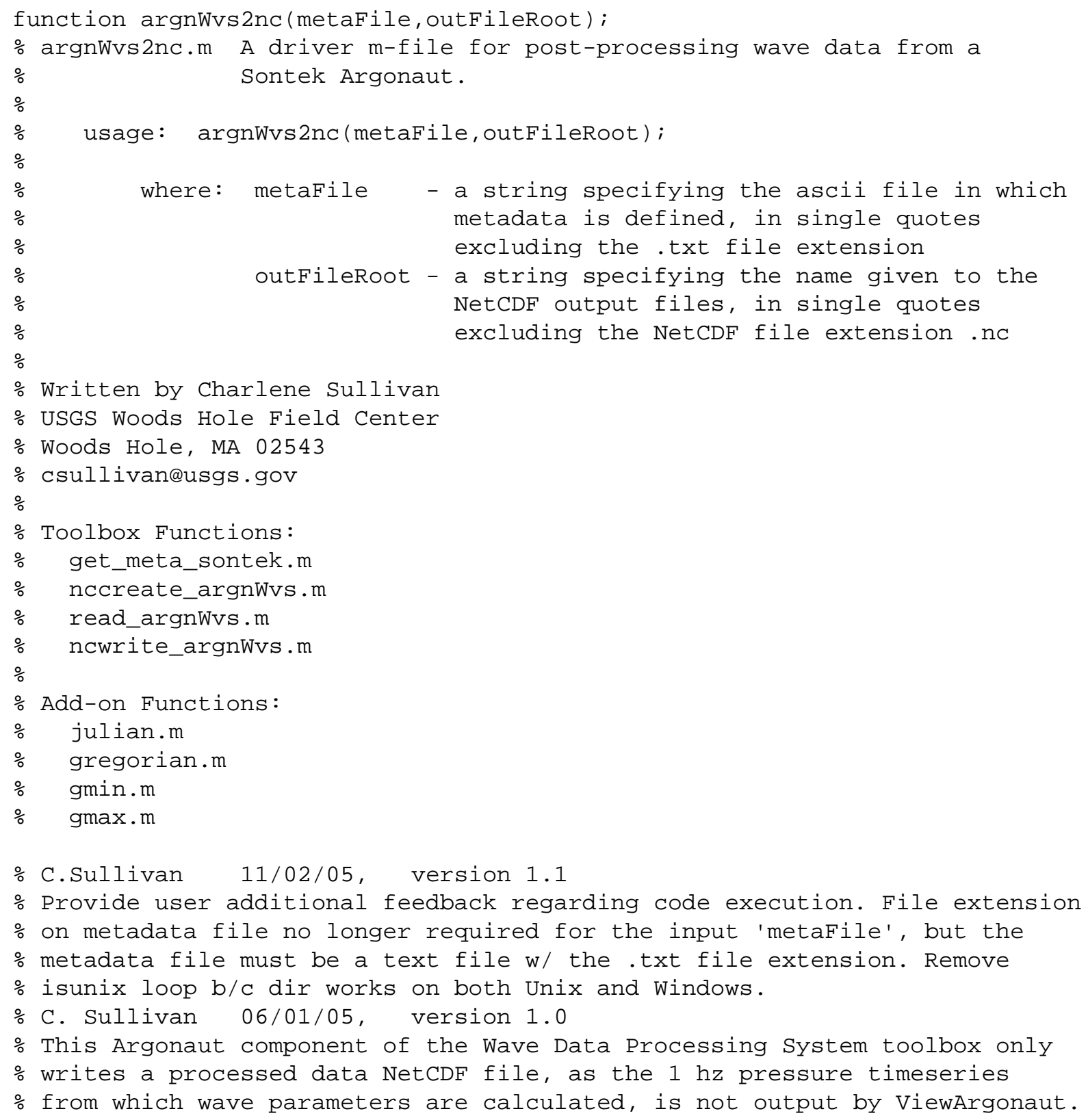




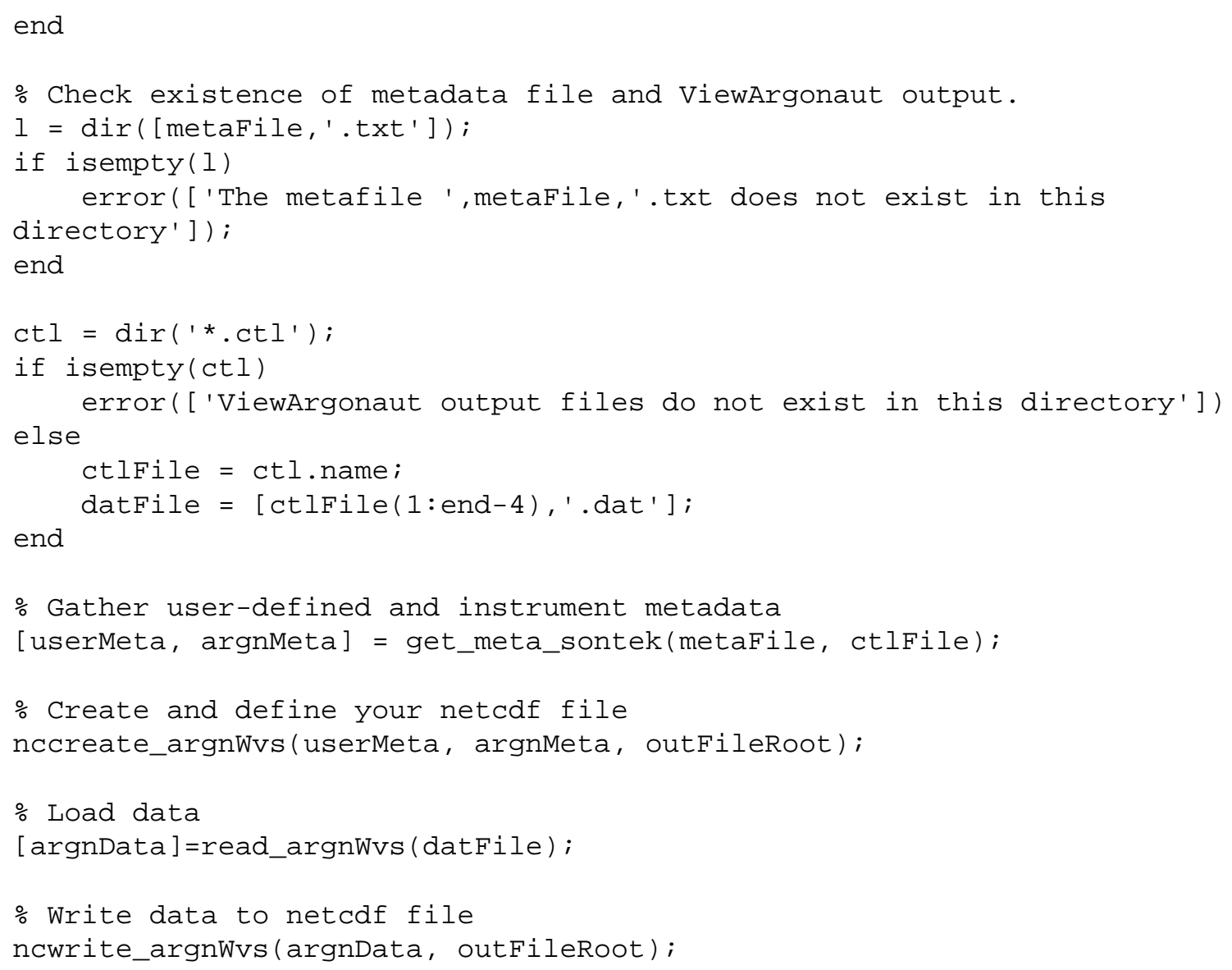




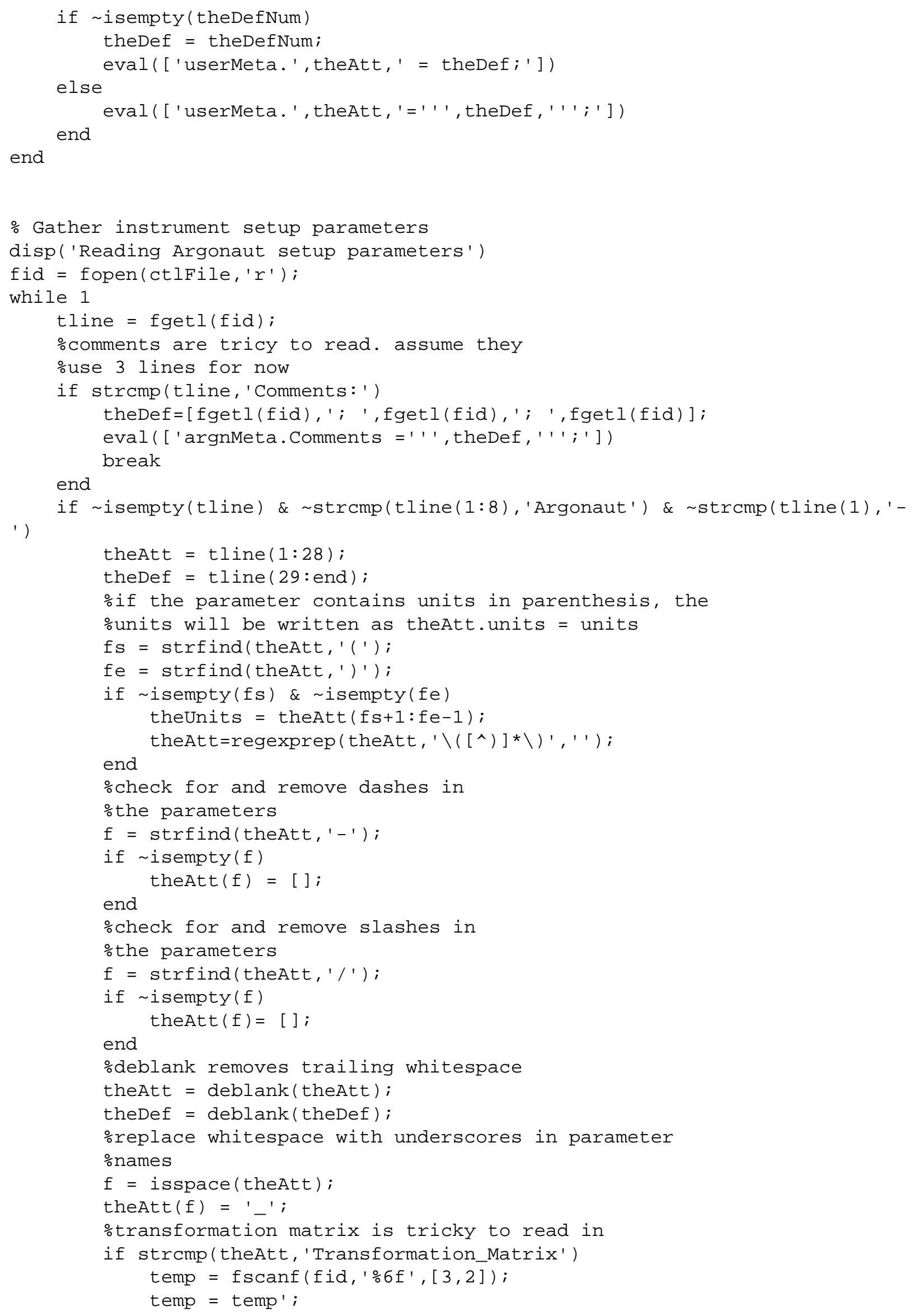




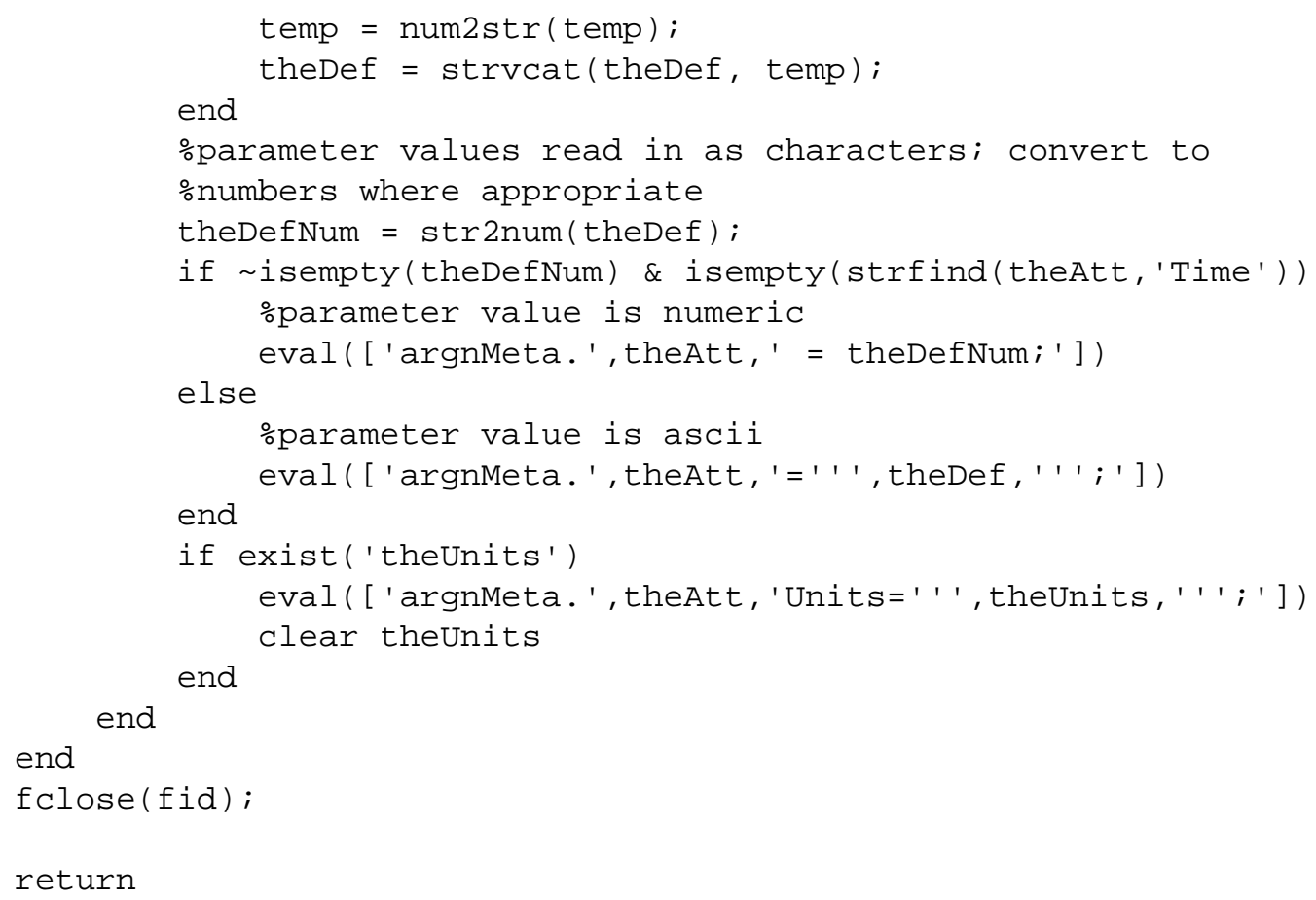

return 


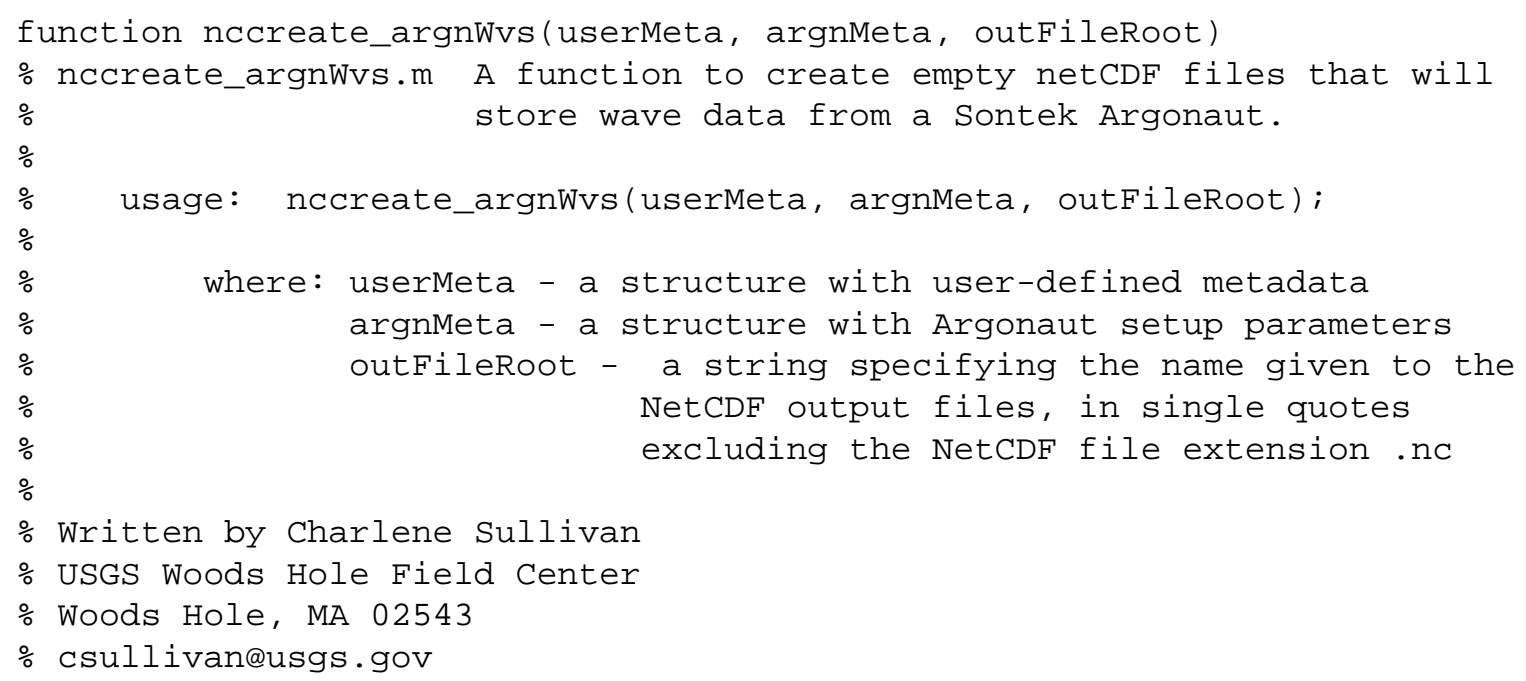

\% C. Sullivan $03 / 30 / 06$, version 1.2

\% Use EPIC keys 1239 and 4063 for the variables hght_std and wp_peak,

$\%$ respectively.

$\%$ C. Sullivan 11/02/05, version 1.1

$\%$ Provide the user additional feedback regarding code execution. Changed

$\%$ DATA_TYPE attribute description to be consistent w/ the documentation.

$\%$ Changed EPIC code and units on the variable lon to 502 and degree_east

$\%$ for consistency $w /$ longitude as specified in the metadata file where west

$\%$ is negative.

$\%$ C. Sullivan $06 / 01 / 05$, version 1.0

$\%$ Instrument setup information is now included in the metadata. only the

$\%$ processed data NetCDF file is created; the file includes wave parameters

$\%$ which were calculated from a $1 \mathrm{~Hz}$ pressure timeseries. No raw data

$\%$ NetCDF file is created as the $1 \mathrm{~Hz}$ pressure timeseries $\mathrm{s}$ not output by

$\%$ ViewArgonaut. It is assumed there are 10 frequency bands in the wave

$\%$ energy spectra.

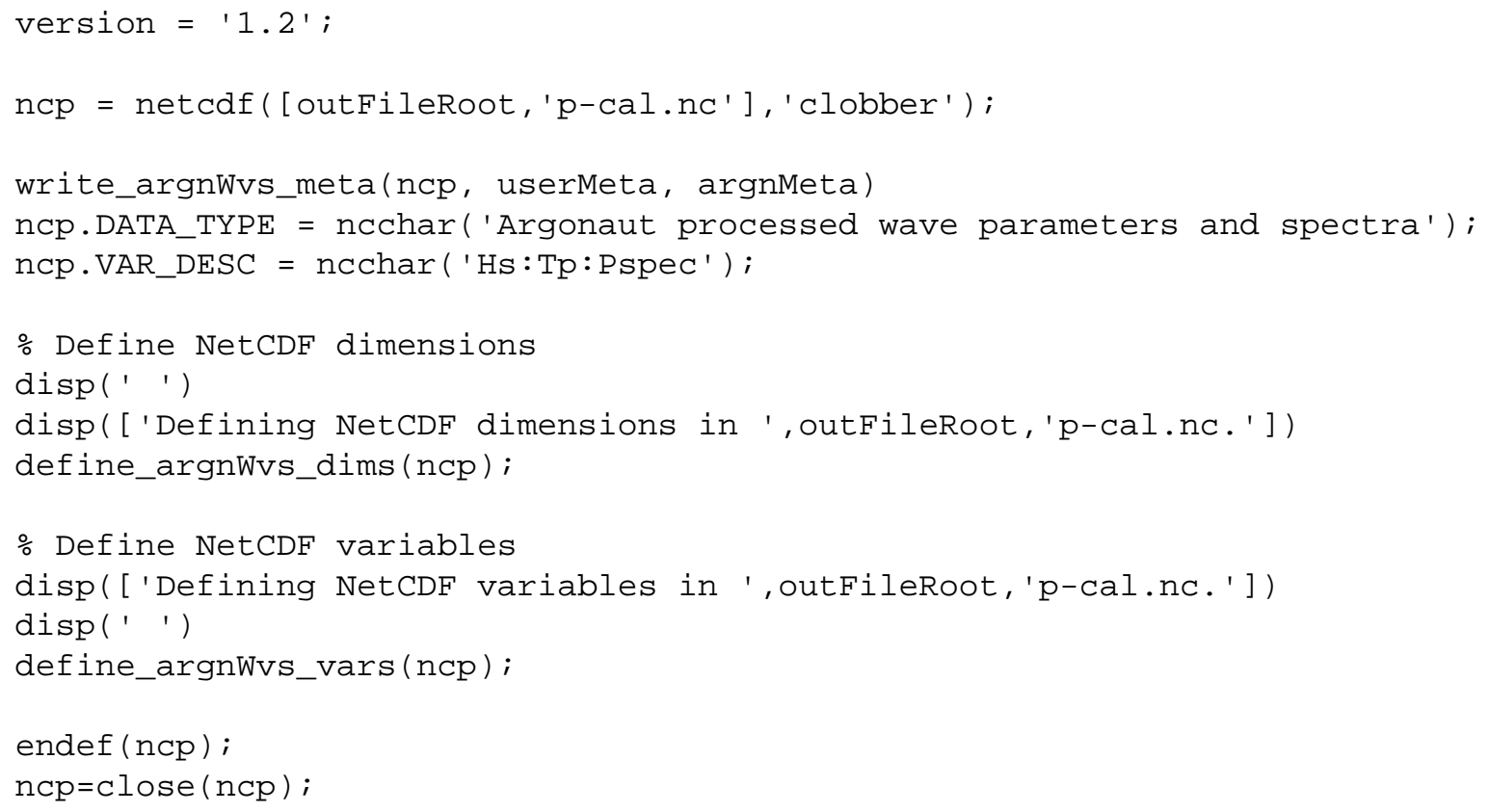


return

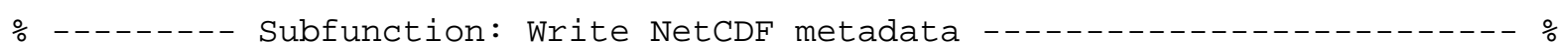
function write_argnWvs_meta(nc, userMeta, argnMeta)

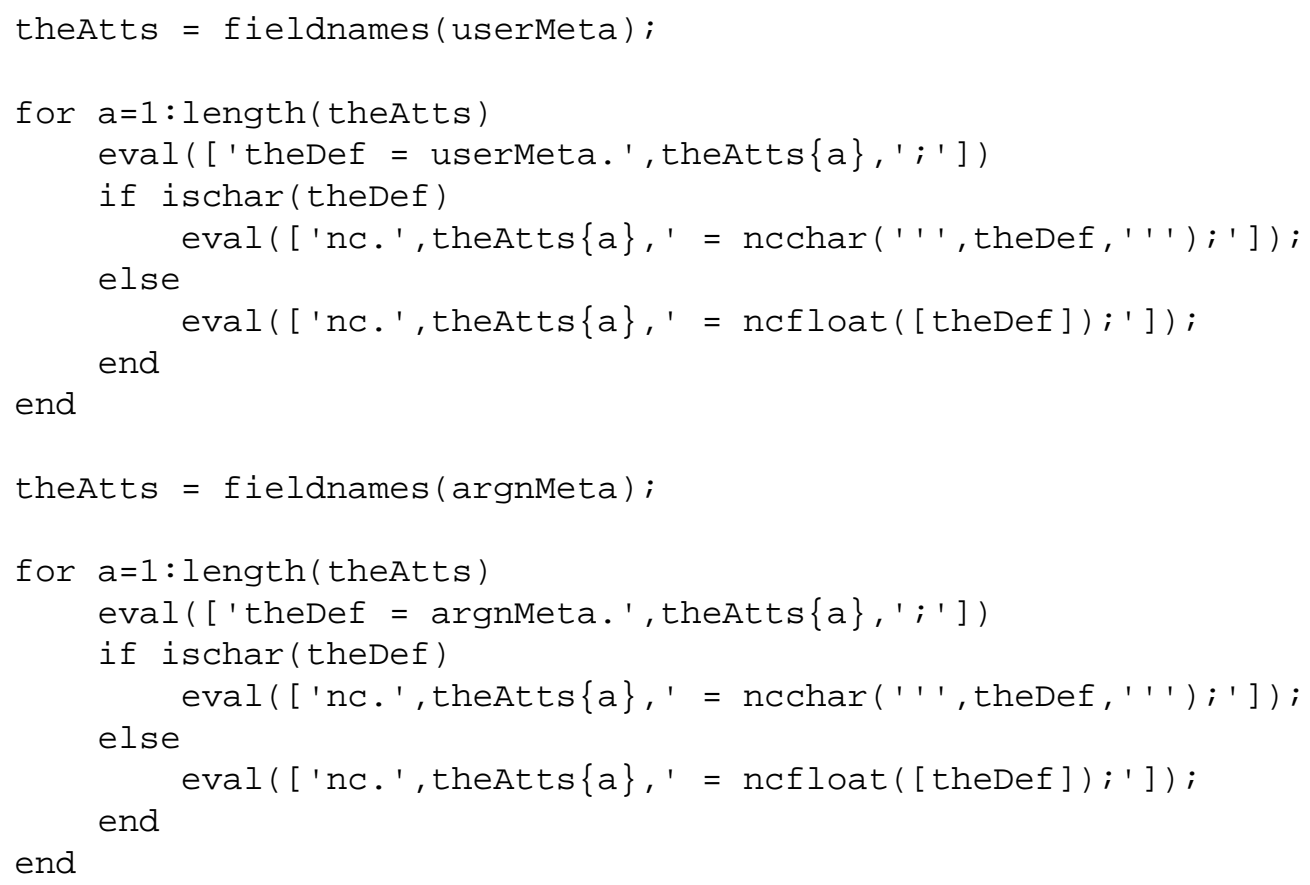




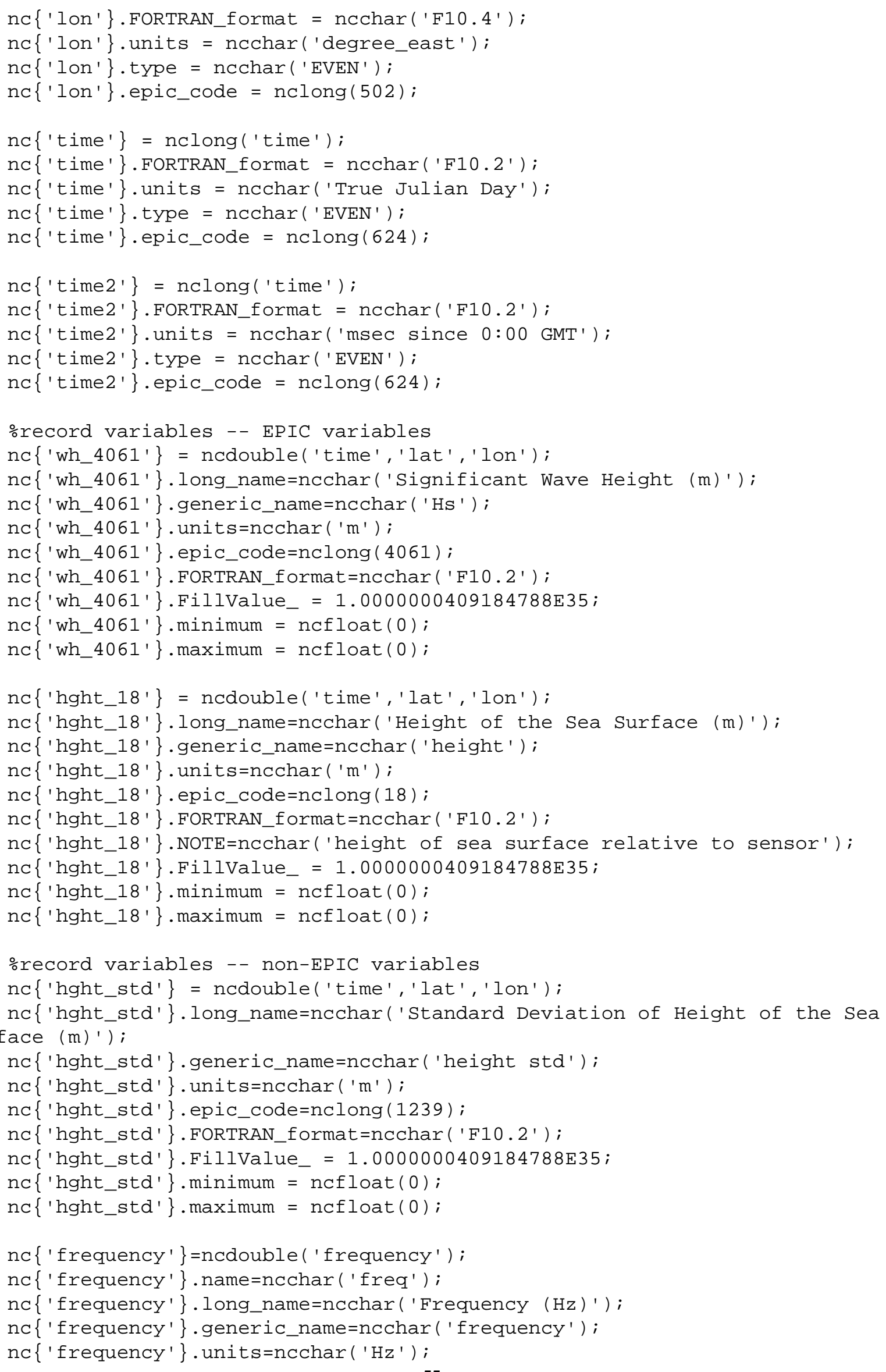


nc $\{$ 'frequency' $\}$.NOTE=ncchar ('frequency at the center of each frequency band ' );

nc $\{$ 'frequency' $\}$. FORTRAN_format=ncchar ( 'F10.2' );

nc $\{$ 'frequency' $\}$. minimum $=\operatorname{ncfloat}(\Theta)$;

$n c\{$ 'frequency' $\}$. maximum $=\operatorname{ncfloat}(\odot)$;

nc $\{$ 'wp_peak' $\}=$ ncdouble('time', 'lat', 'lon');

nc $\{$ 'wp_peak' $\}$. long_name=ncchar ('Peak wave Period (s)');

nc $\{$ 'wp_peak' $\}$.generic_name=ncchar ( 'Tp');

nc $\{$ 'wp_peak' $\}$. units=ncchar ('s');

nc $\{$ 'wp_peak' $\}$.epic_code=nclong (4063);

nc $\{$ 'wp_peak' $\}$. FORTRAN_format=ncchar ( 'F10.2' );

nc $\{$ 'wp_peak' $\}$. FillValue_ $=1.00000000409184788 \mathrm{E} 35$;

nc $\{$ 'wp_peak' $\}$. minimum $=\operatorname{ncfloat}(\Theta)$;

nc $\{$ 'wp_peak' $\}$. maximum $=\operatorname{ncfloat}(\Theta)$;

nc $\{$ 'pspec' $\}=$ ncfloat ('time' , 'frequency', 'lat', 'lon');

$\mathrm{nc}\{$ 'pspec' $\}$. name=ncchar $($ 'pspec');

nc $\{$ 'pspec' $\}$. long_name=ncchar ('Pressure-derived Non-directional Wave

Height Spectrum (mm/sqrt(Hz))');

nc $\{$ 'pspec'\} .generic_name=ncchar ('velocity spectrum');

nc $\{$ 'pspec' $\}$. units=ncchar ('mm/sqrt $(\mathrm{Hz})$ ') ;

nc $\{$ 'pspec' $\}$. FORTRAN_format=ncchar ( 'F10.2');

nc $\{$ 'pspec' $\}$. FillValue $=1.0000000409184788 \mathrm{E} 35$;

$\mathrm{nc}\{$ 'pspec' $\} \cdot \operatorname{minimum}=\operatorname{ncfloat}(\Theta)$;

$\mathrm{nc}\{$ 'pspec' $\} \cdot \operatorname{maximum}=\operatorname{ncfloat}(\odot)$;

return 


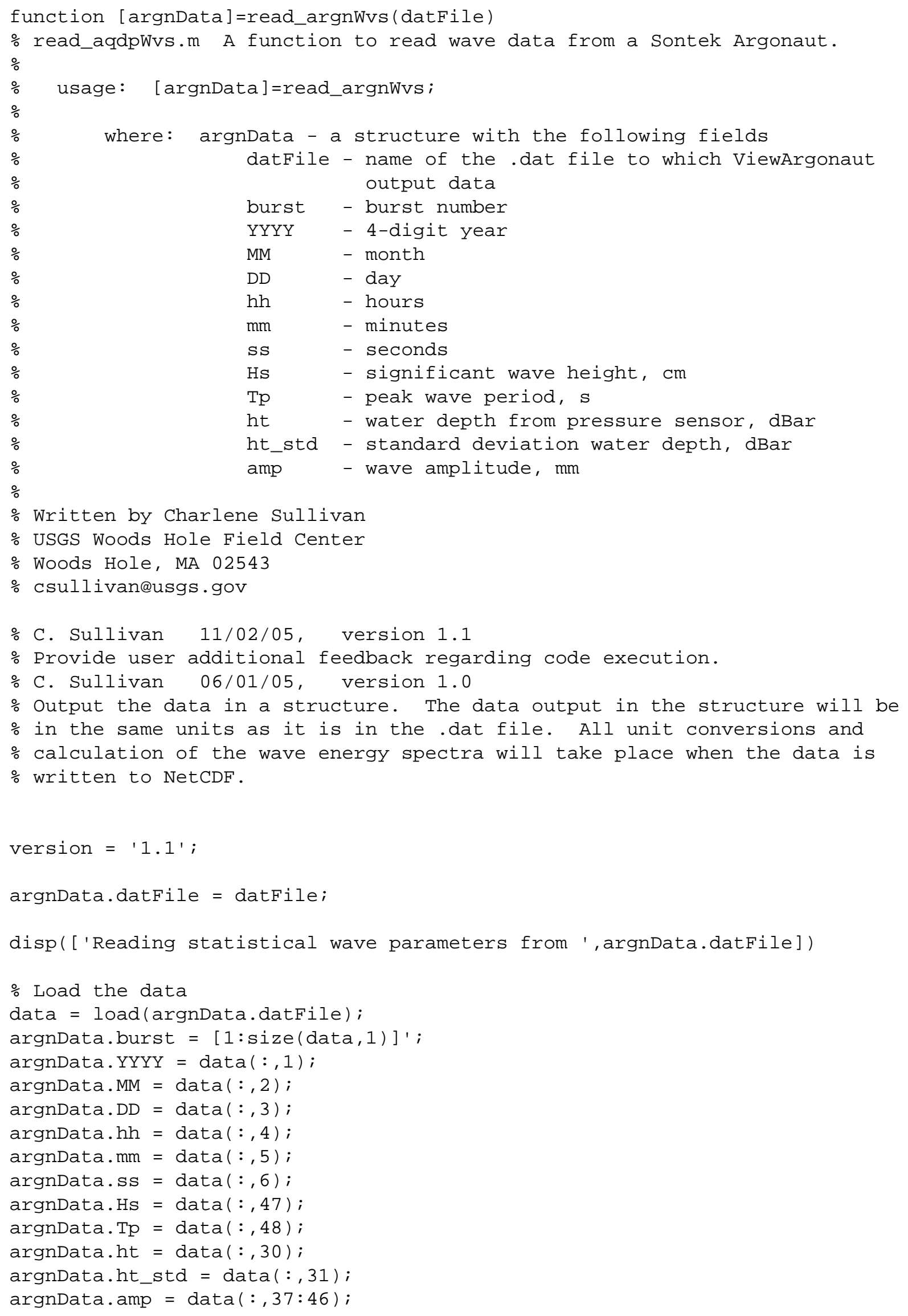


return 
function ncwrite_argnWvs(argnData, outFileRoot)

$\%$ ncwrite_adcpwvs.m A function to write wave data from a Sontek Argonaut. $\%$

usage: ncwrite_aqdpWvs(argnData, outFileRoot);

where: argnData - a structure with the following fields

datFile - name of the . dat file to which ViewArgonaut output data

burst - burst number

YYYY - 4-digit year

MM $\quad-$ month

DD - day

hh $\quad-$ hours

$\mathrm{mm} \quad-$ minutes

ss - seconds

Hs - significant wave height, $\mathrm{cm}$

Tp - peak wave period, $s$

ht - water depth from pressure sensor, dBar

ht_std - standard deviation water depth, dBar

amp - wave amplitude, $\mathrm{mm}$

outFileRoot - a string specifying the name given to the NetCDF output files, in single quotes excluding the NetCDF file extension .nc

Written by Charlene Sullivan

USGS Woods Hole Field Center

Woods Hole, MA 02543

csullivan@usgs.gov

Dependencies:

julian.m

gregorian.m

gmin.m

$\operatorname{gmax} \cdot \mathrm{m}$

\% C.Sullivan $03 / 30 / 06$, version 1.3

$\%$ Reverse the chronology on the history attribute so the most recent

$\%$ processing step is listed first. For max and min attributes on the

$\%$ variable pspec, calculate the maxs and mins over time for each frequency

$\%$ band.

$\%$ C.Sullivan 11/02/05, version 1.2

$\%$ Provide the user additional feedback regarding code execution.

$\%$ C. Sullivan 06/09/05, version 1.1

$\%$ Users is interactively asked to view a plot to determine which bursts are $\%$ good. Only good bursts are written to NetCDF.

$\%$ C. Sullivan 06/01/05, version 1.0

$\%$ Unit conversions and the calculation of wave spectra take place in this $\% \mathrm{~m}$-file. Data is written to the processed data NetCDF file. No raw data $\%$ NetCDF file is produced as the $1 \mathrm{~Hz}$ pressure timeseries from which wave $\%$ parameters are calculated is not output by ViewArgonaut.

version $=' 1.2$ ';

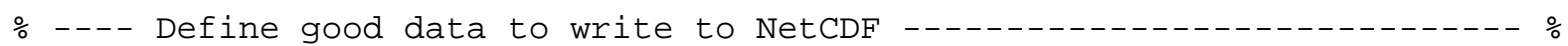
\%display a figure with two subplots. the first subplot has mean pressure \%and the standard deviation of pressure. the second subplot contains the 
\%significant wave height, and the peak wave period. the user is asked to $\%$ view these plots (zooming in and out) and determine the first and last \%good bursts of data. all data between the first and last $\%$ good data points will be written to NetCDF.

$\mathrm{F}=$ figure;

set(F,'position', [[200 100800800$]$ )

subplot (411)

plot (argnData.burst, argnData.ht)

ylabel( \{'Pressure', '(dBar)'\})

subplot (412)

plot (argnData.burst, argnData.ht_std)

ylabel(\{'Pressure Standard', 'Deviation (dBar)'\})

subplot (413)

plot(argnData.burst, argnData.Hs)

ylabel(\{'Significant', 'Wave Height $(\mathrm{cm})$ '\})

subplot (414)

plot (argnData.burst, argnData.Tp)

ylabel( $\{$ 'Peak Wave', 'Period (s)' $\}$ )

xlabel( 'Index' )

$\operatorname{disp}('$ ' ')

disp('Please view the figure (feel free to zoom!) and look for indices which might be bad')

disp('Bad indices might include out-of-water indices, for example')

answer $=$ input $([$ 'Would you like to write all indices (indices 1:', ... num2str(length(argnData.burst)),') to NetCDF? y/n : '], 's');

if strcmp (answer, ' $Y$ ') \& strcmp(answer, ' $y$ ') \& ...

-strcmp (answer, 'N') \& strcmp(answer, ' $n$ ')

disp(['Valid answers are either yes ("y") or no ("n")'])

answer $=$ input (['Would you like to write all indices (indices 1:', ..

'], 's'); num2str(length(argnData.burst)),' ) to NetCDF? $\mathrm{y} / \mathrm{n}$ :

end

if strcmp(answer, ' $Y$ ') || strcmp(answer, ' $y$ ')

first_good = 1;

last_good = length(argnData $\cdot$ burst);

disp(['Including indices 1 through ', num2str(length(argnData.burst)), ' in

PUV analysis'])

elseif strcmp(answer, 'N') || strcmp(answer, 'n')

first_good $=$ input('Please view the figure and enter the first good

indice: ');

if isempty(first_good)

disp('Valid answers are numeric only')

first_good $=$ input('Please view the figure and enter the first good

indice: ');

end

last_good $=$ input ('Please view the figure and enter the last good indice: ');

if isempty(last_good)

disp('Valid answers are numeric only')

last_good $=$ input ('Please view the figure and enter the last good

indice: ');

end

disp(['Writing indices ', num2str(first_good),' to ', num2str(last_good), .

' to NetCDF']) 


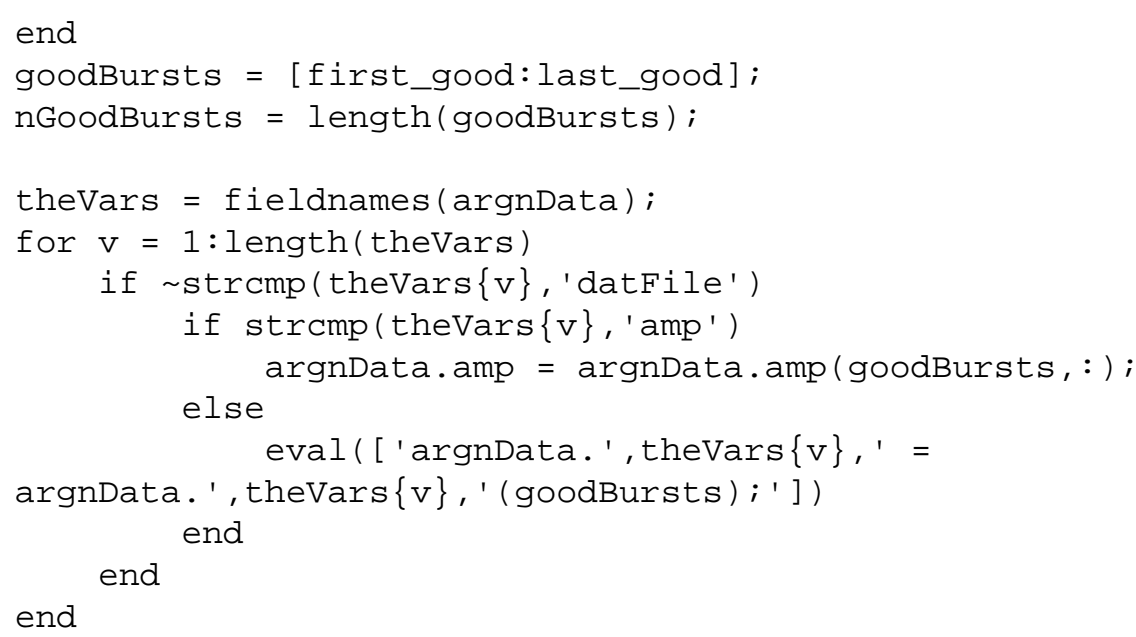




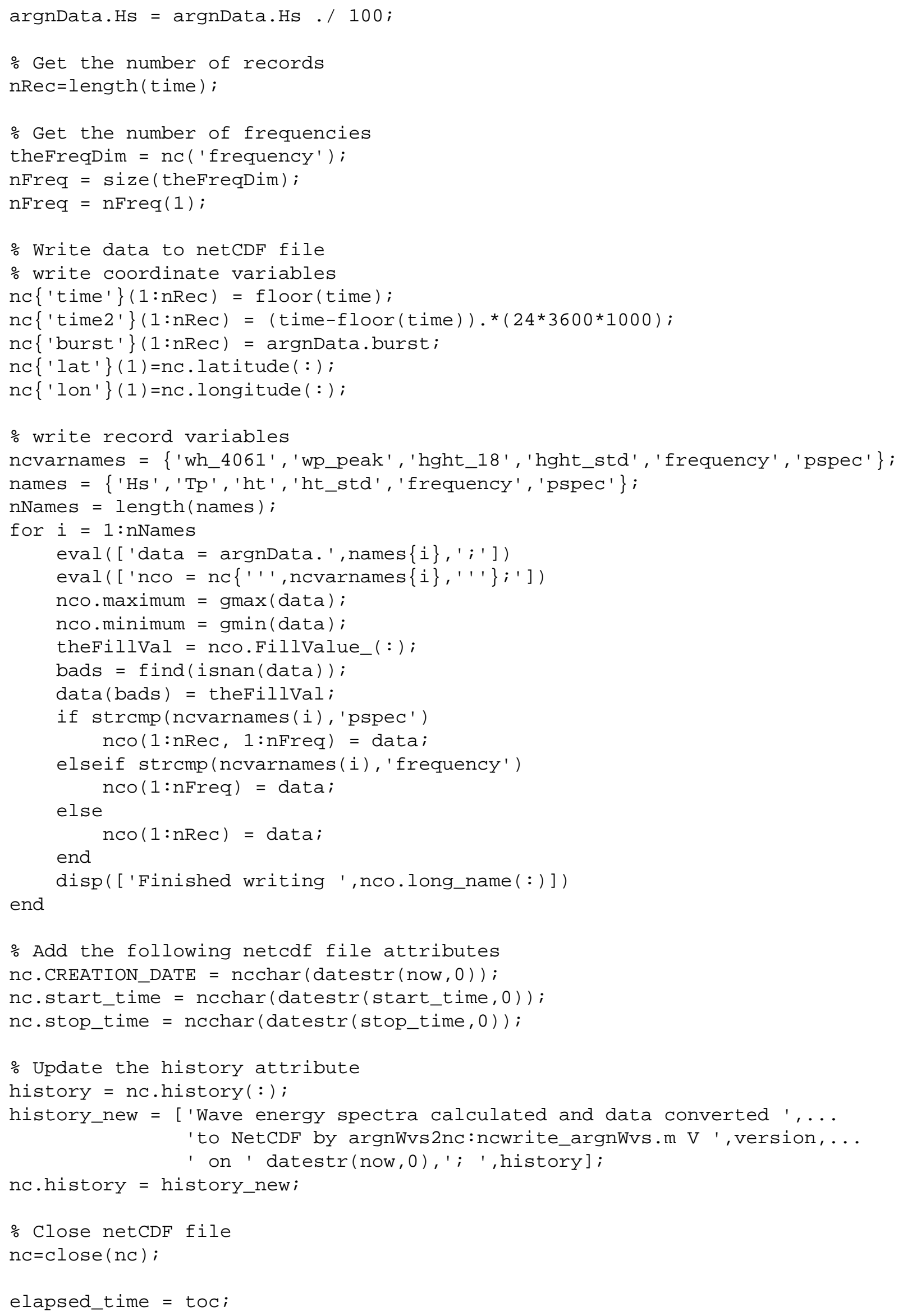


disp(['Finished writing statistical wave parameters and spectra. ',... num2str(toc/60),' minutes elapsed.'])

return 


\section{Appendix VI: M-files for Nortek AP}

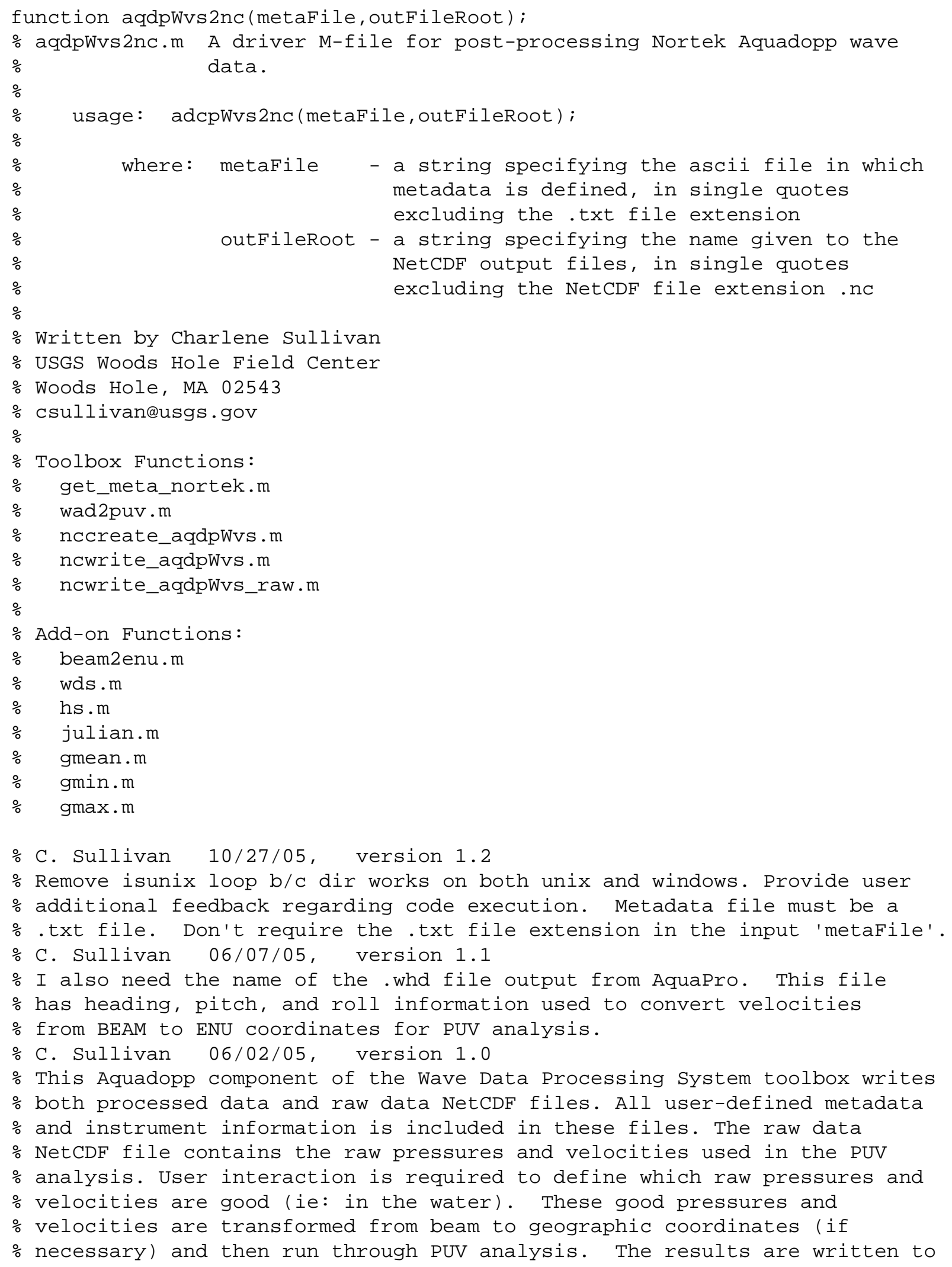


$\%$ the processed data NetCDF file. Therefore, out-of-water data is excluded $\%$ from PUV analysis and the processed data NetCDF file.

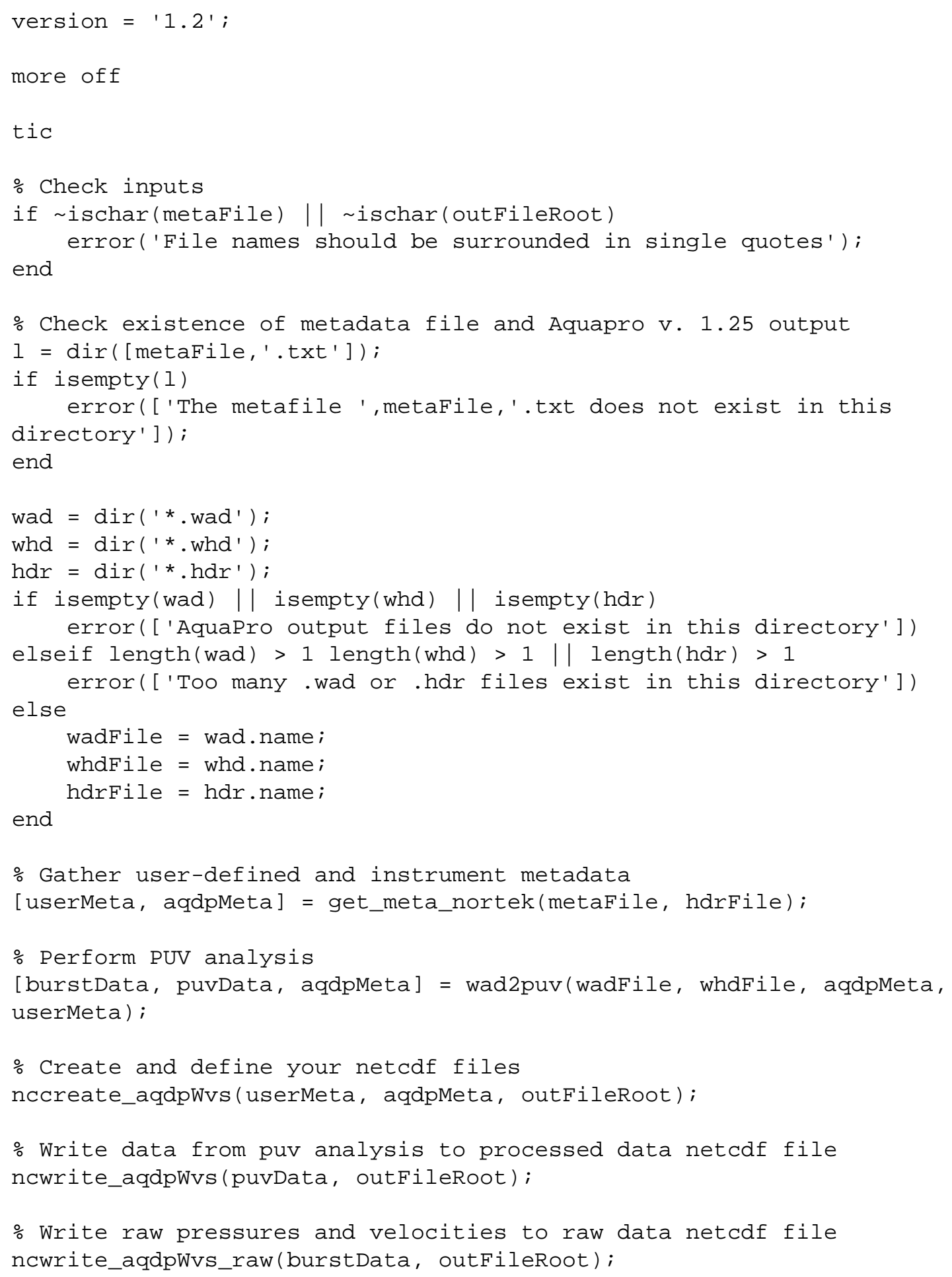




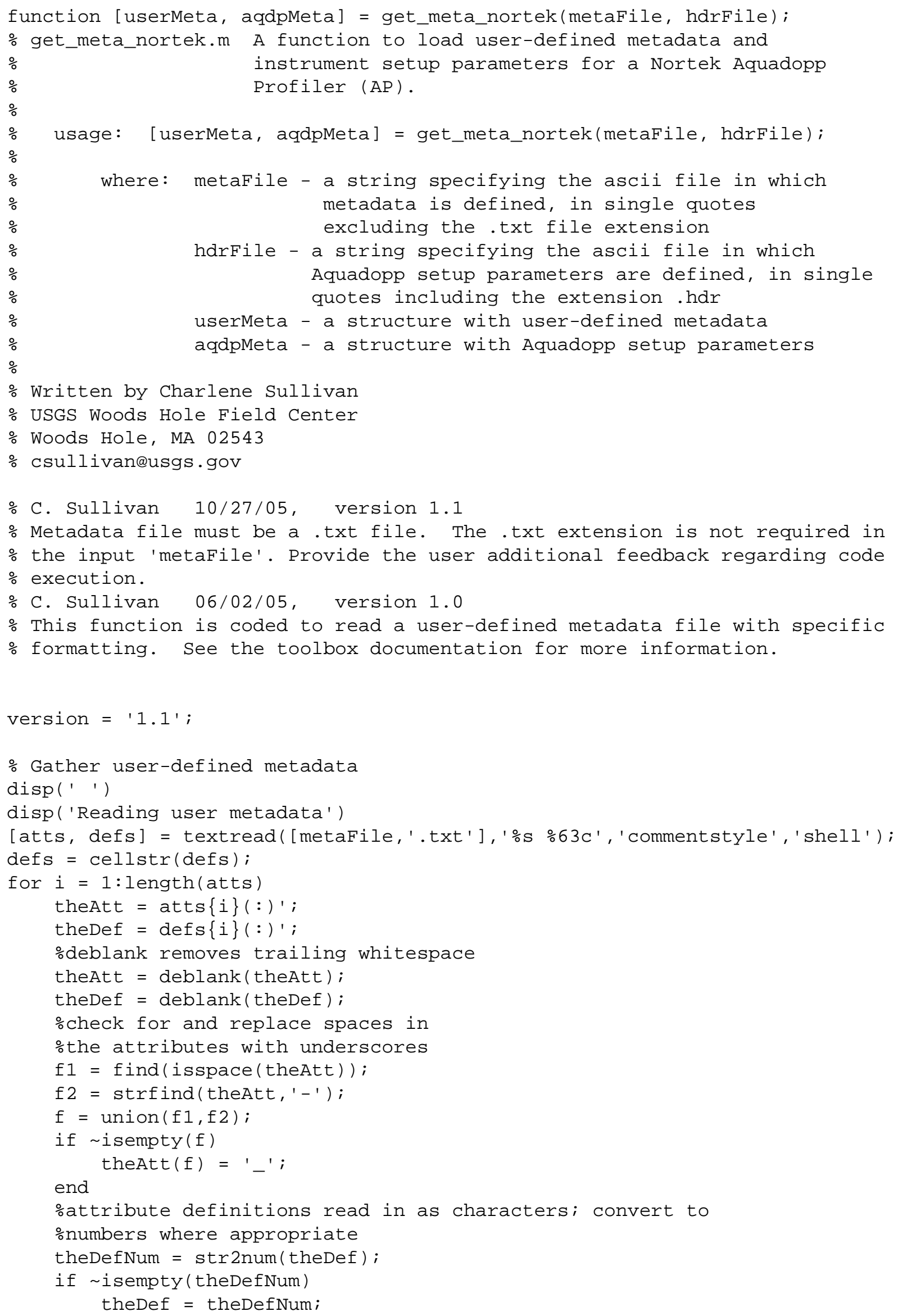




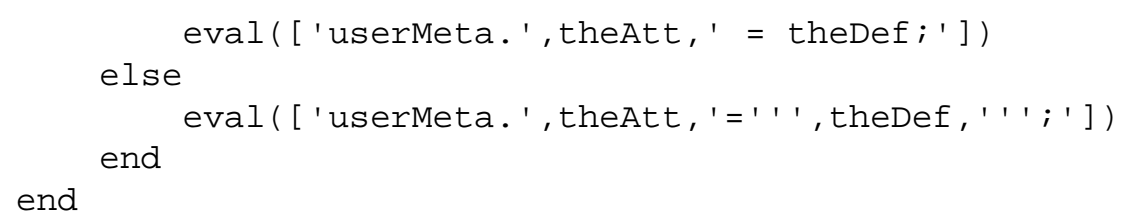




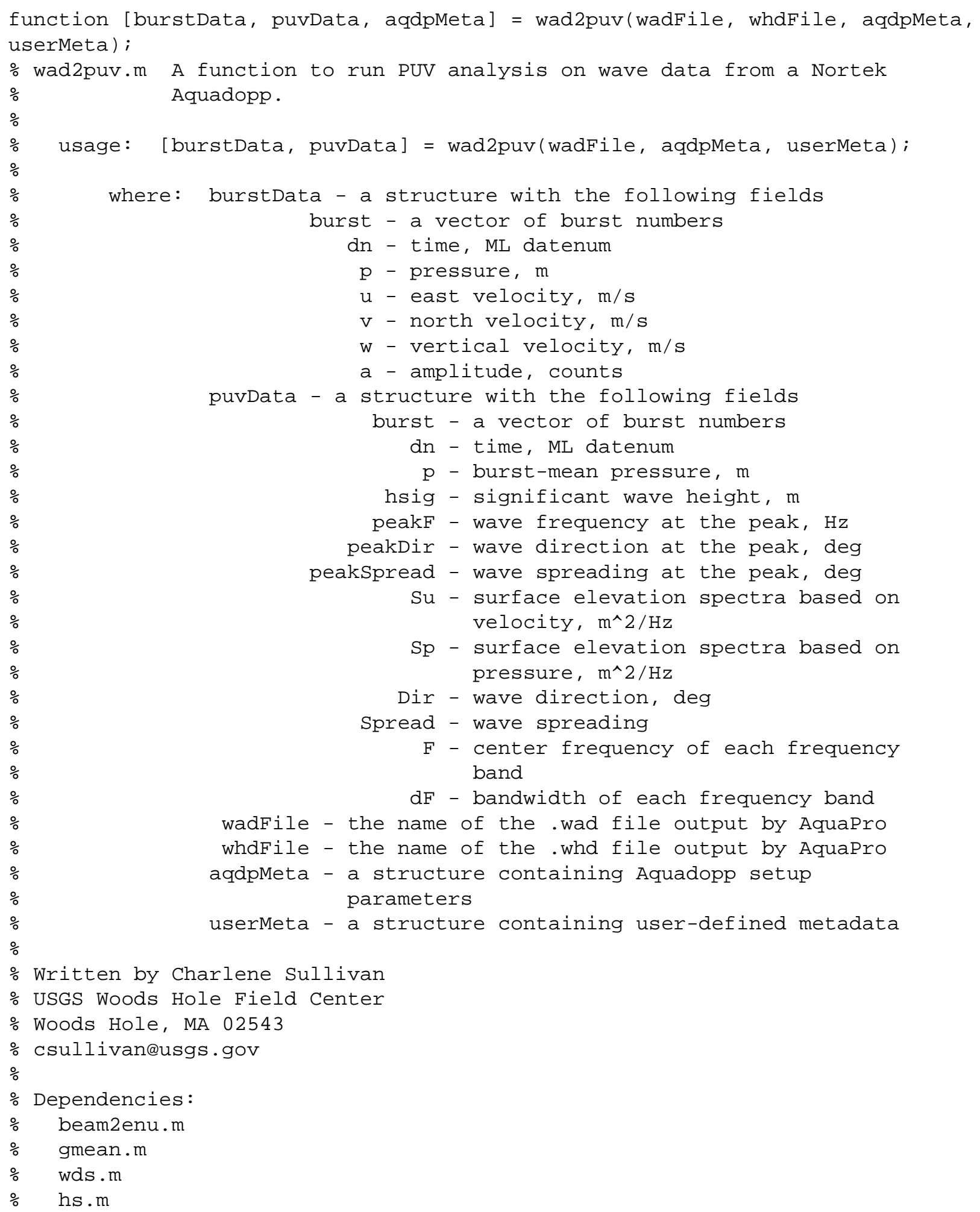

$\%$ C. Sullivan 10/27/05, version 1.2

$\%$ Provide user additional feedback regarding code execution. Add version

$\%$ number.

$\%$ C. Sullivan $06 / 06 / 05$, version 1.1

$\%$ Velocities collected in beam coordinates must be converted to geographic

$\%$ coordinates prior to PUV analysis. Alert user that PUV parameters in

$\%$ user-defined metadata file are being applied. If user enters an erroneous 
$\%$ input (anything non-numeric including carriage return) for either $\%$ 'first_good' or 'last_good', they are now asked to re-enter their answers. $\%$ C. Sullivan $06 / 02 / 05$, version 1.0

$\%$ This function combines elements of George Voulgaris' function split_wad.m $\%$ and puv_nortek.m. The idea is to read the Aquadopp's . wad file, split the $\%$ pressure and velocity data into individual bursts, and perform PUV $\%$ analysis burst by burst for only good bursts. It is assumed colums 8, 9, $\%$ and 17 in the .wad file are empty. The pressure and velocity data is $\%$ stored in arrays that are dimensioned [nSamples $x$ nBursts]. The user is $\%$ asked which bursts to perform the PUV analysis on. The mfiles for the PUV $\%$ analysis are wds.m and hs.m, which were provided by Nortek. The data from $\%$ PUV analysis is stored in arrays that are dimensioned [ 1 x nGoodBursts] $\%$ for wave parameters and [nFreq $x$ nGoodBursts] for wave spectra.

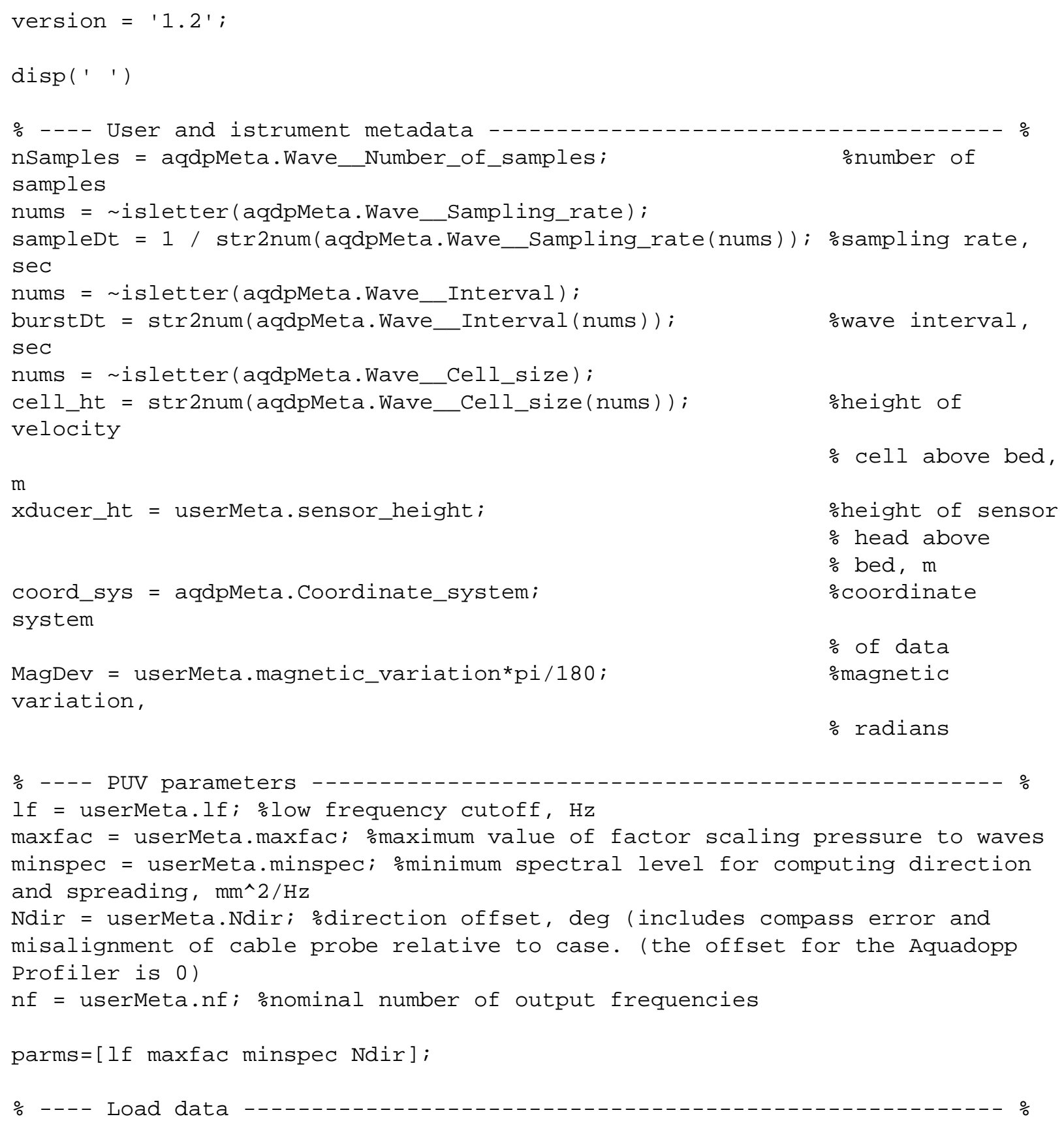


disp(['Loading Aquadopp pressure and velocity data from ', wadFile, ... '. This may take a few minutes.'])

data $=\operatorname{load}($ wadFile $)$;

mo $=\operatorname{data}(:, 1)$;

day $=\operatorname{data}(:, 2)$;

yy $=\operatorname{data}(:, 3)$;

$\mathrm{hh}=\operatorname{data}(:, 4)$;

$\mathrm{mi}=\operatorname{data}(:, 5)$;

ss $=\operatorname{data}(:, 6)$;

$\mathrm{p}=\operatorname{data}(:, 7) ; \quad \%$ meters

$\mathrm{v} 1=\operatorname{data}(:, 10) ; \%$ beam1 $|\mathrm{X}|$ East velocity $(\mathrm{m} / \mathrm{s})$

$\mathrm{v} 2=\operatorname{data}(:, 11) ; \%$ beam2 $|\mathrm{Y}| \operatorname{North}$ velocity $(\mathrm{m} / \mathrm{s})$

$\mathrm{v} 3=\operatorname{data}(:, 12) ; \%$ beam3 $\mid$ Z|UP velocity $(\mathrm{m} / \mathrm{s})$

a1 $=\operatorname{data}(:, 14) ;$ \%Amplitude Beam 1 (counts)

a2 $=\operatorname{data}(:, 15) ;$ \%Amplitude Beam 2 (counts)

a3 $=\operatorname{data}(:, 16) ; \%$ Amplitude Beam 3 (counts)

data $=\operatorname{load}($ whdFile $) ;$

hdg $=\operatorname{data}(:, 12)$;

ptch $=\operatorname{data}(:, 13)$;

roll = data $(:, 14)$;

\%we use a time-based method for defining bursts in case the $\#$ of bursts

\%recorded to the wad file does not equal the length(.wad file)/nSamples dno $=$ datenum $(y y$, mo, day, hh, mi, ss $) ;$ \%time stamp on samples, ML datenum dnoff $=$ datenum $(\operatorname{yy}(1), 1,1,0,0,0)$;

$\mathrm{dn}=\mathrm{dno}-$ dnoff; $\quad \%<--$ Why is this done?

clear nums data mo day yy hh mi ss

\%

\%with a time-based method for defining bursts, the time between

\%bursts should be > (burstDt - (nSamples*sampleDt)). this is only valid

\%when (nSamples*sampleDt) < burstDt

time_btw_bursts $=($ burstDt $-($ nSamples * sampleDt $)) /(3600 * 24) ;$ \%days

\%Istart (a vector) indicates the indices in the wad file

\%at which bursts begin. however be sure to include $\odot$ as the

\%first value in Istart or else you will miss the first burst!

burst_start_ind $=$ find $(\operatorname{diff}(\operatorname{dn})>$ time_btw_bursts $) ; \%<--$ - why not just use dno?

burst_start_ind $=[0$; burst_start_ind $]$;

\%number of bursts is equal to the length of burst_start_ind

nBursts = length(burst_start_ind);

\%sometimes instrument is stopped during a burst

if length $(\mathrm{dn})<$ (burst_start_ind(end)+nSamples)

end

nBursts = nBursts -1 ;

\%pre-allocate the structure burstData. pressure, velocities, amplitudes, \%and dates will be written to this structure as fields that are

\%dimensioned [nSamples $x$ nBursts]

burstData. burst $=$ nan*ones $(1$, nBursts $)$; 


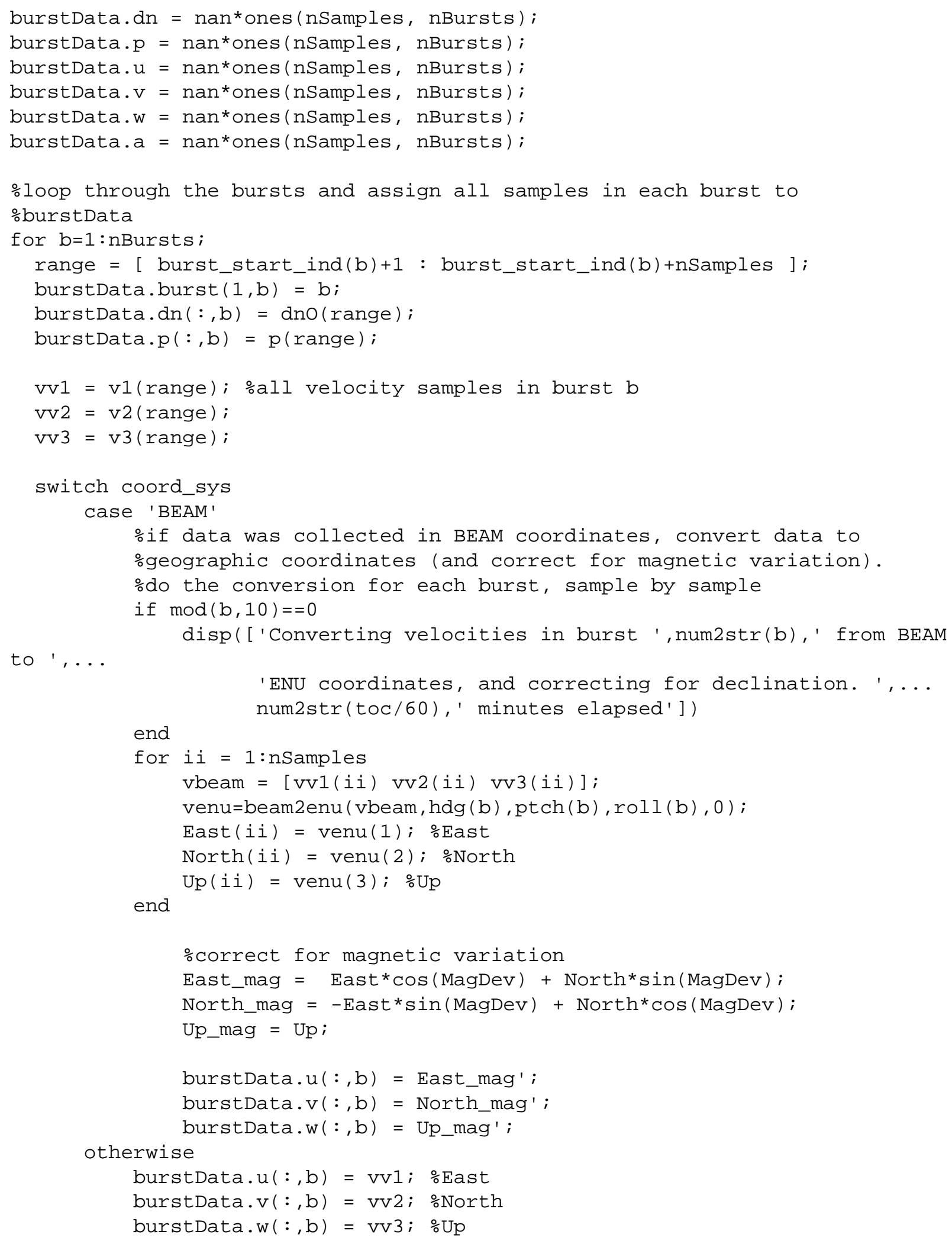

end

burstData.a $(:, b)=a 1($ range $)+a 2($ range $)+a 3($ range $) ;$

end 
\%clear variables you no longer need

clear p v1 v2 v3 a1 a2 a3

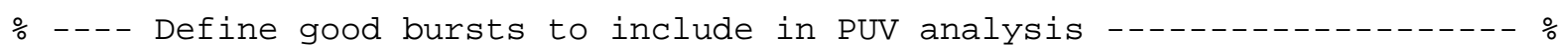
\%display a figure with burst-averaged pressure and velocities. ask the \%user if, based on the figure, they would like to include all bursts in the \%PUV analysis. if the answer is no, ask the user to enter the the first \%and last good bursts. all bursts between the first and last good bursts \%will be included in PUV analysis.

figure subplot (211) plot(burstData.burst, gmean(burstData.p))

ylabel('Burst-mean pressure (m)')

xlabel('Burst number')

subplot (212)

plot(burstData.burst, gmean(burstData.u))

hold on

plot (burstData.burst, gmean(burstData.v), ' $r$ ')

plot (burstData.burst, gmean(burstData.w), 'g')

ylabel('Burst-mean Velocity (m/s)')

xlabel ('Burst number')

legend('Eastward Velocity', 'Northward Velocity', 'Vertical Velocity')

disp(' ')

disp('Please view the figure (feel free to zoom!) and look for bursts which might be bad')

disp('Bad bursts might include out-of-water bursts, for example.')

answer = input (['Would you like to include all bursts (bursts 1:', ... num2str(nBursts),' ) in PUV analysis? y/n : '], 's');

if strcmp (answer, ' $Y$ ') \& strcmp (answer, ' $y$ ') \& ...

- strcmp (answer, 'N') \& strcmp (answer, 'n')

disp(['Valid answers are either yes ("y") or no ("n")'])

answer = input (['Would you like to include all bursts (bursts 1:', .. num2str(nBursts),') in PUV analysis? y/n : '],'s');

end

if strcmp(answer, ' $Y$ ') || strcmp(answer, ' $y$ ')

first_good = 1;

last_good $=$ nBursts;

disp(['Including bursts 1 through ', num2str(nBursts),' in PUV analysis'])

elseif strcmp(answer, 'N') || strcmp(answer, 'n')

first_good $=$ input('Please view the figure and enter the first good burst: ');

if isempty(first_good)

disp('Valid answers are numeric only')

first_good $=$ input('Please view the figure and enter the first good

burst: ');

end

last_good $=$ input $($ 'Please view the figure and enter the last good burst:

');

if isempty(last_good)

disp('Valid answers are numeric only')

last_good = input('Please view the figure and enter the last good

burst: ' );

end 


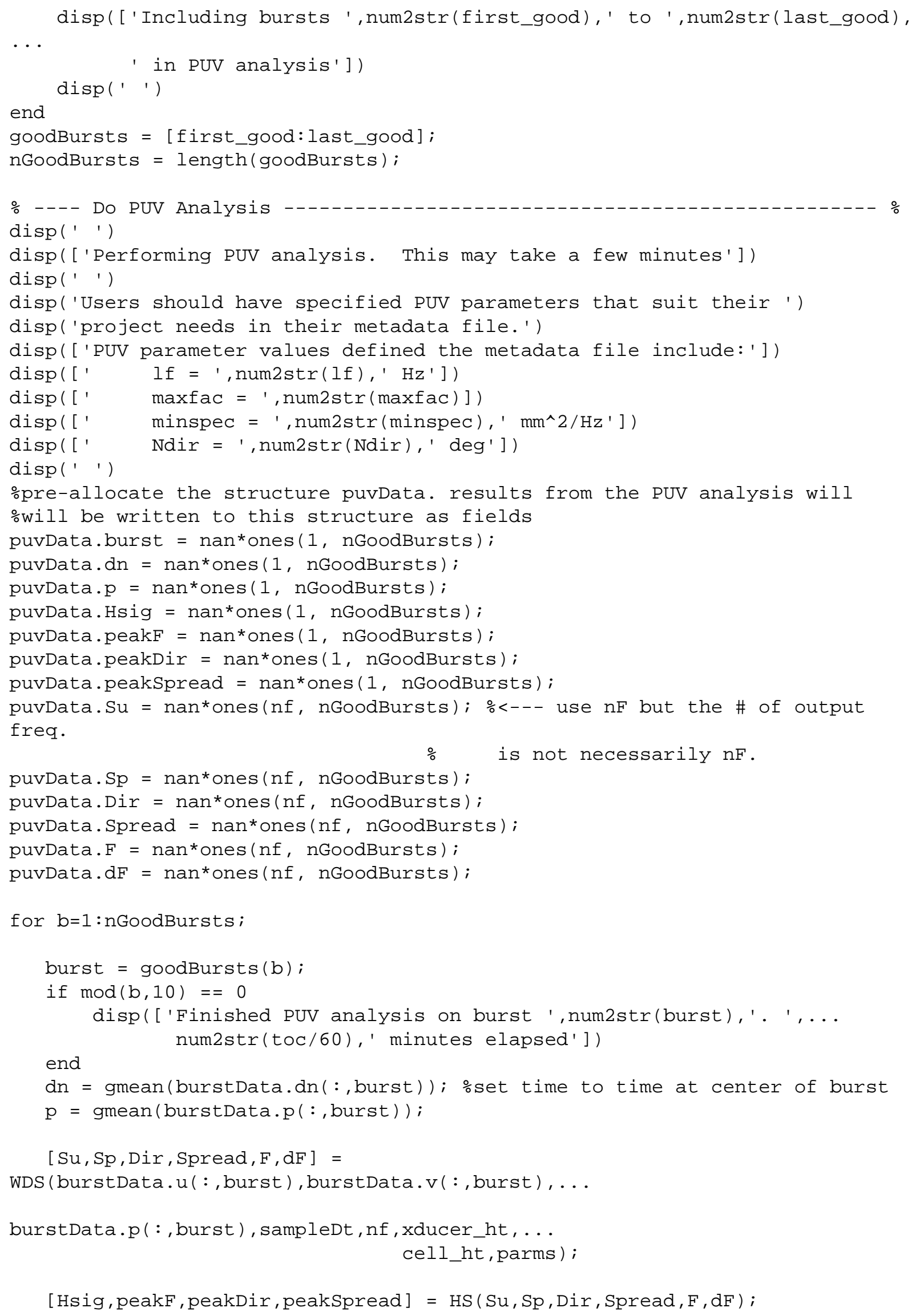




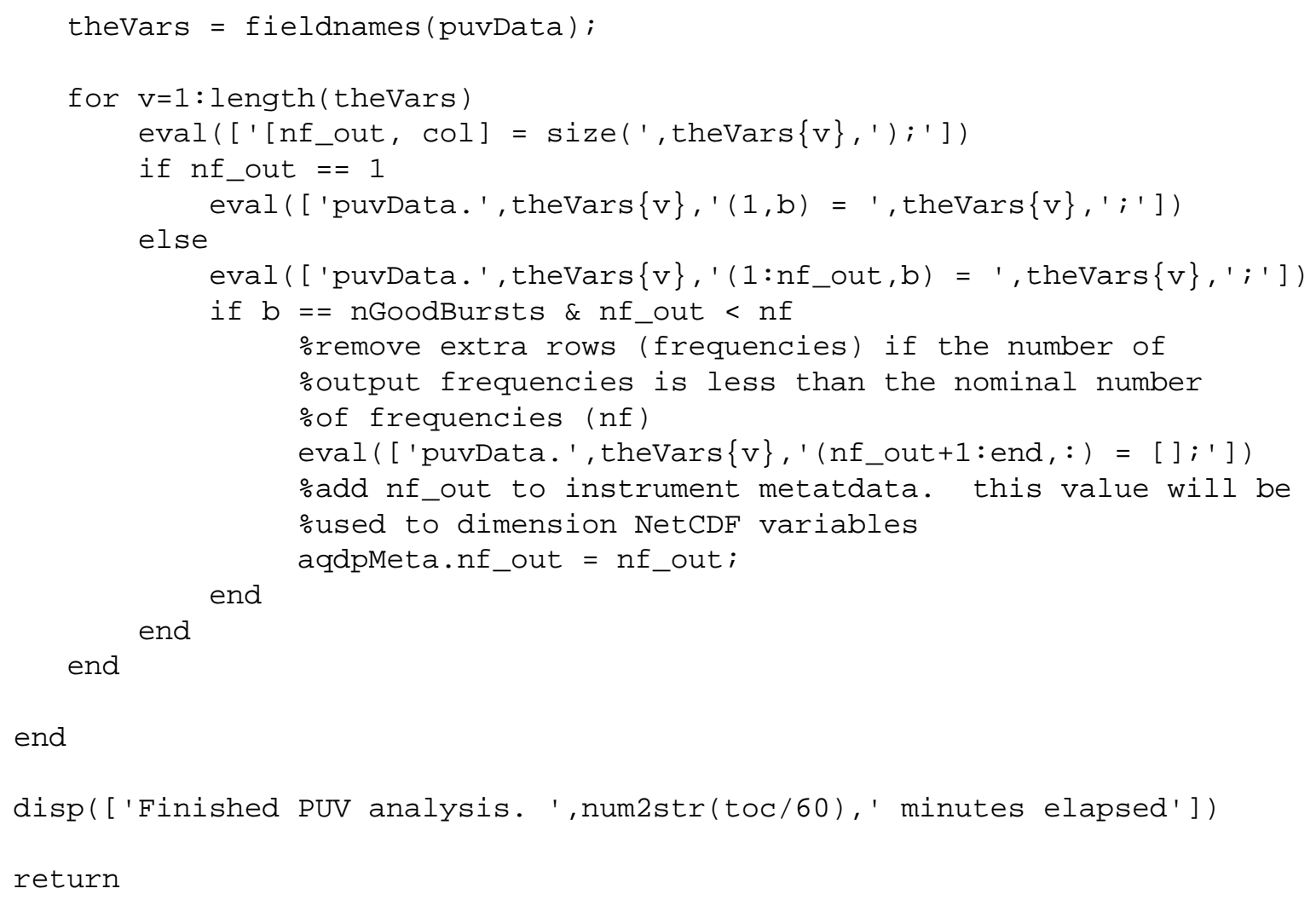




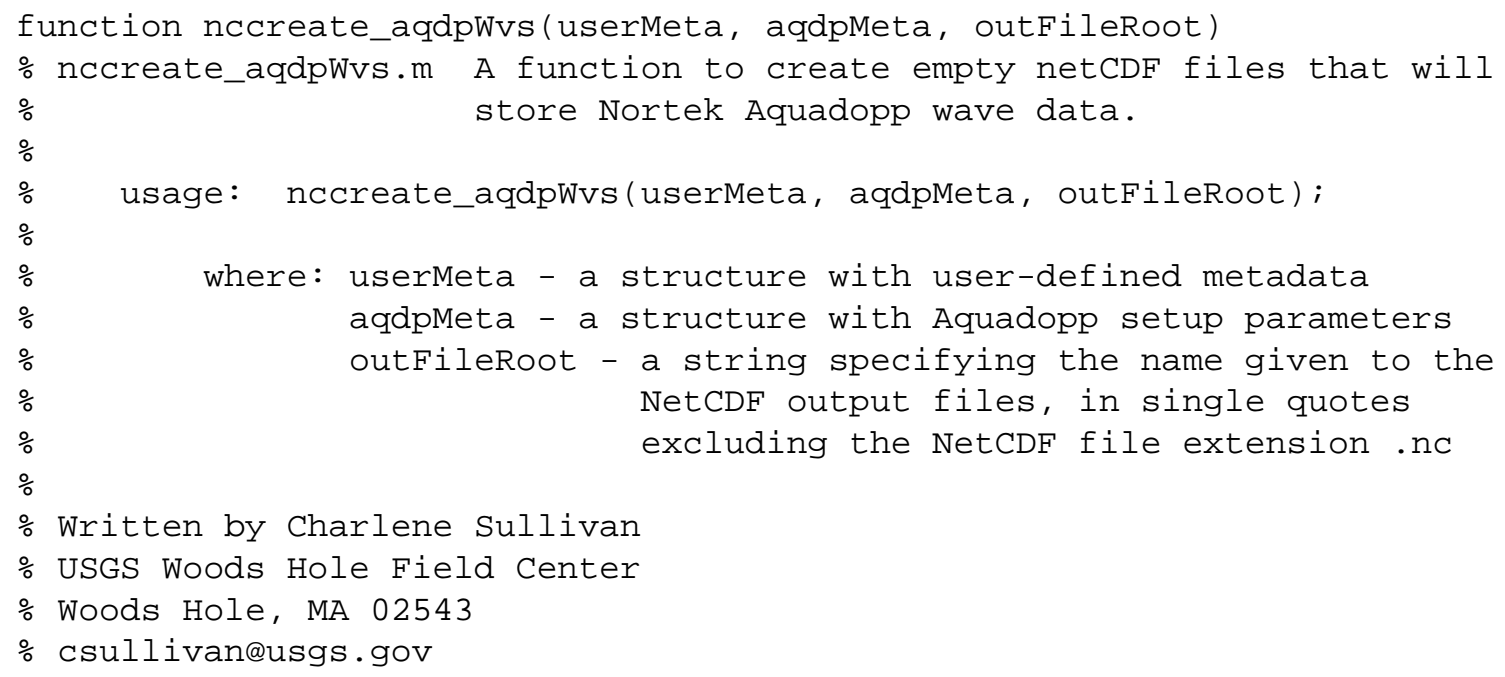

\% C. Sullivan $03 / 29 / 06$, version 1.3

$\%$ Add EPIC codes to the variables wp_peak and wvdir.

$\%$ C. Sullivan 10/28/05, version 1.2

$\%$ Provide the user additional feedback regarding code execution. Changed

$\%$ DATA_TYPE attribute description to be consistent $\mathrm{w} /$ the documentation.

\% Changed EPIC code and units on the variable lon to 502 and degree_east

$\%$ for consistency $w /$ longitude as specified in the metadata file where west

$\%$ is negative.

$\%$ C. Sullivan 06/09/05, version 1.1

$\%$ The variables 'pspec' and 'vspec' will now have units of mm/sqrt(Hz) for

$\%$ consistency with RDI and SonTek spectra. (the old units were $\mathrm{m}^{\wedge} 2 / \mathrm{Hz}$ )

$\%$ C. Sullivan 06/02/05, version 1.0

\% PUV analysis now run as part of the toolbox. Instrument setup information

$\%$ is now included in the metadata. Two NetCDF output files are created;

$\%$ one file has the raw pressure and velocity data that was used in PUV

$\%$ analysis; the other has the processed data from the PUV analysis.

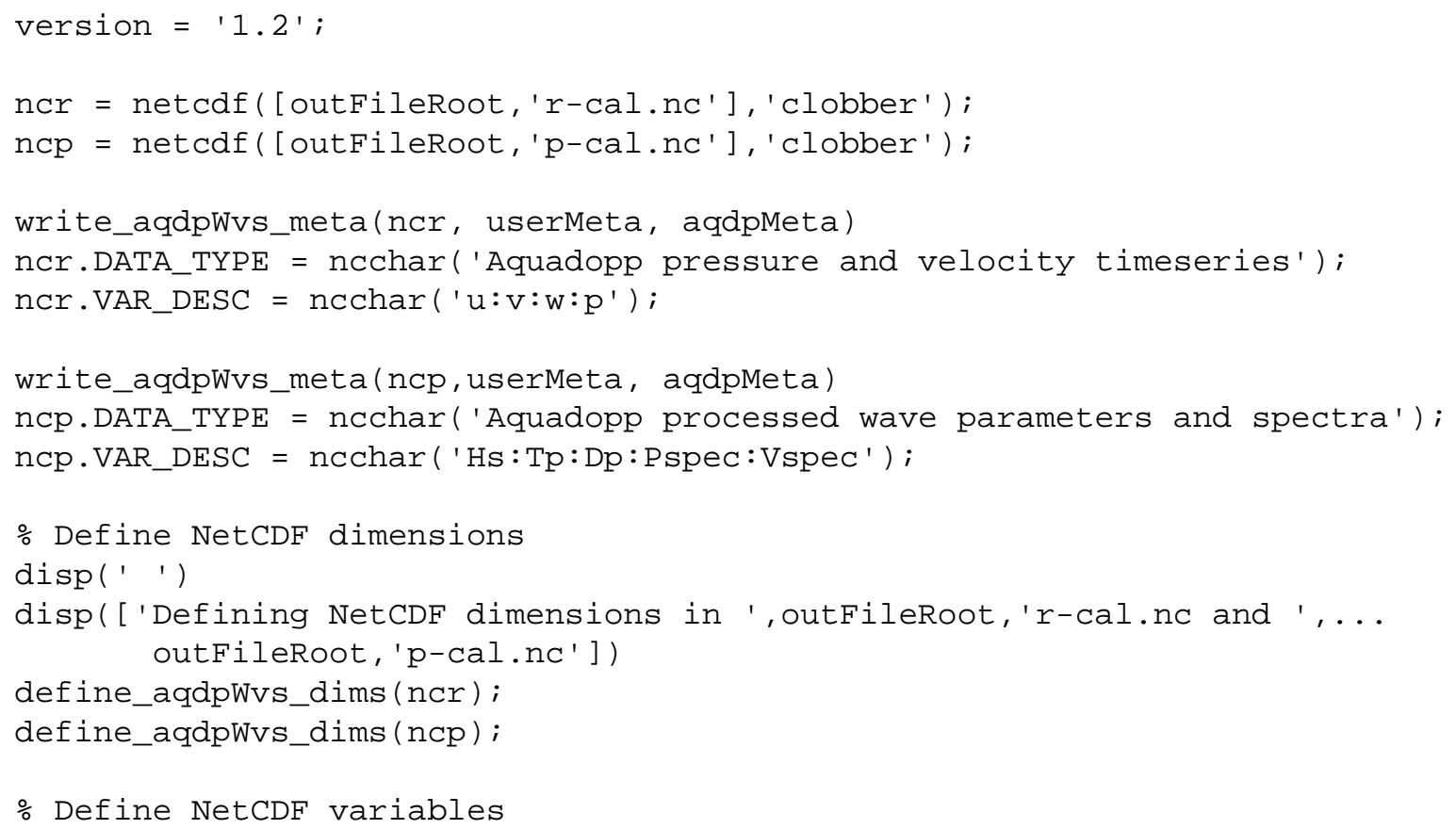


disp(['Defining NetCDF variables in ', outFileRoot, ' $r$-cal.nc and ', $\ldots$ $\operatorname{disp}\left({ }^{\prime}\right.$ ' $)$ outFileRoot, 'p-cal.nc'])

define_aqdpWvs_vars(ncr);

define_aqdpWvs_vars(ncp);

endef(ncr);

ncr $=$ close $($ ncr $)$;

endef(ncp);

ncp $=\operatorname{close}($ ncp $)$;

return

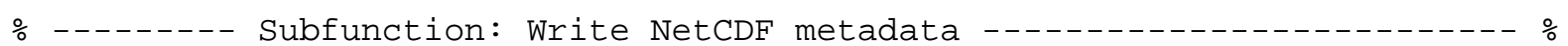
function write_aqdpWvs_meta(nc, userMeta, aqdpMeta)

theAtts = fieldnames (userMeta);

for $a=1$ : length (theAtts)

eval $([$ 'theDef $=$ userMeta. ', theAtts $\{a\}, ' ; '])$

if ischar(theDef)

else

eval(['nc.' ', theAtts $\{a\}, '=\operatorname{ncchar}\left({ }^{\prime} '\right.$ ', theDef, ' ' ' $\left.\left.) ; '\right]\right)$; end

$\operatorname{eval}([$ 'nc. ', theAtts $\{a\}, '=\operatorname{ncfloat}([$ theDef $]) ; '])$;

end

theAtts $=$ fieldnames $($ aqdpMeta $) ;$

for $a=1$ : length(theAtts)

eval $\left(\left[\right.\right.$ 'theDef $^{\prime}$ aqdpMeta. ', theAtts $\left.\left.\{a\}, ' ; '\right]\right)$

if ischar(thedef)

else

eval( ['nc.' , theAtts $\{a\}, '=\operatorname{ncchar}\left({ }^{\prime} '\right.$ ', thedef, ' ' ');']);

$\operatorname{eval}\left(\left[\right.\right.$ 'nc. $^{\prime}, \operatorname{theAtts}\{a\}, '=\operatorname{ncfloat}([$ theDef $\left.\left.]) ; '\right]\right) ;$

end end

return

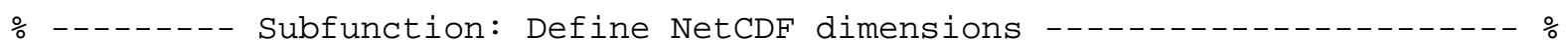
function define_aqdpWvs_dims(nc)

\%common dimensions for both raw and processed NetCDF files

nc $($ 'time') $=0$;

$\mathrm{nc}($ 'lat') $=1$;

$n c\left(\right.$ 'lon' $^{\prime}=1$;

if strcmp(nc.DATA_TYPE(:), 'Aquadopp processed wave parameters and spectra')

\%dimensions specific to processed NetCDF file

analysis

$\mathrm{nc}($ 'frequency') $=\mathrm{nc} . \mathrm{nf}$ _out $(:)$; \%this is a result from the PUV

elseif strcmp(nc.DATA_TYPE(:), 'Aquadopp pressure and velocity timeseries')

\%dimensions specific to raw NetCDF file 
end

nc $($ 'sample' $)=$ nc.Wave_Number_of_samples $(:)$;

return

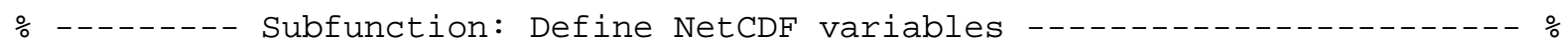

function define_aqdpWvs_vars(nc)

\%coordinate variables are the same for both the raw and

\%processed NetCDF files

nc $\{$ 'burst' $\}=$ nclong('time');

nc $\{$ 'burst' $\}$. FORTRAN_format $=$ ncchar ('F10.2');

nc $\{$ 'burst' $\}$. units $=$ ncchar ('counts');

nc $\{$ 'burst' $\}$.type = ncchar ('EVEN' $)$;

nc $\{$ 'lat' $\}=$ ncfloat ('lat');

nc $\{$ 'lat' $\}$.FORTRAN_format $=\operatorname{ncchar}($ 'F10.4');

nc $\{$ 'lat' $\}$.units $=$ ncchar ('degree_north');

nc $\{$ 'lat' $\}$.type $=$ ncchar ('EVEN');

nc $\{$ 'lat' $\}$.epic_code $=$ nclong $(500)$;

nc $\{$ 'lon' $\}=\operatorname{ncfloat}($ 'lon');

nc $\{$ 'lon' $\}$.FORTRAN_format $=$ ncchar ('F10.4');

nc $\{$ 'lon' $\}$.units $=$ ncchar ('degree_east');

nc $\{$ 'lon' $\}$.type $=$ ncchar ('EVEN');

nc $\{$ 'lon' $\}$.epic_code $=$ nclong $(5 \odot 2)$;

if strcmp(nc.DATA_TYPE(:), 'Aquadopp processed wave parameters and spectra')

\%Record variables for ONLY the processed data NetCDF file.

\%EPIC variables

nc $\{$ 'time' $\}=$ nclong('time');

nc $\{$ 'time' $\}$. FORTRAN_format $=$ ncchar $(' F 10.2 ')$;

nc $\{$ 'time' $\}$.units $=$ ncchar ('True Julian Day');

nc $\{$ 'time' $\}$. type $=$ ncchar ('EVEN');

nc $\{$ 'time' $\}$.epic_code $=$ nclong $(624)$;

nc $\{$ 'time2' $\}=$ nclong('time');

nc $\{$ 'time2' $\}$.FORTRAN_format $=$ ncchar $(' F 10.2 ')$;

nc $\{$ 'time2' $\}$. units $=$ ncchar $(' m s e c$ since $\odot: \odot \odot$ GMT');

nc $\{$ 'time2' $\}$.type $=$ ncchar ('EVEN');

nc $\{$ 'time2' $\}$.epic_code $=$ nclong $(624)$;

nc $\{$ 'wh_4061' $\}=$ ncdouble('time', 'lat', 'lon');

nc $\{$ 'wh_4061'\}.long_name=ncchar ('Significant Wave Height $(m)$ ');

nc $\{$ 'wh_4061' $\}$.generic_name=ncchar ( 'Hs') ;

nc $\{$ 'wh_4061' $\}$. units=ncchar (' $m$ ') ;

nc $\{$ 'wh_4061' $\}$.epic_code=nclong (4061);

nc $\{$ 'wh_4061' $\}$. FORTRAN_format=ncchar ( 'F10.2' );

nc $\{$ 'wh_4061' $\}$.Fillvalue $=1.0000000409184788 \mathrm{E} 35$;

nc $\{$ 'wh_4061' $\}$. minimum $=\operatorname{ncfloat}(\Theta)$;

nc $\{$ 'wh_4061' $\}$. maximum $=\operatorname{ncfloat}(\Theta)$;

nc $\{$ 'hght_18' $\}=$ ncdouble('time', 'lat', 'lon');

nc $\{$ 'hght_18'\}. long_name=ncchar ('Height of the Sea Surface $(m) ')$; 


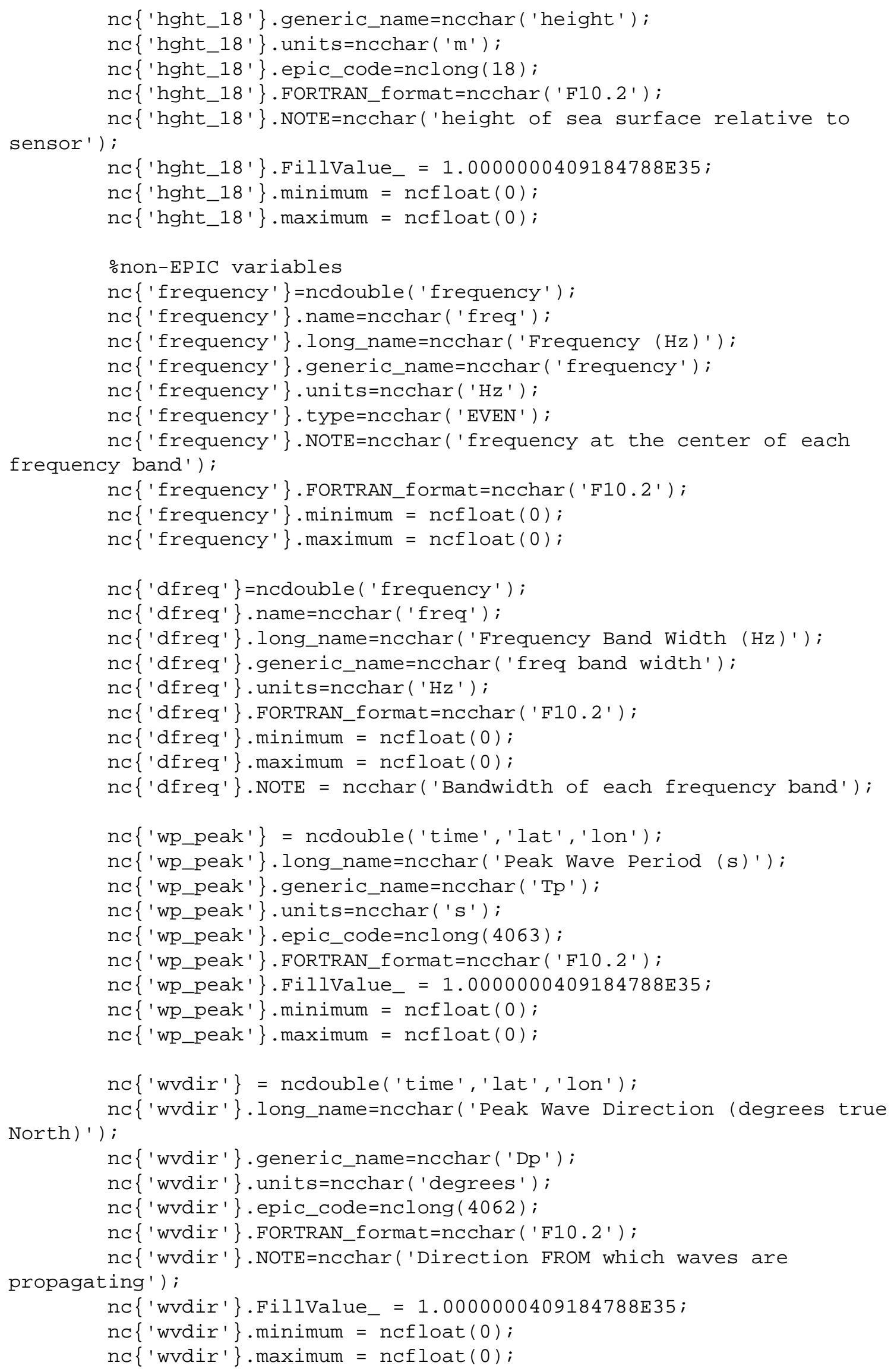




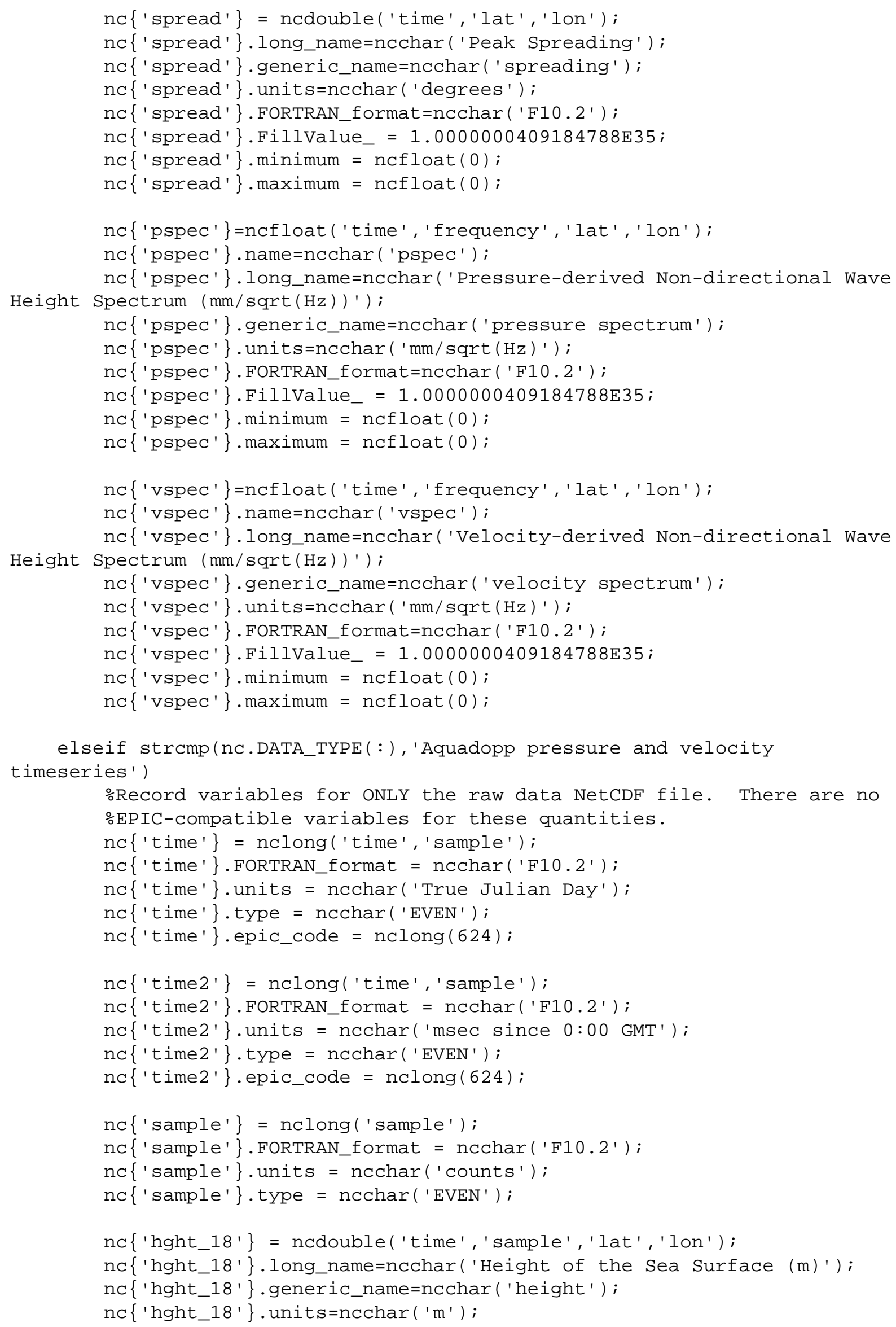




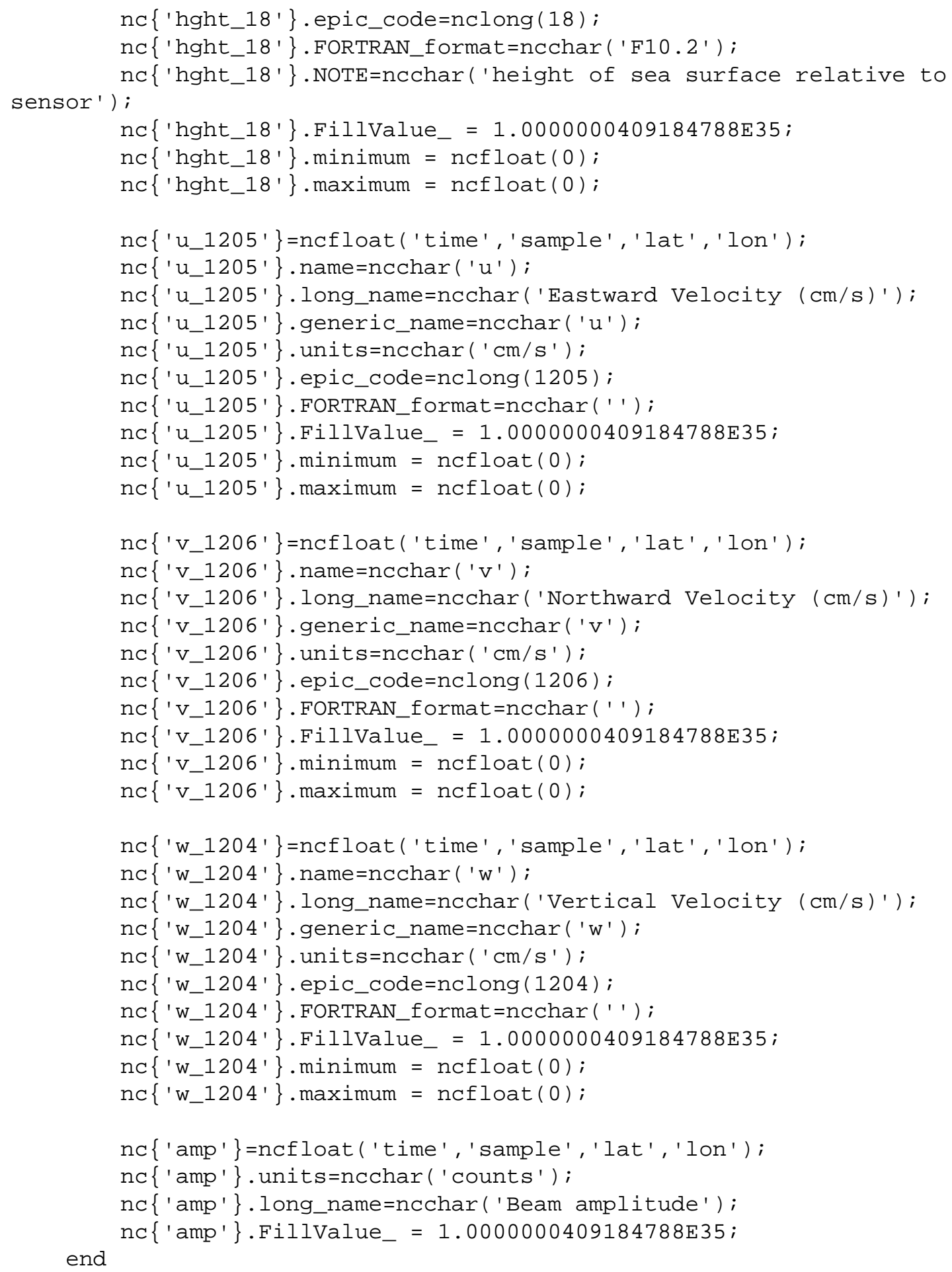

return 


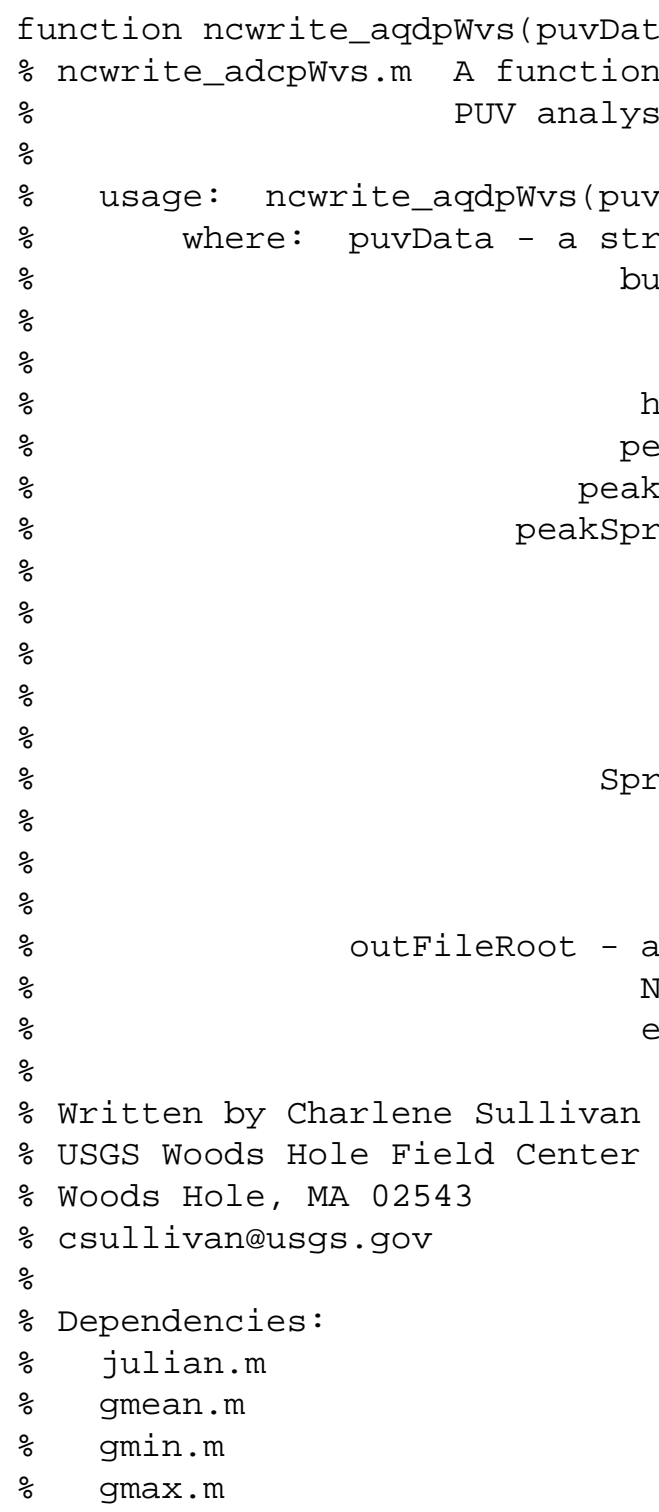

$\%$ C. Sullivan $03 / 29 / 06$, version 1.4

$\%$ Reverse the chronology on the history attribute so the most recent

$\%$ processing step is listed first. For spectra variables, calculate min

$\%$ and max attributes over time for each frequency.

$\%$ C. Sullivan 10/28/05, version 1.3

\% ADCP wave direction is direction FROM!! Wave direction from PUV analysis

$\%$ is direction TO, so convert it do direction FROM to be consistent with

$\%$ ADCP waves.

$\%$ C. Sullivan $06 / 13 / 05$, version 1.2

$\%$ Wave direction, 'wvdir', is output by PUV analysis as 'direction from'.

$\%$ Convert wave direction to 'direction to' for consistency with ADCP and

$\%$ Argonaut peak wave directions.

$\%$ C. Sullivan $06 / 09 / 05$, version 1.1

$\%$ The variables 'pspec' and 'vspec' are converted from $\mathrm{m}^{\wedge} 2 / \mathrm{Hz}$ to

$\% \mathrm{~mm} / \mathrm{sqrt}(\mathrm{Hz})$

$\%$ C. Sullivan $06 / 02 / 05$, version 1.0

$\%$ This function writes the data from PUV analysis to the processed data

$\%$ NetCDF file. 


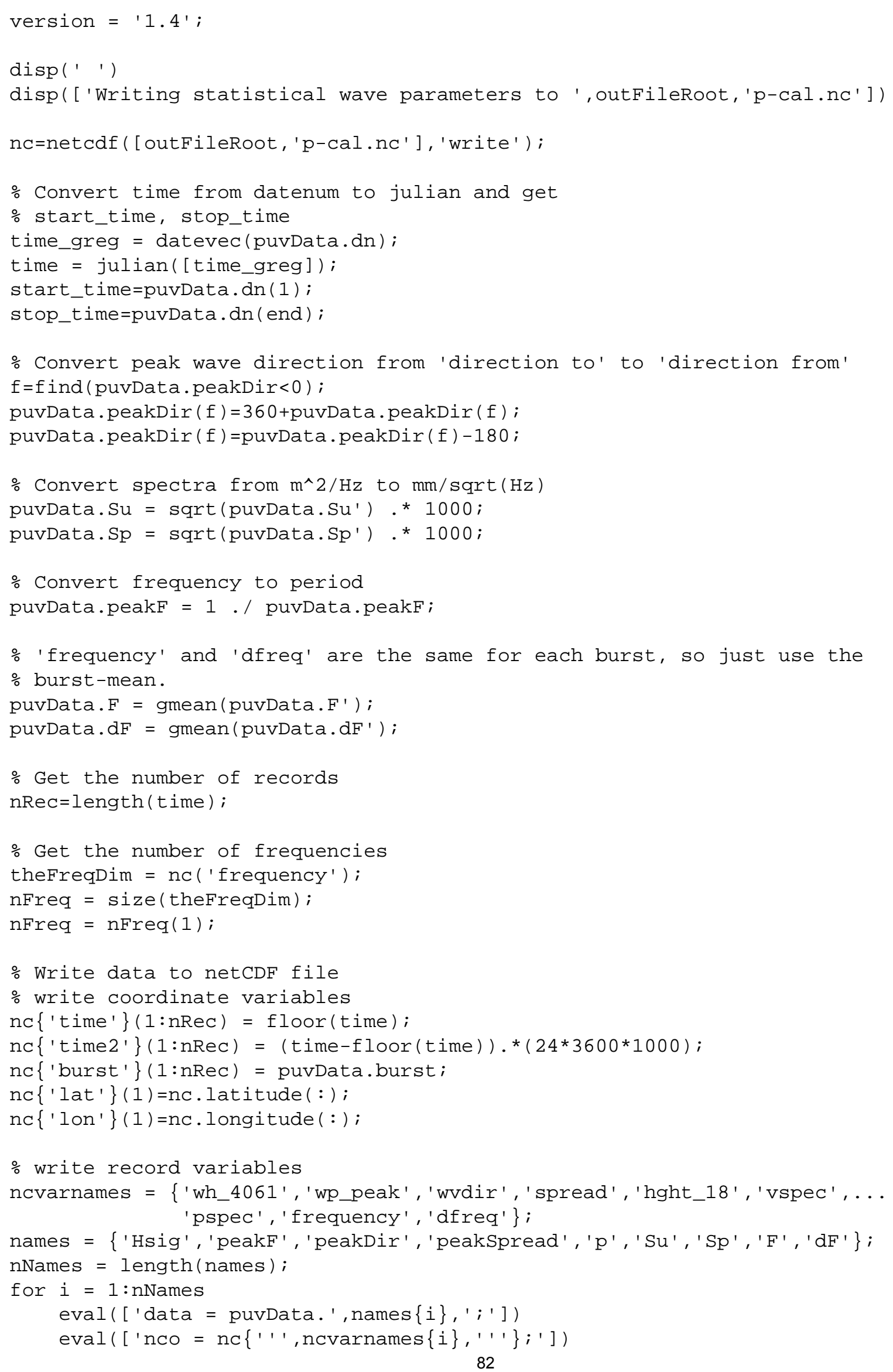




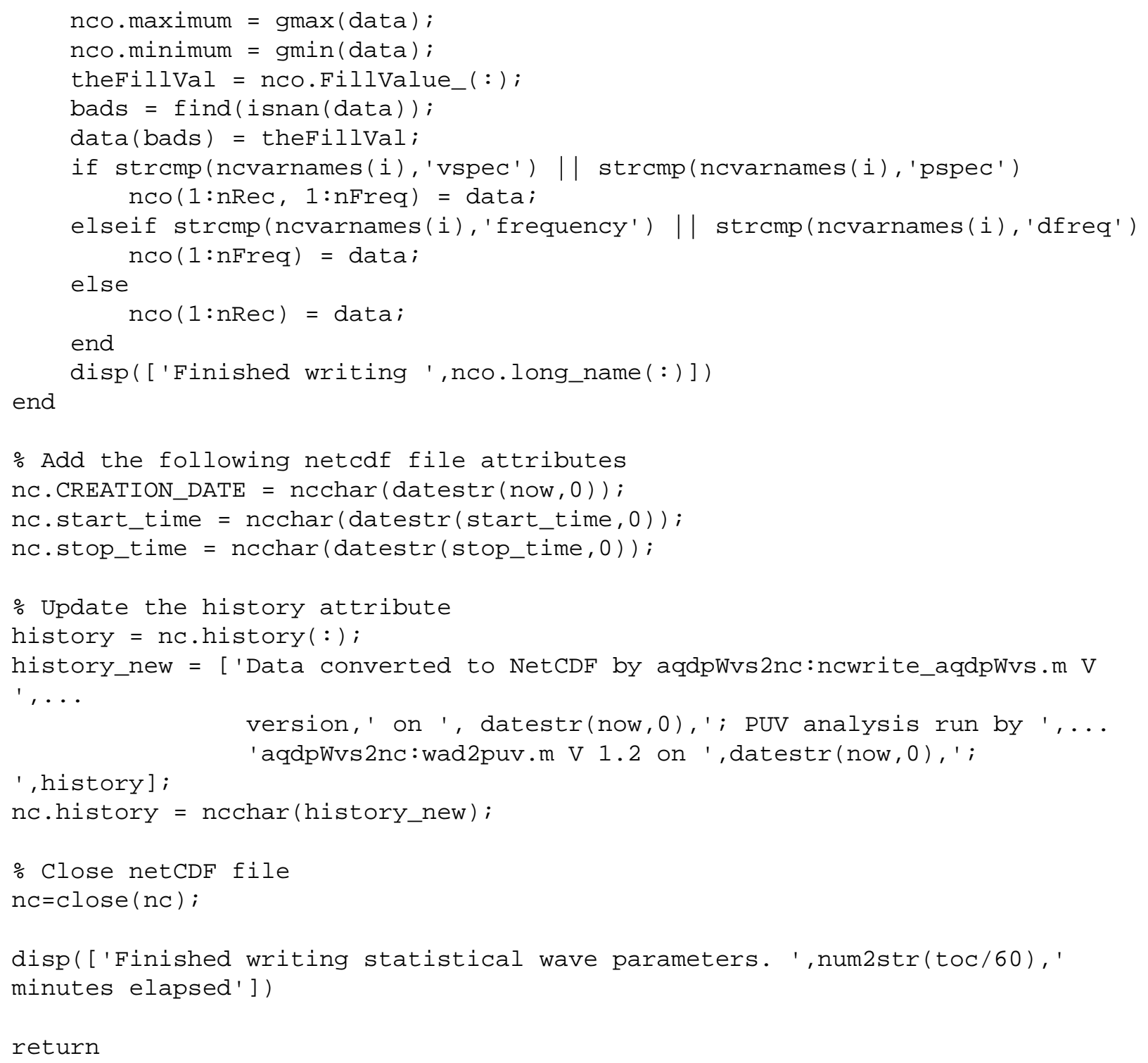




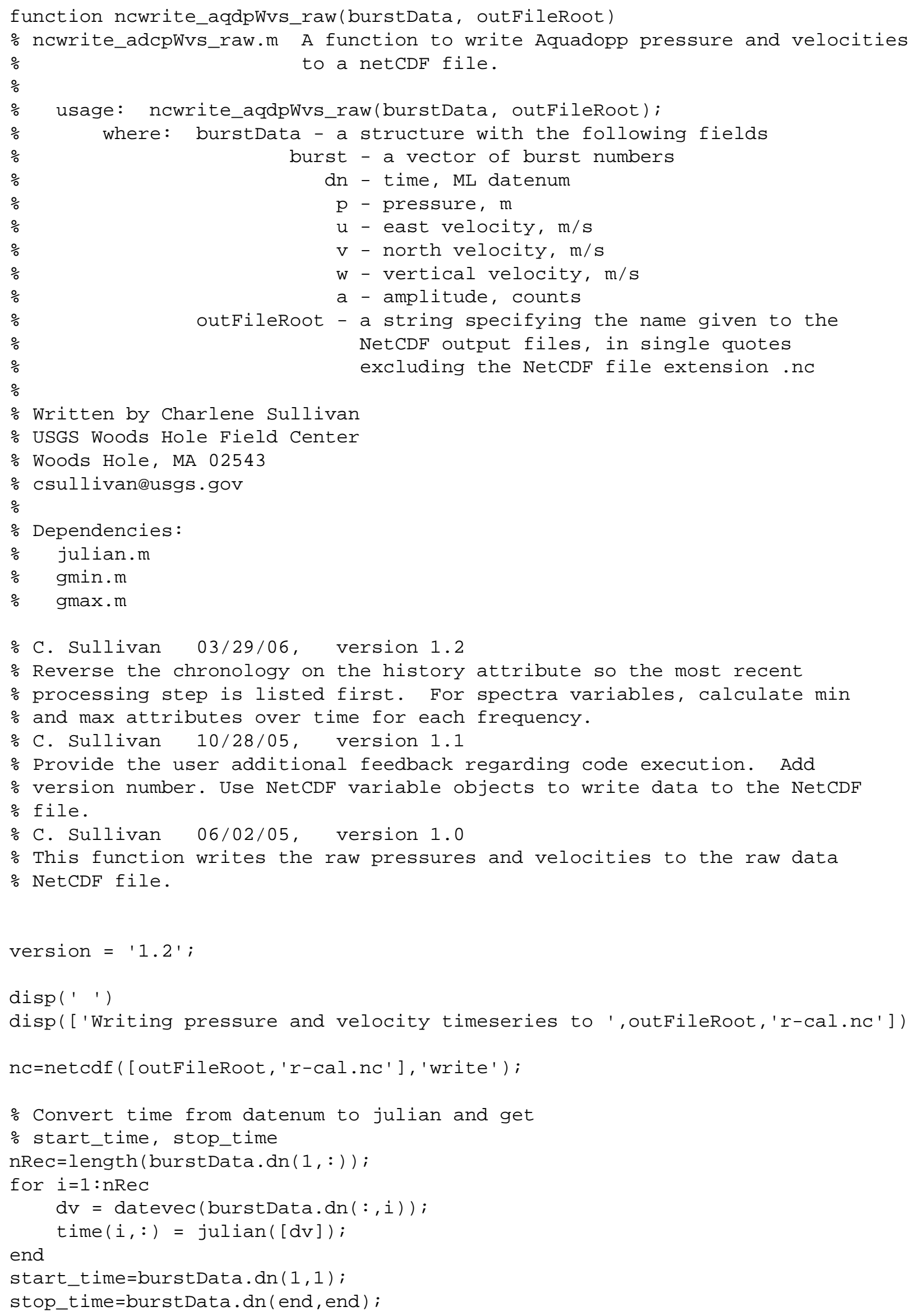


\% Convert velocities from $\mathrm{m} / \mathrm{s}$ to $\mathrm{cm} / \mathrm{s}$ for EPIC-compatibility

burstData.u = burstData.u $* 100$;

burstData.v = burstData.v* 100 ;

burstData.w = burstData.w*100;

$\%$ Get the number of records

$\mathrm{nRec}=\operatorname{size}($ time, 1$)$;

$\%$ Get the number of samples

theSampleDim = nc ('sample');

nSamples = size (thesampleDim);

nSamples $=$ nSamples $(1)$;

$\%$ Write data to netCDF file

$\%$ write coordinate variables

nc $\{$ 'time' $\}(1:$ nRec, $1:$ nSamples $)=$ floor $($ time $)$;

nc $\{$ 'time2' $\}(1:$ nRec, 1 :nSamples $)=($ time-floor (time $)) .{ }^{*}(24 * 3600 * 1000)$;

nc $\{$ 'burst' $\}(1: \mathrm{nRec})=$ burstData.burst;

nc $\{$ 'lat' $\}(1)=$ nc . latitude $(:)$;

nc $\{$ 'lon' $\}(1)=$ nc. longitude (: :);

nc $\{$ 'sample' $\}(1:$ nSamples $)=[1:$ nSamples $]$ ';

$\%$ write record variables

ncvarnames $=\{$ 'hght_18', 'amp ', 'u_1205', 'v_1206', 'w_1204' $\}$;

names $=\left\{{ }^{\prime} p^{\prime},{ }^{\prime} a^{\prime},{ }^{\prime} u^{\prime},{ }^{\prime} v^{\prime},{ }^{\prime} w^{\prime}\right\}$;

nNames $=$ length (names);

for $i=1:$ nNames

$\operatorname{eval}([$ 'data $=$ burstData. ', names $\{i\}, ' ; '])$

$\operatorname{eval}\left(\left[\right.\right.$ 'nco $=\operatorname{nc}\left\{{ }^{\prime} '\right.$ ', ncvarnames $\left.\left.\left.\{i\}, '{ }^{\prime} '\right\} ; '\right]\right)$

nco. $\operatorname{maximum}=\operatorname{gmax}($ data $)$;

nco.minimum $=$ gmin $($ data $) ;$

theFillval = nco. FillValue_(:);

bads = find $($ isnan $($ data $))$;

data $($ bads $)=$ theFillval;

nco(1:nRec, 1:nSamples $)=$ data;

end

disp(['Finished writing ',nco.long_name(:)])

$\%$ Add the following netcdf file attributes

nc. CREATION_DATE $=\operatorname{ncchar}(\operatorname{datestr}($ now, 0$))$;

nc.start_time $=$ ncchar $($ datestr $($ start_time, 0$))$;

nc.stop_time $=$ ncchar $(\operatorname{datestr}($ stop_time, 0$))$;

\% Update the history attribute

history = nc. history $(:)$;

history_new $=$ ['Pressure and velocity timeseries converted to NetCDF by ',$\ldots$ 'aqdpWvs2nc:ncwrite_aqdpWvs_raw.m $\vee$ ', version,' on ', ... datestr $($ now, 0$)$, ' ; ', history];

nc.history $=$ ncchar (history_new);

$\%$ Close netCDF file

$\mathrm{nc}=\mathrm{close}(\mathrm{nc})$;

disp(['Finished writing Aquadopp pressure and velocity timeseries. ', ... num2str(toc/60),' minutes elapsed']) 
return 